\title{
Computational anisotropic hardening multiplicative elastoplasticity based on the corrector elastic logarithmic strain rate
}

\author{
Miguel Á. Sanz, Francisco J. Montáns, Marcos Latorre* \\ Escuela Técnica Superior de Ingeniería Aeronáutica y del Espacio, Universidad Politécnica de Madrid, Plaza Cardenal Cisneros, 3 , \\ 28040-Madrid, Spain
}

Received 29 September 2016; received in revised form 21 January 2017; accepted 20 February 2017

Available online 27 March 2017

\section{Highlights}

- New computational framework for multiplicative anisotropic elastoplasticity.

- Based on a six-dimensional additive corrector update for elastic logarithmic strains.

- No hypothesis needed for the plastic spin in order to integrate the symmetric flow.

- Yields a fully symmetric finite element formulation parallel to the infinitesimal one.

- Overcomes the so-called "rate issue" from a computational standpoint.

\begin{abstract}
In this paper we present a new computational framework for anisotropic elastoplasticity with mixed hardening which presents the following characteristics: (1) it is motivated by a one-dimensional rheological model where the main differences are due to geometric nonlinearities and three-dimensional effects; (2) it uses the Lee multiplicative decomposition; (3) it is valid for anisotropic yield functions; (4) it is valid for any anisotropic stored energy, either linear or nonlinear in logarithmic strains; (5) it is valid for (non-moderate) large elastic strains; (6) it results in a six-dimensional additive corrector update, parallel to that of the infinitesimal theory; (7) it does not explicitly employ plastic strain tensors or plastic metrics, circumventing definitely the "rate issue"; (8) the incremental plastic flow is isochoric using a simple backward-Euler scheme, without explicitly using exponential mappings; (9) no hypothesis is needed for the plastic spin in order to integrate the symmetric flow derived from the dissipation equation; (10) the Mandel stress tensor plays no role in the formulation; (11) it yields a fully symmetric algorithmic linearization consistent with its associative nature and the principle of maximum dissipation; and (12) it recovers the formulation of Simó for isotropy as a particular case.
\end{abstract}

(C) 2017 Elsevier B.V. All rights reserved. 


\section{Introduction}

The development of anisotropic elastoplastic material models and algorithms for finite element implementation plays a fundamental role in industrial processes [1-4]. In manufacturing processes of metals, the influence of elastic anisotropy is important, for example during elastic recovery processes as the springback. The consideration of large elastic strains in these models and algorithms becomes also important for the numerical analysis of some polymers, which undergo large elastic strains accompanied by molecular chain reorientations, developing a high elastic anisotropy.

Constitutive models and integration algorithms for infinitesimal elastoplasticity, relying mainly on the classical return mapping schemes of Wilkins [5], Maenchen and Sacks [6] and Krieg and Key [7], have achieved nowadays an acceptable accuracy and efficiency [2,8,9]. Much effort has been done in order to extend the small strain formulations to the finite deformation regime [10], always with the main aim of preserving as much as possible the simplicity of the infinitesimal formulation through an algorithm that computes the closest point projection of the trial stresses onto the elastic domain [11]. Very different extended formulations, restricted to isotropy or including some kind of anisotropy, can be found in the literature, as for example computational models based on hypoelastic relations for stresses in rate form [12-17]; models formulated in terms of different types of stress and strain measures [18-23,11]; models employing different internal variables as the basic ones [24-27]; and hyperelastic formulations based on either plastic metrics [28-34] or the multiplicative decomposition of the deformation gradient [35-45]. Unlike additive plasticity models, multiplicative plasticity models are micromechanically motivated from single crystal metal plasticity [46,47], with the elastic part of the deformation gradient accounting for the elastic lattice deformation, so the corresponding strain energy may be considered well defined. A recent publication considering the numerics of the multiplicative decomposition, including combined isotropic-kinematic hardening models, can be found in [48]. However, the most controversial aspect of the theory is arguably associated with the derivation of continuum evolution equations for the plastic flow [49] and with their numerical integration [50], i.e. the "rate issue" as coined by Simó [11].

Regarding this last issue, the formulation and further integration of evolution equations expressed in terms of the plastic deformation gradient rate tensor, which goes back to the works of Eterovic and Bathe [21] and Weber and Anand [20], is probably the most common approach. A different approach relies on the ideas of the early works of Eckart [24], Besseling [25] and Leonov [26], who suggested that the internal elastic strains, from which the stresses directly derive, should govern the internal dissipation [27]. Indeed, from a strictly numerical viewpoint, this is the key feature of the classical integration algorithms [5-7], where the dissipative return onto the elastic domain is governed by the trial elastic stresses. Certainly, the extension of these ideas to the finite strain anisotropic case is not straightforward. Following this line, Simó and Miehe derived a continuum flow rule expressed in terms of the Lie derivative of the elastic left Cauchy-Green deformation tensor [51], which was further integrated in terms of elastic logarithmic strains yielding a computational framework that preserved the main structure of the classical return mapping schemes [11]. However, the formulation is fully restricted to isotropy, so an extrapolation to the anisotropic context that preserves the appealing structure of the infinitesimal plasticity algorithms without modification is missing.

In Ref. [49] we present a new class of flow rules valid for anisotropic elastoplasticity and large elastic strains consistent with the Lee multiplicative decomposition. Generalizing Simó's approach [11], internal elastic strain variables are taken as the basic variables, so the evolution equations become entirely formulated in terms of corrector elastic strain rates rather than plastic ones. The concepts of variable dependencies, partial differentiation and mapping tensors [52] play a fundamental role on the kinematics. The procedure is described in different configurations and in terms of different stress and strain measures. Importantly, the generally non-symmetric Mandel stress tensor [53], power-conjugate of the plastic velocity gradient in the intermediate configuration, is not present in the dissipation inequality if one follows this approach. On the contrary, respective thermodynamical symmetric stress tensors and power-conjugate elastic symmetric strain rates govern the dissipation process in the selected configuration. In each case, an associative flow rule based on the notion of the corrector elastic strain rate is trivially obtained consistently with the principle of maximum dissipation. These flow rules may be considered conventional in the sense that they adopt the same structure as the small strain one, hence being simplified to a great extent and definitively solving the "rate issue" in its continuum part. The particularization of the spatial formulation to isotropy and with the additional restriction of vanishing plastic spin [54] gives as a result the Simó framework. Another interesting characteristic of the six-dimensional flow rules derived in Ref. [49] is that the plastic spin does not take explicit part in them, exactly as it occurs in the infinitesimal theory. Consistently with Ref. [55], any flow rule for the plastic spin must be postulated as an additional hypothesis independent of the maximum dissipation principle. 
In this work, we present a continuum elastoplasticity framework and implicit computational formulation in full space description valid for anisotropic stored energies and large elastic strains consistent with the Lee multiplicative decomposition. As a fundamental ingredient, we take special advantage of the flow rule given in Ref. [49] written in terms of the corrector elastic logarithmic symmetric strain rate tensor [56] and its power-conjugated generalized Kirchhoff symmetric stress tensor, both defined in the intermediate configuration. Importantly, this flow rule may be immediately recast in a remarkably simple incremental form by direct backward-Euler integration, resulting into an additive return mapping scheme in full tensorial form that preserves the appealing structure of the classical schemes of infinitesimal plasticity without modification, hence solving the "rate issue" in its computational part as well. Exact preservation of plastic volume for pressure insensitive yield criteria is readily accomplished in this case. Since the formulation is entirely written in terms of the elastic logarithmic strains in the intermediate configuration, the plastic deformation gradient tensor is updated in a proper incremental fashion at each converged step as an additional independent assumption inherently related with the skew-symmetric flow. As we show, our associative flow rule written directly in terms of the elastic logarithmic strain evolution yields a fully symmetric finite element formulation of finite strain anisotropic multiplicative elastoplasticity, thus generalizing the solution restricted to isotropy that Simó and Miehe [51] provided for an open issue raised in the pioneering work of Argyris et al. [57]. The Simo's integration scheme in principal strain/stress directions is recovered (in its unrotated form) when the formulation is restricted to isotropy, even though the exponential mapping is not explicitly employed.

We include kinematic hardening effects in the present model following a novel, different approach to the standard methodology based on the explicit consideration of a backstress tensor. We motivate the kinematic hardening formulation from a one-dimensional "think" (rheological) model [58]. The macroscopic backstress may thereafter be implicitly determined as a result, if desired. Furthermore, this formulation, which has some similarities with others in the literature based on the Armstrong-Frederick kinematic hardening, e.g. [59], which is based on the pioneering work of Lion [37], enables us to use a very similar computational framework to the one we presented in Ref. [60] in the context of anisotropic finite strain viscoelasticity based on the Sidoroff multiplicative decomposition. Indeed, the formulation derived herein is equivalent in many aspects to that in Ref. [60]. This approach reproduces the observed kinematic hardening behavior from a macroscopic standpoint, even though without modifying the evolution equation and the stress integration algorithm.

The rest of the paper is organized as follows. We next present in Section 2 the ideas for one-dimensional infinitesimal elastoplasticity including combined hardening effects in both continuum and incremental forms. We present in Section 3 the large strain formulation in the intermediate configuration using directly logarithmic strains. Section 4 is devoted to the consistent linearization of the continuum elastoplastic response, where its similarity with the infinitesimal case can be noticed. In Section 5 we perform the integration of the flow rule and derive the implicit algorithmic formulation, showing that a fully symmetric consistent algorithmic tangent tensor is obtained parallel to the infinitesimal one. In Section 6 we explain how to determine the internal model parameters from experimental testing. Finally, in Section 7 some examples including homogeneous and non-homogeneous deformations and large elastic strains prove the consistency of the formulation and its excellent numerical efficiency.

The general formulation that we present (until Section 6), with nonlinear functions, presents a natural framework for anisotropic elastoplasticity of both metals, with moderately large elastic strains, and soft materials, with large elastic strains. However, the kinematic hardening laws used in the examples, linear in terms of logarithmic strains, may not be suitable for certain metals, for which the Armstrong-Frederick hardening is more appropriate.

\section{Motivation: infinitesimal elastoplasticity}

The purpose of this section is to motivate the model in the simpler infinitesimal one-dimensional description, within both the continuum and the algorithmic frameworks. The presented concepts result in a remarkable parallelism with the large strain algorithmic formulation presented in next sections.

\subsection{Continuum theory}

Consider the elastoplastic standard solid for small strains including a Prandtl (friction-spring) element in parallel with a single Hooke (spring) element shown in Fig. 1, where $\varepsilon$ and $\sigma$ are the external, measurable strain and stress, respectively, and $\varepsilon_{e}$ and $\varepsilon_{p}$ are internal, generally non-measurable variables describing the internal elastic and plastic behaviors. We will see below that the branch with the single spring element in the device of Fig. 1 is responsible for 


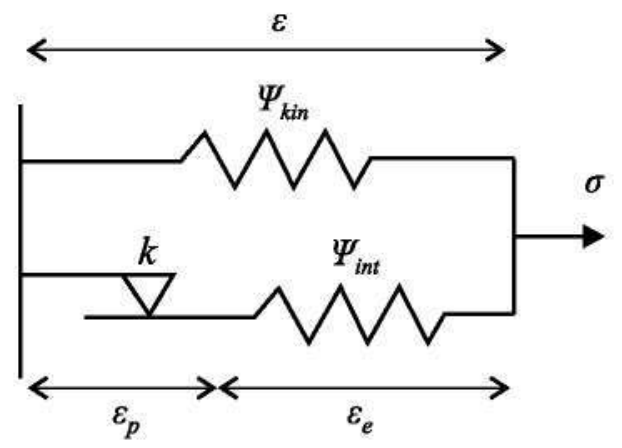

Fig. 1. Rheological model motivating the elastoplasticity model with combined isotropic and kinematic non-linear hardening.

the phenomenologically observable (say macroscopic) kinematic hardening of the model, hence we use the subscript $k i n$ in the strain energy function associated with that spring. We refer to the internal spring within the Prandtl branch in Fig. 1 with the subscript int. We denote the internal flow stress associated with the friction element as $k$. Note that the current approach to the kinematic hardening differs from the classical Prager-type kinematic hardening usually adopted for metals [61], which includes the Armstrong-Frederick hardening. In particular, even though the implemented approach in the examples below does not allow for stress saturation, stress saturation could be modeled by the present model using an appropriate nonlinear function $\Psi_{k i n}$.

We note that the elastoplastic rheological model shown in Fig. 1 presents an arrangement homologous to that of the viscoelastic one used in Ref. [60], where we have replaced the Newton (dashpot) element by a Saint-Venant (friction) element. This standard solid including kinematical hardening effects is also employed in, for example, Ref. [58] as a one-dimensional rheological model (therein named "think" model), where it is additionally compared to other more classical arrangements.

The internal strains in Fig. 1 relate to the external one through $\varepsilon=\varepsilon_{e}+\varepsilon_{p}$, so if we know the total deformation and one internal variable, then the another one is uniquely determined. We will consider $\varepsilon$ and $\varepsilon_{p}$ as the independent variables of the dissipative system and $\varepsilon_{e}$ will be the two-variable dependent internal variable, i.e.

$$
\varepsilon_{e}\left(\varepsilon, \varepsilon_{p}\right)=\varepsilon-\varepsilon_{p}
$$

which provides also a relation between the corresponding partial contributions to $\dot{\varepsilon}_{e}$ - we use the notation $\partial(\cdot) / \partial(0)$ for partial differentiation

$$
\dot{\varepsilon}_{e}=\left.\frac{\partial \varepsilon_{e}}{\partial \varepsilon}\right|_{\dot{\varepsilon}_{p}=0} \dot{\varepsilon}+\left.\frac{\partial \varepsilon}{\partial \varepsilon_{p}}\right|_{\dot{\varepsilon}=0} \dot{\varepsilon}_{p}=\dot{\varepsilon}-\dot{\varepsilon}_{p}=\left.\dot{\varepsilon}_{e}\right|_{\dot{\varepsilon}_{p}=0}+\left.\dot{\varepsilon}_{e}\right|_{\dot{\varepsilon}=0} .
$$

The stored energy in the device of Fig. 1 is given by $\Psi\left(\varepsilon, \varepsilon_{e}\right)=\Psi_{k i n}(\varepsilon)+\Psi_{i n t}\left(\varepsilon_{e}\right)$. The (non-negative) dissipation rate $\mathcal{D}$ is calculated from the external stress power $\mathcal{P}$ and the total strain energy rate $\dot{\Psi}$ through

$$
\mathcal{D}=\mathcal{P}-\dot{\Psi}_{k i n}-\dot{\Psi}_{i n t}=\sigma \dot{\varepsilon}-\sigma_{k i n} \dot{\varepsilon}-\sigma_{i n t}^{l e} \dot{\varepsilon}_{e} \geq 0
$$

where we define - we use the notation $d(\cdot) / d(0)$ for total differentiation

$$
\sigma_{k i n}:=\frac{d \Psi_{k i n}(\varepsilon)}{d \varepsilon} \quad \text { and } \quad \sigma_{i n t}^{\mid e}:=\frac{d \Psi_{i n t}\left(\varepsilon_{e}\right)}{d \varepsilon_{e}} .
$$

No dissipation takes place if we consider an isolated evolution of the external, independent variable $\dot{\varepsilon} \neq 0$, i.e. with $\dot{\varepsilon}_{p}=0$. Then $\left.\dot{\varepsilon}_{e} \equiv \dot{\varepsilon}_{e}\right|_{\dot{\varepsilon}_{p}=0}$ and Eq. (3) reads

$$
\mathcal{D}=\sigma \dot{\varepsilon}-\sigma_{k i n} \dot{\varepsilon}-\left.\sigma_{i n t}^{\mid e} \dot{\varepsilon}_{e}\right|_{\dot{\varepsilon}_{p}=0}=\left(\sigma-\sigma_{k i n}-\left.\sigma_{i n t}^{\mid e} \frac{\partial \varepsilon_{e}}{\partial \varepsilon}\right|_{\dot{\varepsilon}_{p}=0}\right) \dot{\varepsilon}=0 \quad \text { if } \quad \dot{\varepsilon}_{p}=0
$$

which yields

$$
\sigma=\sigma_{k i n}+\left.\sigma_{i n t}^{\mid e} \frac{\partial \varepsilon_{e}}{\partial \varepsilon}\right|_{\dot{s}_{p}=0}=\sigma_{k i n}+\sigma_{i n t}^{\mid e}=\sigma_{k i n}+\sigma_{i n t}
$$


where we recognize the following definition based on a chain rule operation - note the abuse of notation $\Psi_{i n t}\left(\varepsilon_{e}\right)=$ $\Psi_{i n t}\left(\varepsilon_{e}\left(\varepsilon, \varepsilon_{p}\right)\right)=\Psi_{i n t}\left(\varepsilon, \varepsilon_{p}\right)$

$$
\sigma_{i n t}:=\left.\sigma_{i n t}^{\mid e} \frac{\partial \varepsilon_{e}}{\partial \varepsilon}\right|_{\dot{\varepsilon}_{p}=0}=\frac{d \Psi_{i n t}\left(\varepsilon_{e}\right)}{d \varepsilon_{e}} \frac{\partial \varepsilon_{e}\left(\varepsilon, \varepsilon_{p}\right)}{\partial \varepsilon}=\frac{\partial \Psi_{i n t}\left(\varepsilon, \varepsilon_{p}\right)}{\partial \varepsilon}=\left.\frac{\partial \Psi_{i n t}}{\partial \varepsilon}\right|_{\dot{\varepsilon}_{p}=0} .
$$

Definitions of this type, based on the concept of partial differentiation, prove extremely useful in the finite deformation context, where they furnish the proper pull-back and push-forward operations between the different configurations being defined $[60,62,49]$.

Consider now an isolated internal evolution of the other independent variable in the problem, i.e. the case for which $\dot{\varepsilon}=0$ and $\dot{\varepsilon}_{p} \neq 0$. Then $\left.\dot{\varepsilon}_{e} \equiv \dot{\varepsilon}_{e}\right|_{\dot{\varepsilon}=0}$. The dissipation inequality of Eq. (3) must be positive because plastic deformation is taking place

$$
\mathcal{D}=-\left.\sigma_{i n t}^{\mid e} \dot{\varepsilon}_{e}\right|_{\dot{\varepsilon}=0}>0 \quad \text { if } \quad \dot{\varepsilon}_{p} \neq 0 .
$$

Since $\left.\dot{\varepsilon}_{e}\right|_{\dot{\varepsilon}=0}=-\dot{\varepsilon}_{p}$ and $\sigma_{i n t}^{\mid e}=\sigma_{i n t}$, recall Eqs. (2) and (6), then Eq. (8) adopts the usual form

$$
\mathcal{D}=\sigma_{i n t} \dot{\varepsilon}_{p}>0 \text { if } \dot{\varepsilon}_{p} \neq 0 \text {. }
$$

Eqs. (8) and (9) represent both the same physical concept, the former written in terms of the partial contribution $\left.\dot{\varepsilon}_{e}\right|_{\dot{\varepsilon}=0}$ to the total rate of the dependent internal variable $\varepsilon_{e}\left(\varepsilon, \varepsilon_{p}\right)$ and the latter written in terms of the total rate $\dot{\varepsilon}_{p}$ of the independent internal variable $\varepsilon_{p}$.

Inequality (8) is automatically fulfilled if we choose

$$
-\left.\dot{\varepsilon}_{e}\right|_{\dot{\varepsilon}=0}=\dot{\gamma} \frac{\sigma_{i n t}^{\mid e}}{k}
$$

which yields

$$
\mathcal{D}=\left(\frac{\sigma_{i n t}^{\mid e}}{k}\right)^{2} k \dot{\gamma}>0 \quad \text { if } \quad \dot{\varepsilon}_{p} \neq 0
$$

where $k>0$ is the yield stress of the internal frictional element of Fig. 1 and we define $\dot{\gamma} \geq 0$ as its power-conjugate plastic strain rate, as we see just below. If $k=k(\gamma)$ increases with the accumulated plastic deformation $\gamma=\int_{0}^{t} \dot{\gamma} d t$ we will obtain the case with combined kinematic and isotropic hardening. If $k$ is constant, the model describes the case with kinematic hardening only. We rephrase the dissipation Inequality (11) as

$$
\mathcal{D}=\left[\left(\frac{\sigma_{i n t}^{\mid e}}{k}\right)^{2}-1\right] k \dot{\gamma}+k \dot{\gamma}>0 \quad \text { if } \quad \dot{\gamma}>0 .
$$

If we force the plastic dissipation of the model to be given by the flow stress times the frictional strain rate - cf. Eqs, (8) and (9)

$$
\mathcal{D}=k \dot{\gamma} \geq 0 \quad \text { for } \quad \dot{\gamma} \geq 0
$$

then we immediately recognize the yield function and the loading/unloading Kuhn-Tucker conditions in Eq. (12), i.e.

$$
\dot{\gamma}>0 \Rightarrow f\left(\sigma_{i n t}^{\mid e}, k\right)=\left(\sigma_{i n t}^{\mid e}\right)^{2}-k^{2}=0 \quad \text { (plastic loading) }
$$

and

$$
f\left(\sigma_{i n t}^{\mid e}, k\right)=\left(\sigma_{i n t}^{\mid e}\right)^{2}-k^{2}<0 \Rightarrow \dot{\gamma}=0 \quad \text { (elastic loading/unloading) }
$$

which are to be finally complemented with the consistency requirement

$$
f\left(\sigma_{i n t}^{\mid e}, k\right)=0 \Rightarrow \dot{\gamma} \dot{f}\left(\sigma_{i n t}^{\mid e}, k\right)=0 .
$$

Using the first addend in Eqs. (2) and (14), the flow rule of Eq. (10) adopts the usual expression in terms of the plastic strain rate [63]

$$
\dot{\varepsilon}_{p}=\dot{\gamma} \operatorname{sign}\left(\sigma_{i n t}^{\mid e}\right) .
$$


The interpretation given in Eq. (10), however, will allow us to extend the formulation to the finite strain context following analogous steps to those followed within the infinitesimal theory [49].

\subsection{Incremental theory}

In the previous section we have derived the set of equations that describe the elastoplastic behavior of the device of Fig. 1. We integrate these equations in the "time" $(t)$ domain by means of the usual elastic predictor/plastic corrector algorithm in order to compute the internal state at $t+\Delta t$, namely ${ }^{t+\Delta t}{ }_{0} \varepsilon_{e}$ and ${ }^{t+\Delta t}{ }_{0} \varepsilon_{p}$, when both the elastoplastic state at $t$, namely ${ }_{0}^{t} \varepsilon={ }_{0}^{t} \varepsilon_{e}+{ }_{0}^{t} \varepsilon_{p}$, and the total strain at $t+\Delta t$, namely ${ }^{t+\Delta t}{ }_{0} \varepsilon$, are known. We use the notation of Ref. [1] for the incremental formulation.

First of all, assume that the incremental deformation between times $t$ and $t+\Delta t$ has been purely elastic. Accordingly, we define the trial elastic strain ${ }^{t r} \varepsilon_{e}$ at instant $t+\Delta t$ as that obtained with the plastic strain retained fixed, i.e. with ${ }^{t r} \varepsilon_{p}={ }_{0}^{t} \varepsilon_{p}$

$$
{ }^{t r} \varepsilon_{e}={ }^{t+\Delta t}{ }_{0} \varepsilon-{ }_{0}^{t} \varepsilon_{p} .
$$

Since ${ }_{0}^{t} \varepsilon_{p}$ remains fixed during the current step, Eq. (18) establishes a one-to-one relation between ${ }^{t+\Delta t}{ }_{0} \varepsilon$ and ${ }^{t r} \varepsilon_{e}$. Hence, for further algorithmic convenience, we can perform a change of the independent variable $\varepsilon$ in Eq. (1) - note the abuse of functional notation for ${ }^{t+\Delta t} \varepsilon_{e}(\dagger, \star)$

$$
{ }_{0}^{t+\Delta t} \varepsilon_{e}\left({ }_{0}^{t+\Delta t} \varepsilon,{ }_{0}^{t+\Delta t} \varepsilon_{p}\right) \rightarrow{ }^{t+\Delta t}{ }_{0} \varepsilon_{e}\left({ }^{t r} \varepsilon_{e},{ }^{t+\Delta t}{ }_{0} \varepsilon_{p}\right)
$$

such that we obtain the following partial derivative of ${ }^{t+\Delta t}{ }_{0} \varepsilon_{e}$ with respect to the new independent variable ${ }^{t r} \varepsilon_{e}-\mathrm{cf}$. the corresponding partial derivative present in Eq. (2)

$$
\left.\frac{\partial^{t+\Delta t}{ }_{0} \varepsilon_{e}}{\partial^{t+\Delta t}{ }_{0} \varepsilon}\right|_{\dot{\varepsilon}_{p}=0}=\left.\frac{\partial^{t+\Delta t}{ }_{0} \varepsilon_{e}}{\partial^{t r} \varepsilon_{e}}\right|_{\dot{\varepsilon}_{p}=0}=1 .
$$

The generalization of the only apparently obvious Eqs. (19) and (20) to the three-dimensional, finite strain context will prove crucial during the integration of the incremental formulation derived below.

In order to determine whether the step has really been elastic, the yield function must be checked with the trial stress, obtained from the trial elastic strain

$$
{ }^{t r} \sigma_{i n t}^{l e}=\left.\frac{d \Psi_{i n t}\left(\varepsilon_{e}\right)}{d \varepsilon_{e}}\right|_{t r}
$$

so

$$
{ }^{t r} f=f\left({ }^{t r} \sigma_{i n t}^{\mid e},{ }^{t r} k\right)=\left({ }^{t r} \sigma_{i n t}^{\mid e}\right)^{2}-{ }^{t r} k^{2}
$$

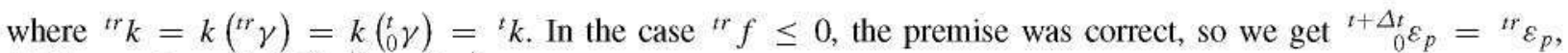
${ }^{t+\Delta t} \varepsilon_{e}={ }^{t r} \varepsilon_{e}$ and ${ }^{t+\Delta t} \sigma_{i n t}^{l e}={ }^{t r} \sigma_{i n t}^{l e}$.

In the case ${ }^{t r} f>0$, a plastic-elastic correction must be performed by means of an internal evolution for which the external strain ${ }^{t+\Delta t}{ }_{0} \varepsilon$ is retained fixed. This is, indeed, the main usefulness of Eq. (10), which we integrate employing a backward-Euler scheme as

$$
{ }^{t+\Delta t}{ }_{0} \varepsilon_{e}-{ }^{t r} \varepsilon_{e}=\left.\int_{t}^{t+\Delta t} d \varepsilon_{e}\right|_{\dot{\varepsilon}=0}=-\int_{t}^{t+\Delta t} \frac{\sigma_{i n t}^{l e}}{k} d \gamma=-\frac{{ }^{t+\Delta t} \sigma_{i n t}^{l e}}{{ }^{t+\Delta t} k} \Delta \gamma
$$

with the nonlinear relations ${ }^{t+\Delta t} k=k\left({ }^{t+\Delta t} \gamma\right)$ and ${ }^{t+\Delta t} \sigma_{i n t}^{l e}=d \Psi_{i n t}\left(\varepsilon_{e}\right) /\left.d \varepsilon_{e}\right|_{t+\Delta t}$. The incremental plastic slip $\Delta \gamma:={ }^{t+\Delta t}{ }_{0} \gamma-{ }^{t r} \gamma={ }^{t+\Delta t} \gamma-{ }_{0}^{t} \gamma$ and the elastic strain ${ }^{t+\Delta t}{ }_{0} \varepsilon_{e}$ are also related from the discrete consistency equation

$$
{ }^{t+\Delta t} f=\left({ }^{t+\Delta t} \sigma_{\text {int }}^{l e}\right)^{2}-{ }^{t+\Delta t} k^{2}=0 .
$$

Both Eqs. (23) and (24) are to be solved in an incremental manner to give the elastoplastic state at $t+\Delta t$. We give below more details about the general iterative procedure employed within the three-dimensional finite strain setting, which remarkably follows the same (simple) steps addressed herein. 


\section{Multiplicative elastoplasticity based on logarithmic strains}

In this section we outline the main ingredients of the novel finite strain continuum framework presented in Ref. [49], which represent the point of departure for the derivation of the computational formulation and that we directly present herein specialized to logarithmic strains. In addition, we extend the finite strain formulation of Ref. [49] to include kinematical hardening effects in an also unusual, yet simpler setting for algorithmic implementation. The main novelty is the derivation of a plastic flow evolution equation expressed in terms of the corrector contribution of an elastic strain rate instead of the usual plastic strain rates. This flow rule, which is valid for anisotropic multiplicative elastoplasticity, arbitrarily large elastic strains and arbitrary yield functions, will result in a remarkably simple additive update for internal logarithmic elastic strains and may be considered the proper generalization to anisotropy in the full strain space of the well-known flow rule for isotropic elastoplasticity derived by Simó and Miehe [51] and Simo [11], which was ultimately written in terms of principal logarithmic strains within the computational part.

\subsection{Multiplicative decomposition}

The so-called Lee multiplicative decomposition [35] states the decomposition of the deformation gradient into an elastic part and a plastic part - we use the symbol - for the usual single index contraction operation

$$
\boldsymbol{X}=\boldsymbol{X}_{e} \cdot \boldsymbol{X}_{p} \cdot
$$

When using this decomposition, a rigid body motion results into

$$
\boldsymbol{X}^{+}=\boldsymbol{Q} \cdot \boldsymbol{X}=\boldsymbol{X}_{e}^{+} \cdot \boldsymbol{X}_{p}^{+}=\left(\boldsymbol{Q} \cdot \boldsymbol{X}_{e}\right) \cdot\left(\boldsymbol{X}_{p}\right)
$$

so the superimposed rigid body motion naturally enters the "elastic" gradient, whereas the plastic gradient remains unaltered. A much debated issue is the uniqueness of the intermediate configuration arising from $X_{p}$ since any rotation tensor $Q$ with its inverse may be inserted such that $\boldsymbol{X}=\left(\boldsymbol{X}_{e} \cdot Q\right) \cdot\left(Q^{T} \cdot \boldsymbol{X}_{p}\right)$. However since $\boldsymbol{X}_{p}$ is path dependent and is integrated step-by-step during the incremental formulation (whether directly as usual, or indirectly as below), we consider that it is uniquely determined at all times [64].

\subsection{Logarithmic strain rate tensors}

Just as an analytical example, consider the total Green-Lagrange strains in the reference configuration and the elastic Green-Lagrange strains in the intermediate configuration obtained from the Lee decomposition of Eq. (25) $I$ stands for the second-order identity tensor

$$
\boldsymbol{A}:=\frac{1}{2}\left(\boldsymbol{X}^{T} \cdot \boldsymbol{X}-\boldsymbol{I}\right) \quad \text { and } \quad \boldsymbol{A}_{e}:=\frac{1}{2}\left(\boldsymbol{X}_{e}^{T} \cdot \boldsymbol{X}_{e}-\boldsymbol{I}\right) .
$$

Following the idea introduced for small strains, see Eq. (1), we write the dependent, internal elastic variable $\boldsymbol{A}_{\ell}$ as a function of the independent, external variable $\boldsymbol{A}$ and the independent, internal plastic variable $\boldsymbol{X}_{p}-$ we use the symbol : for the usual double index contraction operation

$$
\boldsymbol{A}_{e}\left(\boldsymbol{A}, \boldsymbol{X}_{p}\right)=\boldsymbol{X}_{p}^{-T} \cdot\left(\boldsymbol{A}-\boldsymbol{A}_{p}\right) \cdot \boldsymbol{X}_{p}^{-1}=\boldsymbol{X}_{p}^{-T} \odot \boldsymbol{X}_{p}^{-T}:\left(\boldsymbol{A}-\boldsymbol{A}_{p}\right)
$$

where the Green-Lagrange plastic strain tensor is defined in the reference configuration as

$$
\boldsymbol{A}_{p}:=\frac{1}{2}\left(\boldsymbol{X}_{p}^{T} \cdot \boldsymbol{X}_{p}-\boldsymbol{I}\right)
$$

and the symbol $\odot$ performs the mixed dyad product between second-order tensors $(\boldsymbol{Y} \odot \boldsymbol{Z})_{i j k l}=Y_{i k} Z_{j l}$.

Since the one-to-one relations $E_{e}=E_{e}\left(\boldsymbol{A}_{e}\right)$ and $E=E(A)$ hold, where $E_{e}=\frac{1}{2} \ln \left(X_{e}^{T} \cdot X_{e}\right)$ and $E=\frac{1}{2} \ln \left(X^{T} \cdot X\right)$ are the elastic and total material logarithmic strain tensors respectively, we have also the general dependence $\boldsymbol{E}_{e}\left(\boldsymbol{E}, \boldsymbol{X}_{p}\right)$, which generalizes Eq. (1) to the logarithmic strain space. It is important to note that we do not need to know the explicit analytical expression for $\boldsymbol{E}_{e}\left(\boldsymbol{E}, \boldsymbol{X}_{p}\right)$ in the derivation of the present framework. In fact, what we mean with $\boldsymbol{E}_{e}\left(\boldsymbol{E}, \boldsymbol{X}_{p}\right)$ is the also important observation that if we know both $\boldsymbol{E}$ and $\boldsymbol{X}_{p}$, then we can know $\boldsymbol{E}_{e}$, which moreover is uniquely determined. Analogously to Eq. (2), we can decompose the material tensor rate $\dot{E}_{e}$ by means of the addition 
of two partial contributions

$$
\dot{\boldsymbol{E}}_{e}=\left.\frac{\partial \boldsymbol{E}_{e}}{\partial \boldsymbol{E}}\right|_{\dot{X}_{p}=\mathbf{0}}: \dot{\boldsymbol{E}}+\left.\frac{\partial \boldsymbol{E}_{e}}{\partial \boldsymbol{X}_{p}}\right|_{\dot{E}=\mathbf{0}}: \dot{\boldsymbol{X}}_{p}=\left.\dot{\boldsymbol{E}}_{e}\right|_{\dot{\boldsymbol{x}}_{p}=\mathbf{0}}+\left.\dot{\boldsymbol{E}}_{e}\right|_{\dot{E}=\mathbf{0}}
$$

$\mathrm{Eq}$. (30) naturally sets "perpendicular", independent rates within a continuum framework (where they occur both instantaneously and simultaneously) in the sense that the path constrained by $\dot{\boldsymbol{X}}_{p}=\mathbf{0}$ would bring the corresponding stress power without any internal dissipation and the path constrained by $\dot{E}=\mathbf{0}$ would perform the plastic dissipation without exchange of external power. This decomposition in rate form is the origin of the trial elastic predictor, for which $\boldsymbol{X}_{p}$ is frozen, and plastic corrector, for which $\boldsymbol{E}$ is frozen, operator split typically employed for elastic internal variables in computational inelasticity within an algorithmic framework (where they occur incrementally and sequentially). These last considerations will prove very useful below. We want to remark that the general expression of Eq. (30) is fully consistent with the continuum kinematic formulation derived from the Lee multiplicative decomposition and bypasses the definition of any plastic metric, see discussion in Ref. [49].

\subsection{Dissipation in terms of the elastic logarithmic strain rate}

The dissipation inequality may be written in different configurations employing the previous kinematic relations. The stress power is written as [52]

$$
\mathcal{P}=\boldsymbol{S}: \dot{\boldsymbol{A}}=\boldsymbol{T}: \dot{\boldsymbol{E}}=\boldsymbol{\tau}: \boldsymbol{d}
$$

where $S$ is the material second Piola-Kirchhoff stress tensor, work-conjugate of the Green-Lagrange strains $A ; T$ is the so-called material generalized Kirchhoff stress tensor, work-conjugate of the logarithmic strains $E$ in the most general case; and $\boldsymbol{\tau}$ is the spatial Kirchhoff stress tensor, work-conjugate of the Almansi strains $\boldsymbol{a}:=\frac{1}{2}\left(\boldsymbol{I}-\boldsymbol{X}^{-T} \cdot \boldsymbol{X}^{-1}\right.$ ) when its referential configuration, i.e. the current configuration, is maintained fixed, see Refs. [52,49].

The stored energy function may be written in terms of any Lagrangian strain measure, whose selection is just a matter of convenience regarding the hyperelastic relation to be used. Motivated by the small strain rheological model of Fig. 1, the stored energy function may be written in terms of the total logarithmic strains and of the elastic ones as

$$
\Psi\left(\boldsymbol{E}, \boldsymbol{E}_{\boldsymbol{e}}\right)=\Psi_{k i n}\left(\boldsymbol{E}, \boldsymbol{a}_{1} \otimes \boldsymbol{a}_{\mathbf{1}}, \boldsymbol{a}_{2} \otimes \boldsymbol{a}_{2}\right)+\Psi_{i n t}\left(\boldsymbol{E}_{e}, \boldsymbol{a}_{\mathbf{1}} \otimes \boldsymbol{a}_{1}, \boldsymbol{a}_{2} \otimes \boldsymbol{a}_{2}\right)
$$

where we have additionally assumed that the material is orthotropic, with $a_{1}$ and $a_{2}$ (and $a_{3}=a_{1} \times a_{2}$ ) defining the orthogonal preferred directions in both the reference and the intermediate configurations. As a first step in the derivation of more complex formulations including texture evolution [43], which require an experimentally motivated constitutive equation additional to that for the plastic spin $\boldsymbol{w}_{p}=s k w\left(l_{p}\right)$ (with $l_{p}=\dot{\boldsymbol{X}}_{p} \boldsymbol{X}_{p}^{-1}$ being the plastic velocity gradient in the intermediate configuration), see examples in Ref. [65], we assume in this work that the texture of the material is permanent. As we explain below within the variables update phase of the computational algorithm, we are additionally considering $\boldsymbol{w}_{p}=\mathbf{0}$ as a posteriori simplifying assumption once the symmetric flow has been integrated.

The dissipation rate in Lagrangian description is

$$
\mathcal{D}=\mathcal{P}-\dot{\Psi}=\mathcal{P}-\dot{\Psi}_{k i n}-\dot{\Psi}_{i n t} \geq 0
$$

which can be written as

$$
\mathcal{D}=\boldsymbol{T}: \dot{\boldsymbol{E}}-\boldsymbol{T}_{k i n}: \dot{\boldsymbol{E}}-\boldsymbol{T}_{i n t}^{\mid e}: \dot{\boldsymbol{E}}_{e} \geq 0
$$

where we have introduced the following notation for the total derivative (stress) tensors

$$
\boldsymbol{T}_{k i n}:=\frac{d \Psi_{k i n}(\boldsymbol{E})}{d \boldsymbol{E}} \quad \text { and } \quad \boldsymbol{T}_{i n t}^{\mid e}:=\frac{d \Psi_{i n t}\left(\boldsymbol{E}_{e}\right)}{d \boldsymbol{E}_{e}}
$$

with $\boldsymbol{T}_{k i n}$ lying in the reference configuration, as $\boldsymbol{E}$ does, and $\boldsymbol{T}_{i n t}^{\mid e}$ lying in the intermediate configuration, as $\boldsymbol{E}_{e}$ does.

Consider first the case for which $\dot{\boldsymbol{X}}_{p}=\mathbf{0}$, i.e. we have $\left.\dot{\boldsymbol{E}}_{e} \equiv \boldsymbol{E}_{e}\right|_{\dot{\boldsymbol{X}}_{p}=\mathbf{0}}=\partial \boldsymbol{E}_{e} /\left.\partial \boldsymbol{E}\right|_{\dot{\boldsymbol{X}}_{p}=\mathbf{0}}: \dot{\boldsymbol{E}}$ and

$$
\mathcal{D}=\boldsymbol{T}: \dot{\boldsymbol{E}}-\boldsymbol{T}_{k i n}: \dot{\boldsymbol{E}}-\boldsymbol{T}_{i n t}^{\mid e}:\left.\dot{\boldsymbol{E}}_{e}\right|_{\dot{\boldsymbol{X}}_{p}=\mathbf{0}}=0 \quad \text { if } \quad \dot{\boldsymbol{X}}_{p}=\mathbf{0}
$$


so we arrive at - cf. Eq. (6)

$$
\boldsymbol{T}=\boldsymbol{T}_{k i n t}+\boldsymbol{T}_{i n t}^{l e}:\left.\frac{\partial \boldsymbol{E}_{e}}{\partial \boldsymbol{E}}\right|_{\dot{\boldsymbol{x}}_{p}=\mathbf{0}}=\boldsymbol{T}_{k i n}+\boldsymbol{T}_{i n t}
$$

with the partial derivative (stress) tensor - cf. Eq. (7)

$$
\boldsymbol{T}_{i n t}:=\boldsymbol{T}_{i n t}^{\mid e}:\left.\frac{\partial \boldsymbol{E}_{e}}{\partial \boldsymbol{E}}\right|_{\dot{\boldsymbol{X}}_{p}=\mathbf{0}}=\frac{d \Psi_{i n t}\left(\boldsymbol{E}_{e}\right)}{d \boldsymbol{E}_{e}}:\left.\frac{\partial \boldsymbol{E}_{e}}{\partial \boldsymbol{E}}\right|_{\dot{\boldsymbol{X}}_{p}=\mathbf{0}}=\left.\frac{\partial \Psi_{i n t}\left(\boldsymbol{E}_{e}\right)}{\partial \boldsymbol{E}}\right|_{\dot{\boldsymbol{X}}_{p}=\mathbf{0}}
$$

representing the pull-back operation over $T_{i n t}^{l e}$ from the intermediate configuration to the reference configuration. Note that $E_{e}$ is the strain at the intermediate configuration and $\boldsymbol{E}$ is the strain at the material one. Note also that in this case $T_{i n t} \neq T_{i s t}^{\mid e}$ in general, cf. Eq. (6).

On the other side, the dissipation inequality whenever $\dot{\boldsymbol{X}}_{p} \neq \mathbf{0}$ reduces to the general expression $-\mathrm{cf}$. Eq. (8)

$$
\mathcal{D}=-\boldsymbol{T}_{i n t}^{\mid e}:\left.\dot{\boldsymbol{E}}_{e}\right|_{\dot{E}=\mathbf{0}}>0 \text { if } \quad \dot{\boldsymbol{X}}_{p} \neq \mathbf{0}
$$

which, note, is fully expressed in terms of symmetric tensors of purely elastic nature lying in the intermediate configuration, namely the corrector contribution to the elastic logarithmic strain rate $\dot{\boldsymbol{E}}_{e}$ and its power-conjugate generalized Kirchhoff stress tensor $\boldsymbol{T}_{i n t}^{\mid e}$. Remarkably, even though the general dissipation inequality of Eq. (39) is entirely written in the intermediate configuration, note that the traditional non-symmetric Mandel stress tensor, powerconjugate of the non-symmetric plastic velocity gradient tensor, is no longer present in it. As it occurs in the small strain regime and it should do in the continutum-based finite strain one [55], plastic spin effects do not take explicit part in the dissipation inequality and become fully uncoupled from the integration of the evolution equation for the elastic internal strains [49].

\subsection{A six-dinensional evolution equation for natural elastic strains}

The dissipation inequality obtained in Eq. (39) must be positive for all the possible motions according to the second law of thermodynamics, which imposes restrictions on the possible forms of the evolution equations. Our choice herein is the associative flow rule - we include the deviatoric uniaxial factor $2 / 3$ for further convenience

$$
-\left.\dot{\boldsymbol{E}}_{e}\right|_{\dot{E}=0}=\dot{\gamma} \frac{1}{\frac{2}{3} k} \nabla \phi_{T}
$$

where $\dot{\gamma}$ stands for the plastic consistency parameter, $k$ is the internal flow stress parameter and $\phi_{T}\left(T_{i s i}^{\mid e}\right)$ is the Lagrangian internal convex potential

$$
\phi_{T}\left(\boldsymbol{T}_{i n t}^{\mid e}\right)=\frac{1}{2} \boldsymbol{T}_{i n t}^{\mid e}: \mathbb{N}_{T}: \boldsymbol{T}_{i n t}^{\mid e} .
$$

Thus - cf. Eq. (10)

$$
-\left.\dot{E}_{e}\right|_{\dot{E}=0}=\dot{\gamma} \frac{1}{\frac{2}{3} k}\left(\mathbb{N}_{T}: T_{i n t}^{l e}\right)
$$

automatically fulfills the physical requirement

$$
\mathcal{D}=\dot{\gamma} \frac{1}{\frac{2}{3} k} \boldsymbol{T}_{i n t}^{\mid e}: \mathbb{N}_{T}: \boldsymbol{T}_{i n t}^{\mid e}>0 \text { if } \dot{\gamma}>0
$$

when $\mathbb{N}_{T}$ is a positive-definite fully symmetric fourth order tensor.

As we show below, Eq. (40) provides the optimal computational framework for multiplicative elastoplasticity and arguably solves the so-called "rate issue" [11], exactly preserving the classical return mapping schemes of the infinitesimal theory even for the most general orthotropic elastoplasticity case. Indeed, we will be able to directly integrate the corrector contribution to the elastic strain rate tensor present in $\mathrm{Eq}$. (40) in an additive manner in the same way as we did in the infinitesimal setting, recall Eq. (23), which will be physically sound thanks to the intrinsic use of logarithmic strains [56]. 
(i) Multiplicative decomposition of the deformation gradient $\boldsymbol{X}=\boldsymbol{X}_{e} \cdot \boldsymbol{X}_{p}$

(ii) Symmetric elastic strain variable $E_{\varepsilon^{\prime}}=\frac{1}{2} \ln \left(X_{\ell}^{T} \cdot X_{\ell}\right)$

(iii) Kinematics induced by $\boldsymbol{E}_{\ell}\left(\boldsymbol{E}, \boldsymbol{X}_{p}\right)$

$$
\dot{\boldsymbol{E}}_{e}=\left.\dot{E}_{e}\right|_{\dot{X}_{y}=0}+\left.\dot{E}_{e}\right|_{\dot{E}=0} \neq \dot{E}-\dot{E}_{p}
$$

(iv) Symmetric stresses deriving from the stored energy $\Psi\left(\boldsymbol{E}, \boldsymbol{E}_{e}\right)$

$$
\boldsymbol{T}=\frac{\partial \Psi\left(\boldsymbol{E}, \boldsymbol{E}_{e}\right)}{\partial \boldsymbol{E}}=\frac{d \Psi_{k i n}(\boldsymbol{E})}{d \boldsymbol{E}}+\underbrace{\frac{d \Psi_{i n t}\left(\boldsymbol{E}_{e}\right)}{d \boldsymbol{E}_{\ell}}}_{T_{i n t}^{e}}: \frac{\partial \boldsymbol{E}_{e}}{\partial \boldsymbol{E}}=\boldsymbol{T}_{k i n}+\boldsymbol{T}_{i n t}
$$

(v) Evolution equation for associative symmetric plastic flow

$$
\begin{gathered}
\quad-\left.\dot{\boldsymbol{E}}_{k}\right|_{\dot{\boldsymbol{E}}=\mathbf{0}}=\dot{\gamma} \frac{1}{\frac{2}{3} k} \nabla \phi_{T}\left(\boldsymbol{T}_{i m t}^{\mid \ell}\right) \neq \dot{\boldsymbol{E}}_{p} \\
\dot{\gamma} \geq 0, f_{T}\left(\boldsymbol{T}_{i m t}^{\mid \ell}, k\right)=2 \phi_{T}\left(\boldsymbol{T}_{i m t}^{\mid \ell}\right)-\frac{2}{3} k^{2} \leq 0, \dot{\gamma} f_{T}\left(\boldsymbol{T}^{\mid e}, k\right)=0
\end{gathered}
$$

(vi) Additional evolution equation for skew-symmetric plastic fow $w_{p}$

Note: Potentials $\Psi_{\text {kit }}, \Psi_{i m}$ and function $f_{T}$ are anisotropic, in general.

Box I. Finite strain anisotropic hardening multiplicative elastoplasticity model formulated im terms of logarithmic strains.

\subsection{The stem yield function}

The dissipation inequality and the yield function can be expressed in terms of tensor variables lying in any configuration and in terms of any arbitrary pair of stress and strain work-conjugate measures [49]. Our preferences herein (as well as in Refs. $[43,45]$ ) are the elastic logarithmic strains in the intermediate configuration $\boldsymbol{E}_{e}$ and their work-conjugate internal generalized Kirchhoff stresses $T_{i n t}^{l e}$. Consistently, we choose $\mathbb{N}_{T}$ as the specific (stem) tensor of yield constants associated with the preferred material axes in the intermediate configuration, which we denote as the stem configuration as well.

We can rephrase Eq. (43) as

$$
\mathcal{D}=\dot{\gamma} \frac{1}{\frac{2}{3} k}\left(\boldsymbol{T}_{i n t}^{\mid e}: \mathbb{N}_{T}: \boldsymbol{T}_{i n t}^{\mid e}-\frac{2}{3} k^{2}\right)+k \dot{\gamma}>0 \quad \text { if } \quad \dot{\gamma}>0
$$

where we immediately (and naturally) recognize the yield function $f_{T}\left(\boldsymbol{T}_{i n t}^{k}, k\right)$, written in the intermediate configuration in terms of the generalized Kirchhoff stresses $\boldsymbol{T}_{i n t}^{\mid e}$, and the plastic loading condition as

$$
f_{T}\left(\boldsymbol{T}_{i n t}^{\mid e}, k\right):=\boldsymbol{T}_{i n t}^{\mid e}: \mathbb{N}_{T}: \boldsymbol{T}_{i n t}^{\mid e}-\frac{2}{3} k^{2}=0 \quad \text { if } \quad \dot{\gamma}>0
$$

such that the dissipation inequality is finally given in terms of the internal flow stress $k>0$ and its power-conjugate slip rate $\dot{\gamma}>0$ through

$$
\mathcal{D}=k \dot{\gamma}>0 \text { if } \dot{\gamma}>0 \text {. }
$$

The tensor $\mathbb{N}_{T}$ in Eq. (45) may be deviatoric as in Hill's yield function (hence the convenience of the factor $2 / 3$ ) or include volumetric terms for pressure-sensitive plasticity. We refer to Ref. [49] for other possibilities.

Finally, the elastic loading/unloading condition just reads

$$
\dot{\gamma}=0 \quad \text { if } \quad f_{T}\left(\boldsymbol{T}_{i n t}^{\mid e}, k\right)=\boldsymbol{T}_{i n t}^{\mid e}: \mathbb{N}_{T}: \boldsymbol{T}_{i n t}^{\mid e}-\frac{2}{3} k^{2}<0
$$

so we can write

$$
\mathcal{D}=k \dot{\gamma} \geq 0 \quad \text { if } \quad \dot{\gamma} \geq 0 \text {. }
$$

The analyzed material model is summarized in Box I.

\section{Consistent linearization of the continuum theory}

The total external stresses, as given in Eq. (37), include two contributions. The stresses $\boldsymbol{T}_{k i s}$ depend exclusively on the external strain tensor $\boldsymbol{E}$ and are directly calculated from the strain energy function $\Psi_{k i s}(\boldsymbol{E})$ once the logarithmic 
strains $E=\frac{1}{2} \ln \left(X^{T} X\right)$ are obtained from the deformation gradient tensor $X$, cf. Eq. (35) $)_{1}$. The strain energy function $\Psi_{k i n}(E)$ may be determined from experimental data as shown in Section 6 and is associated with the kinematic-type hardening response of the material, as we show in the examples below - see Ref. [59] for a different interpretation based on the Armstrong-Frederick kinematic hardening and the work of Lion [37]. Hence, the linearization of $\boldsymbol{T}_{k \text { in }}(\boldsymbol{E})$ in the reference configuration is trivial and is given by the hyperelastic-type moduli

$$
\mathbb{A}_{k i n}:=\frac{d \boldsymbol{T}_{k i n}}{d \boldsymbol{E}}=\frac{d^{2} \Psi_{k i n}(\boldsymbol{E})}{d \boldsymbol{E} d \boldsymbol{E}}
$$

Thereafter in this section we address the consistent elastoplastic linearization of the tnlly dissipative contribution to the total stresses, i.e. $\boldsymbol{T}_{i n t}$, within the continuum framework. As we show, the procedure is completely parallel to the consistent elastoplastic linearization of the small strain continuum theory.

\subsection{Relation between trial and corrector elastic logarithmic strain rate tensors daring plastic flow}

We assume that the stem yield function is given by Eq. (45). We first note that the internal stress tensor $\boldsymbol{T}_{i n t}^{\text {le }}$ depends on the same independent variables as $\boldsymbol{E}_{e}$, namely $\boldsymbol{E}$ and $\boldsymbol{X}_{p}$, and that $k=k(\gamma)$. Therefore the rate of the yield function given in $\mathrm{Eq}$. (45) may be obtained by applying the chain rule of differentiation as

$$
\dot{f}_{T}=\left.\dot{f}_{T}\right|_{\dot{\boldsymbol{X}}_{p}=\mathbf{0}}+\left.\dot{f}_{T}\right|_{\dot{E}=\mathbf{0}} .
$$

For the first addend, which stands for a trial elastic evolution within the continuum theory, we have - recall Eq. (41)

$$
\left.\frac{1}{2} \dot{f}_{T}\right|_{\dot{X}_{p}=\mathbf{0}}=\nabla \phi_{T}:\left.\dot{\boldsymbol{T}}_{i n t}^{\mid e}\right|_{\dot{\boldsymbol{X}}_{p}=\mathbf{0}}=\nabla \phi_{T}: \mathbb{A}_{i s t}^{\mid e}:\left.\dot{\boldsymbol{E}}_{e}\right|_{\dot{\boldsymbol{X}}_{p}=\mathbf{0}}
$$

where we define the hyperelastic logarithmic constitutive tensor in the intermediate configuration as

$$
\mathrm{A}_{i n t}^{\mid e}:=\frac{d \boldsymbol{T}_{i n t}^{\mid e}}{d \boldsymbol{E}_{e}}=\frac{d^{2} \Psi_{i n t}\left(\boldsymbol{E}_{e}\right)}{d \boldsymbol{E}_{e} d \boldsymbol{E}_{e}}
$$

For the second addend, which stands for a corrector elastic evolution within the continuum theory, in Eq. (50) we have

$$
\left.\frac{1}{2} \dot{f}_{T}\right|_{\dot{E}=\mathbf{0}}=\nabla \phi_{T}:\left.\dot{\boldsymbol{T}}_{i n t}^{\mid e}\right|_{\dot{\boldsymbol{E}}=\mathbf{0}}-\frac{2}{3} k k^{\prime} \dot{\gamma}=\nabla \phi_{T}: \mathbb{A}_{i n t}^{l e}:\left.\dot{\boldsymbol{E}}_{e}\right|_{\dot{E}=\mathbf{0}}-\frac{2}{3} k k^{\prime} \dot{\gamma}
$$

where $k^{i}=d k / d \gamma$ is the derivative of the function $k(\gamma)$ that we consider herein to allow for isotropic hardening. Using Eq. (40) we arrive at the following expression for $\left.\dot{f}_{T}\right|_{\dot{E}=0}$

$$
\left.\frac{1}{2} \dot{f}_{T}\right|_{\dot{E}=0}=-\dot{\gamma} \frac{1}{\frac{2}{3} k}\left(\nabla \phi_{T}: \mathbb{A}_{i s t}^{l e}: \nabla \phi_{T}+\frac{4}{9} k^{2} k^{i}\right) \text {. }
$$

The consistency requirement $\dot{f}_{T}=0$ over Eq. (50) when $\dot{\gamma}>0$ yields the consistency requirement at any time $t$ as

$$
\dot{\gamma} \frac{1}{\frac{2}{3} k}=\left(\frac{\mathbb{A}_{i n t}^{l e}: \nabla \phi_{T}}{\nabla \phi_{T}: \mathbb{A}_{i n t}^{\mid e}: \nabla \phi_{T}+\frac{4}{9} k^{2} k^{\prime}}\right):\left.\dot{\boldsymbol{E}}_{e}\right|_{\dot{\boldsymbol{X}}_{p}=0} \quad \text { if } \quad \dot{\gamma}>0 .
$$

Finally, the combination of Eqs. (55) and (40) yields

$$
\left.\dot{\boldsymbol{E}}_{e}\right|_{\dot{\boldsymbol{E}}=\mathbf{0}}=-\left(\frac{\nabla \phi_{T} \otimes\left(\mathbb{A}_{i n t}^{l e}: \nabla \phi_{T}\right)}{\nabla \phi_{T}: \mathbb{A}_{i n t}^{l e}: \nabla \phi_{T}+\frac{4}{9} k^{2} k^{\prime}}\right):\left.\dot{\boldsymbol{E}}_{e}\right|_{\dot{X}_{p}=\mathbf{0}} \quad \text { if } \quad \dot{\gamma}>0
$$

which establishes the consistent coupling between the rates of the two (a priori) independent variables on which $\boldsymbol{T}_{i n t}^{\mid e}\left(\boldsymbol{E}_{e}\right)$ depends, namely the logarithmic strain tensor $\boldsymbol{E}$ (through $\left.\dot{\boldsymbol{E}}_{e}\right|_{\dot{X}_{p}=0}$, see Eq. (30)) and the plastic deformation gradient $\boldsymbol{X}_{p}$ (through $\left.\dot{E}_{e}\right|_{\dot{E}=0}$, see Eq. (30)), such that the stresses $\boldsymbol{T}_{i n t}^{\mid e}$ remain over the yield surface $f_{T}\left(\boldsymbol{T}_{i n t}^{\mid e}, k\right)=0$ during plastic flow. 


\subsection{Relation between total and trial elastic logarithnic strain rate tensors during plastic flow}

We rephrase now Eq. (30) using Eq. (56) to arrive at

$$
\dot{\boldsymbol{E}}_{e}=\left(\mathbb{I}^{S}-\frac{\nabla \phi_{T} \otimes\left(\mathbb{A}_{i n t}^{l e}: \nabla \phi_{T}\right)}{\nabla \phi_{T}: \mathbb{A}_{i n t}^{l e}: \nabla \phi_{T}+\frac{4}{9} k^{2} k^{r}}\right):\left.\dot{\boldsymbol{E}}_{e}\right|_{\dot{\boldsymbol{X}}_{p}=\mathbf{0}} \quad \text { if } \quad \dot{\gamma}>0
$$

which will facilitate the consistent linearization of the finite strain contintum theory in terms of logarithmic strains in the intermediate configuration.

\subsection{Internal continuum elastoplastic tangent}

The internal (Prandtl-branch-type) contribution to the continuum elastoplastic tangent in the logarithmic strain space relates variations of the generalized Kirchhoff stresses $\boldsymbol{T}_{i n t}$ associated with variations of the external logarithmic strains $\boldsymbol{E}$, both tensors operating in the reference configuration. However, on the one hand, the primary stress tensor from which $\boldsymbol{T}_{i n t}$ is calculated is $\boldsymbol{T}_{i n t}^{\mid e}=d \Psi_{i n t}\left(\boldsymbol{E}_{e}\right) / d \boldsymbol{E}_{e}$, which lies in the actual intermediate configuration (cf. Eq. (37)). On the other side, we have seen that we can relate $\dot{\boldsymbol{E}}$ with $\left.\dot{\boldsymbol{E}}_{e}\right|_{\dot{\boldsymbol{X}}_{y}=\mathbf{0}}$ through the push-forward operation given in the first addend of Eq. (30). Hence, the main core of this constitutive tangent is easily calculated in the intermediate configuration through the stress rate

$$
\dot{\boldsymbol{T}}_{i n t}^{e}=\mathbb{A}_{i n t}^{l e}: \dot{\boldsymbol{E}}_{e}=\mathbb{A}_{e p}^{l e}:\left.\dot{\boldsymbol{E}}_{e}\right|_{\dot{\boldsymbol{X}}_{p}=\mathbf{0}}
$$

i.e.

$$
\mathbb{A}_{e p}^{l e}:=\mathbb{A}_{i n t}^{l e}-\frac{\left(\mathbb{A}_{i n t}^{l e}: \nabla \phi_{T}\right) \otimes\left(\mathbb{A}_{i n t}^{l e}: \nabla \phi_{T}\right)}{\nabla \phi_{T}: \mathbb{A}_{i n t}^{l e}: \nabla \phi_{T}+\frac{4}{9} k^{2} k^{i}}
$$

where we have used Eqs. (52) and (57) and we interpret $\mathbb{A}_{e p}^{l e}$ as the internal continuum elastoplastic tangent, lying in the actual intermediate configuration, which is remarkably identical in structure to the small strains one [63].

However, an important difference arises in this case when it is compared with the infinitesimal framework, where there is no distinction between different referential configurations. In this case, however, the stresses $\boldsymbol{T}_{i n t}$ are defined in the reference configuration whereas the internal stresses $T_{i s t}^{l e}$ lie in the intermediate configuration. An internaldeformation-dependent mapping tensor relates both stress tensors throngh the second addend in Eq. (37), so

$$
\dot{\boldsymbol{T}}_{i n t}=\dot{\boldsymbol{T}}_{i n t}^{\mid e}:\left.\frac{\partial \boldsymbol{E}_{e}}{\partial \boldsymbol{E}}\right|_{\dot{\boldsymbol{x}}_{p}=\mathbf{0}}+\boldsymbol{T}_{i n t}^{\mid e}: \frac{d}{d t}\left(\left.\frac{\partial \boldsymbol{E}_{e}}{\partial \boldsymbol{E}}\right|_{\dot{\boldsymbol{x}}_{p}=\mathbf{0}}\right)
$$

and a one-to-one mapping between the total rates $\dot{\boldsymbol{T}}_{i n t}$ and $\dot{\boldsymbol{T}}_{i n t}^{\text {le }}$ cannot be derived in the continuum framework due to the continuous change of configuration between $\boldsymbol{T}_{i n t}$ and $\boldsymbol{T}_{i n t}^{l e}$. However, consistently with the mapping of the first addend of Eq. (30) between $\dot{E}$ and $\dot{\boldsymbol{E}}_{e}$ (i.e. between one of the independent variables and the dependent one), which is performed with the intermediate configuration assumed fixed, the mapping between their (respective) workconjugate stress rates $\dot{\boldsymbol{T}}_{i n t}$ and $\dot{\boldsymbol{T}}_{i n t}^{\mid e}$ should be also performed with the same kinematic constraint over the intermediate configuration, in a way that the proper pull-back operation over $\mathbb{A}_{e p}^{l e}$, as defined in Eq. (58), is performed from the intermediate to the reference configuration - note that this is basically the concept of Lie derivative [49]. Hence, introducing firstly $\mathrm{Eq} .(58)_{2}$ into the first addend of the right-hand side of $\mathrm{Eq}$. (60) and then considering the first addend of the right-hand side of Eq. (30) we arrive at

$$
\left.\dot{\boldsymbol{T}}_{i n t}\right|_{\dot{\boldsymbol{X}}_{p}=\mathbf{0}}:=\dot{\boldsymbol{T}}_{i n t}^{\mathrm{e}}:\left.\frac{\partial \boldsymbol{E}_{e}}{\partial \boldsymbol{E}}\right|_{\dot{\boldsymbol{X}}_{p}=\mathbf{0}}=\left.\dot{\boldsymbol{E}}_{e}\right|_{\dot{\boldsymbol{X}}_{p}=\mathbf{0}}: \mathbb{A}_{e p}^{l e}:\left.\frac{\partial \boldsymbol{E}_{e}}{\partial \boldsymbol{E}}\right|_{\dot{\boldsymbol{X}}_{p}=\mathbf{0}}=\mathbb{A}_{e p}: \dot{\boldsymbol{E}}
$$

where we define the continuum elastoplastic tangent moduli in the reference configuration $\mathbb{A}_{e p}$ by means of the following pull-back operation over its internal counterpart $\mathbb{A}_{e p}^{l e}$

$$
\mathbb{A}_{e p}:=\left.\frac{\partial \boldsymbol{E}_{e}}{\partial \boldsymbol{E}}\right|_{\dot{X}_{p}=\mathbf{0}} ^{T}: \mathbb{A}_{e p}^{l e}:\left.\frac{\partial \boldsymbol{E}_{e}}{\partial \boldsymbol{E}}\right|_{\dot{\boldsymbol{X}}_{p}=\mathbf{0}}
$$

with $\mathbb{A}_{e p}$ preserving the symmetric associative nature of $\mathbb{A}_{e p}^{l e}$, see $\mathrm{Eq} .(59)$. 
Importantly, the concept introduced in $\mathrm{Eq}$. (61) naturally arises within the corresponding predictor/corrector computational framework derived in the trial intermediate configuration that we obtain below for finite element computations. As we will see, within the algorithmic framework the trial plastic deformation gradient ${ }^{~} \boldsymbol{X}_{p}$ remains constant between global iterations at each integration point. As a result, the trial intermediate configuration and the mapping tensor between $T_{i n t}$ and $T_{i n t}^{l e}$ remain also frozen between iterations, so the respective second addend on the right-hand side of Eq. (60) effectively vanishes. The stresses $T_{i n t}^{\mid e}$ have to be previously mapped from the updated to the trial intermediate configuration. Moreover, the expression of the purely geometrical mapping tensor $\partial \boldsymbol{E}_{e} /\left.\partial \boldsymbol{E}\right|_{\dot{\boldsymbol{X}}_{p}=0}$, present in $\mathrm{Eq} .(62)$, is neither needed if the algorithmic linearization is fully derived in the trial intermediate configuration. We refer to Ref. [60] for a very similar consistent algorithmic linearization in the context of anisotropic multiplicative viscoelasticity based on logarithmic strains in the intermediate configuration.

\section{Large strain algorithmic formulation}

We draw now our attention to the algorithmic formulation that derives from the previous continuum formulation. The computation of $\boldsymbol{T}_{k i n}$ and their corresponding tangent moduli are carried out by straightforward evaluations of the hyperelastic law $\Psi_{k i n}(E)$ (i.e. first and second derivatives) once the total strain tensor $E$ is known at each step/iteration from the polar decomposition of the total deformation gradient tensor $\boldsymbol{X}$. Hence, they are obtained in the same way as the equilibrated contributions of the model of Ref. [60], see Section 6 therein, so we give no additional details about them.

Thereafter in this section we address the procedure to obtain the truly dissipative contribution to the total stresses, i.e. $T_{i n t}$, and their consistent elastoplastic tangent moduli within the algorithmic framework. The procedure becomes remarkably simple and preserves the appealing additive structure of the classical return mapping schemes of the small strain theory, being at the same time fully consistent with the Lee factorization of the deformation gradient. A fully symmetric algorithmic linearization is obtained.

\subsection{Stress-point integration algorithm}

We develop herein the stress-point integration algorithm and the consistent algorithmic tangent moduli for finite element analysis in the space of logarithmic strains. This framework is specially useful because of the relevant features of these strain measures in the integration procedure. The large strain kinematics is essentially staggered in a previous pre-processor and an ulterior geometric post-processor.

\subsubsection{Geometric pre-processor: elastic predictor}

Assume that the total deformation gradient ${ }^{t+\Delta t} \boldsymbol{X}$ is given at the step $t+\Delta t$ and that the plastic deformation gradient ${ }_{n}^{t} \boldsymbol{X}_{p}$ is also known by integration of the previous steps (obviously we also know ${ }_{0}^{t} \boldsymbol{X}_{e}$ ). First of all, we define the trial elastic deformation gradient at $t+\Delta t$ as that obtained from the Lee multiplicative decomposition with the plastic deformation gradient frozen, i.e. that for which ${ }^{t r} \boldsymbol{X}_{p}={ }_{0}^{t} \boldsymbol{X}_{p}$

$$
{ }^{t r} \boldsymbol{X}_{e}={ }^{t+\Delta t} \boldsymbol{X} \cdot{ }_{0}^{t} \boldsymbol{X}_{p}^{-1} .
$$

The elastic logarithmic strains associated with this gradient are

$$
{ }^{r} \boldsymbol{E}_{e}=\frac{1}{2} \ln \left({ }^{t r} \boldsymbol{X}_{e}^{T} \cdot{ }^{r r} \boldsymbol{X}_{e}\right) \text {. }
$$

This algorithmic strain tensor resides in the trial intermediate configuration at time $t+\Delta t$ or, in other words, in the updated (converged) intermediate configuration at time $t$, see Eq. (63). Note that these strains inherently correspond to the exact integration of the trial elastic strain rate tensor, i.e. the first addend on the right-hand sides of Eq. (30).

In order to determine whether the incremental loading is elastic or not, the yield function of $\mathrm{Eq}$. (45) must be assessed with the trial stresses in the fixed intermediate configuration, obtained from the trial elastic strains

$$
{ }^{r} \boldsymbol{T}_{i n t}^{l e}:=\left.\frac{d \Psi_{i n t}\left(\boldsymbol{E}_{e}\right)}{d \boldsymbol{E}_{e}}\right|_{t r}=\frac{d \Psi_{i n t}\left({ }^{r} \boldsymbol{E}_{e}\right)}{d^{r} \boldsymbol{E}_{e}} .
$$

Then the yield function is

$$
{ }^{r r} f_{T}=f_{T}\left({ }^{r r} \boldsymbol{E}_{e},{ }^{r r} k\right)={ }^{r r} \boldsymbol{T}_{i n t}^{\mid e}: \mathbb{N}_{T}:{ }^{r r} \boldsymbol{T}_{i n t}^{\mid e}-\frac{2}{3}{ }^{t r} k^{2}
$$


where ${ }^{t r} k=k\left({ }^{t r} \gamma\right)=k\left({ }_{0}^{t} \gamma\right)={ }^{t} k$. Note that ${ }^{t r} f_{T}$ is an invariant; i.e. it can be computed in any configuration using the proper tensors. In the case ${ }^{t r} f_{T} \leq 0$, the step has been elastic and the trial state represents the solution at $t+\Delta t$. In the case ${ }^{t r} f_{T}>0$, the elastic solution is not admissible and the plastic correction must be computed.

\subsubsection{Local Newton iterations: plastic corrector (of the elastic strains)}

In the case ${ }^{t r} f_{T}>0$, the external deformation is frozen and we perform the subsequent internal correction for $\boldsymbol{E}_{e}$. We remark that this substep is usually performed directly over plastic strain variables. However, note that we are explicitly integrating the elastic strain evolution rather than the plastic strain evolution (obviously, they are internally connected). In other words, we integrate in this substep the corrector elastic strain rate tensor, i.e. the second addend on the right-hand sides of Eq. (30). This is, in fact, the key characteristic of the present return mapping scheme that makes it so appealing from an algorithmic standpoint and that makes the difference with current anisotropic multiplicative elastoplasticity computational models based on explicit internal plastic evolutions.

The numerical implementation is based on the backward-Euler Closest Point Projection algorithm. There are several possible implementations of this algorithm [66]. However, we develop here one based on the residual of both elastic strains and yield function. The solution variables are the elastic strains ${ }^{t+}{ }^{t}{ }_{0} E_{e}$ and the consistency parameter ${ }^{t+\Delta t}{ }_{0} \gamma$. The first residue is readily obtained through the integration of Eq. (40) employing a first-order accurate backward-Euler scheme, which results into the following additive update - note that, as in the infinitesimal setting, see Eq. (23), we bypass the explicit use of any exponential mapping thanks to the intrinsic use of logarithmic (natural) strains by the model [56]

$$
\left.\int_{t}^{t+\Delta t} d \boldsymbol{E}_{e}\right|_{\dot{E}=0}=-\int_{t}^{t+\Delta t} \frac{1}{\frac{2}{3} k} \nabla \phi_{T} d \gamma
$$

i.e.

$$
{ }_{0}^{t+\Delta t} \boldsymbol{E}_{e}-{ }^{r} \boldsymbol{E}_{e}=-\frac{\Delta y}{\frac{\pi}{3}^{t+\Delta t} k}{ }^{t+\Delta t} \nabla \phi_{T}
$$

where ${ }^{t+\Delta t} k=k\left({ }^{t+\Delta t} \gamma\right)$ and

$$
{ }^{t+\Delta t} \nabla \phi_{T}=\mathbb{N}_{T}:{ }^{t+\Delta t} \boldsymbol{T}_{i n t}^{\mid e}
$$

with - note that this stress tensor resides in the (yet unconverged) intermediate configuration at time $t+\Delta t$

$$
{ }^{t+\Delta t} \boldsymbol{T}_{i n t}^{\mid e}=\left.\frac{d \Psi_{i n t}\left(\boldsymbol{E}_{e}\right)}{d \boldsymbol{E}_{e}}\right|_{t+\Delta t}
$$

which yields the residual elastic strain tensor

$$
{ }^{t+\Delta t} \boldsymbol{\rho}_{e}={ }^{t+\Delta t} \boldsymbol{E}_{e}+\frac{\Delta \gamma}{\frac{2^{2} t+\Delta t}{3}}{ }^{t+\Delta t} \nabla \phi_{T}-{ }^{t r} \boldsymbol{E}_{e} \longrightarrow \mathbf{0} .
$$

The incremental plastic slip $\Delta \gamma:={ }^{t+\Delta t} \gamma-{ }_{0}{ }^{t r} \gamma={ }^{t+}+{ }_{0}^{t} \gamma-{ }_{0}^{t} \gamma$ and the elastic strains ${ }^{t+}{ }_{0}^{t} \boldsymbol{E}_{e}$ may also be related from the discrete consistency equation ${ }^{t+\Delta t} f_{T} \longrightarrow 0$, which constitutes the second residual equation

$$
{ }^{t+\Delta t} f_{T}={ }^{t+\Delta t} \boldsymbol{T}_{i n t}^{\mid e}:{ }^{t+\Delta t} \nabla \phi_{T}-\frac{2}{3}{ }^{t+\Delta t} k^{2} \longrightarrow 0 .
$$

We write the following vector form

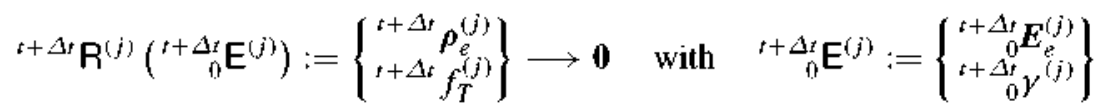

where $(j)$ stands for the local iteration counter. The residual vector is solved in an incremental manner, as usual

$$
{ }^{t+\Delta t} \mathrm{E}^{(j+1)}={ }^{t+\Delta t}{ }_{0} \mathrm{E}^{(j)}-\left[\nabla^{t+\Delta t} \mathrm{R}^{(j)}\right]^{-1}{ }^{t+\Delta t} \mathrm{R}^{(j)}
$$

until

$$
\left\|{ }^{t+\Delta^{t}} \mathrm{R}^{(j+1)}\left({ }^{t+\Delta t} \mathrm{E}^{(j+1)}\right)\right\|<\text { tolerance }
$$


where the starting values $\left(j=0\right.$ ) are the trial ones, i.e. ${ }^{t+}{ }_{n}^{\Delta t} \boldsymbol{E}_{e}^{(0)}={ }^{t r} \boldsymbol{E}_{e}$ and ${ }^{t+\Delta t}{ }_{0} \gamma^{(0)}={ }^{t} \gamma$, and the acceptable solutions must fulfill $\Delta y>0$. The gradient is

$$
\nabla^{t+\Delta t} \mathrm{R}^{(j)}=\left[\begin{array}{ll}
\partial \rho_{e} / \partial \boldsymbol{E}_{e} & \partial \rho_{e} / \partial \gamma \\
\partial f_{T} / \partial \boldsymbol{E}_{e} & \partial f_{T} / \partial \gamma
\end{array}\right]_{t+\Delta t}^{(j)}
$$

which contains the following derivatives evaluated at step $t+\Delta t$ and local iteration $(j)$

- $\partial \rho_{e} / \partial E_{e}$ can be obtained from Eq. (71) as

$$
\frac{\partial \rho_{e}}{\partial \boldsymbol{E}_{e}}=\mathbb{I}^{S}+\frac{\Delta \gamma}{\frac{2}{3} k} \nabla \nabla \phi_{T}: \frac{d \boldsymbol{T}_{i n t}^{\mid e}}{d \boldsymbol{E}_{e}}=\mathbb{I}^{S}+\frac{\Delta \gamma}{\frac{2}{3} k} \mathbb{N}_{T}: \mathbb{A}_{i n t}^{\mid e}
$$

where $\mathbb{A}_{i n t}^{e}$ is the hyperelastic constitutive tangent tensor as given in Eq. (52).

- $\partial \rho_{e} / \partial \gamma$ is readily obtained also from Eq. (71) as

$$
\frac{\partial \rho_{e}}{\partial y}=\frac{k-\Delta \gamma k^{\prime}}{\frac{2}{3} k^{2}} \nabla \phi_{T}=\frac{k-\Delta \gamma k^{\prime}}{\frac{2}{3} k^{2}} \mathbb{N}_{T}: \boldsymbol{T}_{i s t}^{\mid e}
$$

- $\partial f_{T} / \partial E_{e}$ is obtained from Eq. (45) as

$$
\frac{\partial f_{T}}{\partial \boldsymbol{E}_{e}}=2 \nabla \phi_{T}: \frac{d \boldsymbol{T}_{i n t}^{\mid e}}{d \boldsymbol{E}_{e}}=2 \boldsymbol{T}_{i n t}^{\mid e}: \mathbb{N}_{T}: \mathbb{A}_{i n t}^{\mid e}
$$

- $\partial f_{T} / \partial y$ is a scalar derivative which simply contains

$$
\frac{\partial f_{T}}{\partial \gamma}=-\frac{4}{3} k k^{\prime}
$$

Remarkably, the resulting Closest Point Projection algorithm for this large strain anisotropic model with mixed hardening has dimension of seven; i.e. there are no additional internal plastic variables involved $[63,66]$.

\subsubsection{Geometric post-processor: Variables update}

Once the iterative algorithm has converged, we know ${ }^{t+\Delta t}{ }_{0}$ and ${ }^{t+\Delta t} \gamma$. Then we can readily obtain the internal stresses ${ }^{t+\Delta t} \boldsymbol{T}_{i n t}^{k^{e}}$ in the updated intermediate configuration at $t+\Delta t$ through Eq. (70). They must be further pulled back to the reference configuration employing the proper mapping tensors. As already done in Ref. [60], it is algorithmically convenient to perform the pull-back of internal variables from the updated intermediate configuration to the reference configuration via the trial intermediate configuration, which remains fixed during each global step and may be seen as a modified reference configuration. As shown in Ref. [60], recall also Eq. (18), there exists a one-to-one relation between the strain tensor ${ }^{t+}{ }_{0}^{t} E$ (lying in the reference configuration) and the strain tensor ${ }^{t r} E_{e}$ (lying in the trial intermediate configuration), thereby the latter one may be conceptually understood as a modified independent variable on which ${ }^{t+\Delta t} E_{e}$ depends, i.e. we can perform the following change of independent variable, recall Eq. (19)

$$
{ }_{0}^{t+\Delta t} \boldsymbol{E}_{e}\left({ }^{t+\Delta t} \boldsymbol{E},{ }_{0}^{t+\Delta t}{ }_{0} \boldsymbol{X}_{p}\right) \rightarrow{ }^{t+\Delta t} \boldsymbol{E}_{e}\left({ }^{t r} \boldsymbol{E}_{e},{ }^{t+\Delta t} \boldsymbol{X}_{p}\right) .
$$

Thus, in the same way as ${ }^{t+\Delta^{t}} \boldsymbol{T}_{i n t}$ is obtained from the pull-back of ${ }^{t+\Delta t} \boldsymbol{T}_{i n t}^{\mid e}$ to the reference, fixed configuration, see Eq. (38), we can define the stress tensor ${ }^{t+\Delta^{t}} \boldsymbol{T}_{i n t}^{\mid t r}$ as the pull-back of ${ }^{t+\Delta^{t}} \boldsymbol{T}_{i n t}^{e}$ to the trial, fixed configuration (i.e. the intermediate configuration at time $t$ ) through - cf. Eq. (38)

$$
\begin{aligned}
{ }^{t+\Delta t} \boldsymbol{T}_{i n t}^{\mid t r} & =\left.\frac{\partial \Psi_{i n t}\left(\boldsymbol{E}_{e}\right)}{\partial{ }^{t r} \boldsymbol{E}_{e}}\right|_{\dot{\boldsymbol{X}}_{p}=\mathbf{0}} \\
& =\left.\frac{d \Psi_{i n t}\left(\boldsymbol{E}_{e}\right)}{d \boldsymbol{E}_{e}}\right|_{t+\Delta t}:\left.\frac{\partial^{t+\Delta t} \boldsymbol{E}_{e}}{\partial^{t r} \boldsymbol{E}_{e}}\right|_{\dot{\boldsymbol{X}}_{p}=\mathbf{0}} \\
& \simeq{ }^{t+\Delta t} \boldsymbol{T}_{i n t}^{\mid e}: \mathbb{I}^{S}={ }^{t+\Delta t} \boldsymbol{T}_{i n t}^{\mid e}
\end{aligned}
$$

where the fourth-order partial gradient tensor $\partial^{t+\Delta t} \boldsymbol{E}_{e} /\left.\partial^{t r} \boldsymbol{E}_{e}\right|_{\dot{\boldsymbol{X}}_{p}=0}$ is approximately $\mathbb{I}^{S}$ for small plastic increments $\Delta y$ under generic loadings and is exactly $\mathbb{I}^{S}$ for purely axial (rotationless) states thanks to the unique properties of 
the logarithmic strains [56]. Indeed, in the unidimensional case it is exactly the identity, recall Eq. (20). We give more details about this approximation below, see also the discussion in Ref. [60]. We also note that ${ }^{t+\Delta t} \boldsymbol{T}_{i n t}^{\mid t r}$ in Eq. (82), which represents the updated (final) stress tensor ${ }^{t+\Delta t} \boldsymbol{T}_{i n t}^{\mid e}$ mapped to the trial configuration, and ${ }^{t r} \boldsymbol{T}_{i n t}^{e}$ in Eq. (65), evaluated directly in the trial configuration with ${ }^{t r} \boldsymbol{E}_{e}$, are both conceptually and numerically different, which may be easily verified in the unidimensional (linearized) case of Section 2.2. That is, ${ }^{t+\Delta t} \boldsymbol{T}_{i n t}^{\mid t r}$ are the converged stresses modified by a convenient algorithmic, purely geometric mapping and ${ }^{t r} \boldsymbol{T}_{i n t}^{\mid e}$ are the (unconverged) stresses at iteration (0). Then it is straightforward to perform another geometric mapping [52] to obtain the respective second Piola-Kirchhoff stresses in the referential, trial intermediate configuration for quadratic measures - cf. Eq. (103) in Ref. [60]

$$
{ }^{t+\Delta t} \boldsymbol{S}_{i n t}^{\mid t r}={ }^{t+\Delta t} \boldsymbol{T}_{i n t}^{\mid t r}: \frac{d^{t r} \boldsymbol{E}_{e}}{d^{t r} \boldsymbol{A}_{e}}
$$

which are subsequently mapped to the reference configuration through the fourth-order total gradient tensor $d^{t r} \boldsymbol{A}_{e} / d^{t+\Delta t}{ }_{0} \boldsymbol{A}-$ cf. Eq. (100) in Ref. [60]

$$
{ }^{t+\Delta t} \boldsymbol{S}_{i n t}={ }^{t+\Delta t} \boldsymbol{S}_{i n t}^{t r}: \frac{d^{t r} \boldsymbol{A}_{e}}{d^{t+\Delta t}{ }_{0} \boldsymbol{A}}={ }^{t+\Delta t} \boldsymbol{S}_{i n t}^{\mid t r}:{ }^{t r} \boldsymbol{X}_{p}^{-T} \odot{ }^{t r} \boldsymbol{X}_{p}^{-T}={ }_{0}^{t} \boldsymbol{X}_{p}^{-1} \cdot{ }^{t+\Delta t} \boldsymbol{S}_{i n t}^{\mid t r} \cdot{ }_{0}^{t} \boldsymbol{X}_{p}^{-T} .
$$

At this point, we note that the associative six-dimensional flow rule given in Eq. (40) has been integrated independently of the plastic spin evolution [55,49], which is again another salient feature of the present model, inherited at the same time from the infinitesimal model. Thus, after the integration of the symmetric flow the intermediate configuration, defined by ${ }_{0}^{t+\Delta t} \boldsymbol{X}_{p}$, remains undetermined up to an arbitrary finite rotation ${ }^{t+\Delta t} \boldsymbol{R}_{e}[18]$, which we must finally update during the convergence phase at $t+\Delta t$ for the computation of the next incremental load step and to complete the formulation. As we did in Ref. [60], we update the elastic deformation gradient tensor through

$$
{ }^{t+\Delta t} \boldsymbol{X}_{e}={ }^{t+\Delta t}{ }_{0}^{\Delta t} \boldsymbol{R}_{e} \cdot{ }^{t+\Delta t} \boldsymbol{U}_{e}={ }^{t+\Delta t}{ }_{0} \boldsymbol{R}_{e} \cdot \exp \left({ }^{t+\Delta t}{ }_{0} \boldsymbol{E}_{e}\right)
$$

where we take ${ }^{t+\Delta t}{ }_{0} \boldsymbol{R}_{e}={ }^{t r} \boldsymbol{R}_{e}$, i.e. we assume that the elastic rotation tensor is not modified during plastic flow. This assumption is the same to that in [21,45], but in the present context it is merely a posteriori prescription for the skew-symmetric flow, not a consequence of any needed approximation for the integration of the nine-dimensional evolution of the plastic deformation gradient [49]. Hence this assumption may be modified to accommodate any other plastic spin evolution and/or texture evolution if desired [43] in a completely uncoupled fashion, i.e. after the symmetric flow is integrated. The plastic deformation gradient may be recovered if desired

$$
{ }^{t+\Delta t} \boldsymbol{X}_{p}={ }^{t+\Delta t}{ }_{0}^{-1} \cdot{ }^{t+\Delta t} \boldsymbol{X} .
$$

\section{Remark}

The relation between ${ }^{t+\Delta t}{ }_{0} \boldsymbol{R}_{e},{ }^{t r} \boldsymbol{R}_{e}$ and the plastic spin ${ }^{t+\Delta t} \boldsymbol{w}_{p}=s k w\left({ }^{t+\Delta t} \boldsymbol{l}_{p}\right)$, with $\boldsymbol{l}_{p}=\dot{\boldsymbol{X}}_{p} \boldsymbol{X}_{p}^{-1}$ being the plastic velocity gradient in the intermediate configuration, is given by Eq. (28) in Ref. [43]

$$
{ }^{t+\Delta t} \boldsymbol{R}_{e} \simeq{ }^{t r} \boldsymbol{R}_{e}{ }^{t+\Delta t}{ }_{t}^{T} \boldsymbol{R}_{w}^{T}
$$

where ${ }^{t+\Delta t}{ }_{t} \boldsymbol{R}_{w}$ is the incremental plastic rotation - Eq. (17) in Ref. [43]

$$
{ }^{t+\Delta t} \boldsymbol{R}_{w}=\exp \left(\Delta t{ }^{t+\Delta t} \boldsymbol{w}_{p}\right) .
$$

Hence, the assumption ${ }^{t+\Delta t}{ }_{0} \boldsymbol{R}_{e}={ }^{t r} \boldsymbol{R}_{e}$ taken above is equivalent to the common assumption in computational anisotropic elastoplasticity models ${ }^{t+\Delta t} \boldsymbol{w}_{p}=\mathbf{0}$, i.e. a vanishing plastic spin in the intermediate configuration.

If a plastic spin evolution $\boldsymbol{w}_{p}$ is prescribed as an additional constitutive equation, then we know ${ }^{t+\Delta t}{ }_{t}^{t} \boldsymbol{R}_{w}$ through Eq. (87), which should be considered in the update of the elastic deformation gradient tensor. In this case (nonvanishing plastic spin), the definition of a rotationally-frozen intermediate configuration would greatly facilitate the integration of the symmetric internal flow and the consistent linearization of the algorithm [43]. 


\subsection{Plastic volume presentation}

If a pressure insensitive yield criterion is considered, namely $\operatorname{tr}\left(\nabla \phi_{T}\right)=\nabla \phi_{T}: \boldsymbol{I}=0$, then Eq. (68) gives

$$
\ln \left({ }^{t+\Delta_{0} t} J_{e}\right)=\operatorname{tr}\left({ }^{t+\Delta_{0} t} \boldsymbol{E}_{e}\right)=\operatorname{tr}\left({ }^{t r} \boldsymbol{E}_{e}\right)=\ln \left({ }^{t r} J_{e}\right)
$$

i.e. ${ }^{t+\Delta t} J_{e}={ }^{t r} J_{e}$, where $J_{e}:=\operatorname{det}\left(X_{e}\right)$. From Eqs. (63) and (85), we immediately arrive at

$$
{ }_{0}^{t+\Delta t} J_{p}=\operatorname{det}\left({ }^{t+\Delta t} \boldsymbol{X}_{p}\right)=\operatorname{det}\left({ }_{0}^{t} \boldsymbol{X}_{p}\right)={ }_{0}^{t} J_{p}
$$

so the present algorithmic procedure automatically preserves plastic volume in that case.

\subsection{Special cases of isotropy}

In the most general case of fully anisotropic elastoplasticity, the plastic potential gradient ${ }^{t+\Delta t} \nabla \phi_{T}$ of $\mathrm{Eq}$. (69) does not commute with ${ }^{t+\Delta t} T_{i n t}^{l e}$ or ${ }^{t+\Delta t} E_{e}$, so the terms in Eq. (68) are not coaxial and Eq. (68) is effectively six-dimensional, in general. For isotropic elasticity along with anisotropic plasticity responses, ${ }^{t+\Delta t} \boldsymbol{T}_{\text {int }}^{\mid e}$ and ${ }^{t+\Delta t} \boldsymbol{E}_{e}$ commute but the presence of $\mathbb{N}_{T}$ in ${ }^{t+\Delta t} \nabla \phi_{T}$ involves a rotation of the elastic strain/stress principal directions (from trial to updated) in Eq. (68), which still remains six-dimensional. For a fully isotropic elastoplasticity response, all the terms in $\mathrm{Eq} .(68)$ commute, so the return mapping takes place at fixed, trial principal axes and we recover the well-known three-dimensional additive update in principal logarithmic strain space of Ref. [11], but written in this case in terms of elastically rotated variables.

The advantage of the formulation presented in this paper, which hinges on the application of the novel elastic strain corrector-type evolution equation given in $\mathrm{Eq}$. (68), should be apparent now because, having been derived following a very simple and clear procedure, it provides a more general, fully anisotropic, formulation than the works of Simó [11], Eterovic and Bathe [21] and Weber and Anand [20]. In addition, due to its remarkable similarity with the infinitesimal case, the present formulation also becomes simpler than current computational anisotropic elastoplasticity models.

\subsection{Consistent algorithmic moduli}

The main core of the consistent algorithmic elastoplastic tangent tensor may now be calculated in the trial intermediate configuration, which remains fixed during the current step, through — note that this tangent moduli tensor, which is defined in the trial intermediate configuration, is the algorithmic counterpart of the continum elastoplastic tangent $\mathbb{A}_{e p}^{\mid e}$ given in $\mathrm{Eq}$. (58), which is defined in the updated intermediate configuration

$$
\begin{aligned}
{ }^{t+\Delta t} \mathbb{A}_{e p}^{\mid t r}: & =\frac{d^{t+\Delta t} \boldsymbol{T}_{i n t}^{\mid t r}}{d^{t r} \boldsymbol{E}_{e}} \simeq \frac{d^{t+\Delta t} \boldsymbol{T}_{i n t}^{\mid e}}{d^{t r} \boldsymbol{E}_{e}} \\
& =\left.\frac{d \boldsymbol{T}_{i n t}^{l e}\left(\boldsymbol{E}_{e}\right)}{d \boldsymbol{E}_{e}}\right|_{t+\Delta t}: \frac{d^{t+\Delta t} \boldsymbol{E}_{e}}{d^{t r} \boldsymbol{E}_{e}}={ }^{t+\Delta t} \mathbb{A}_{i n t}^{\mid e}: \frac{d^{t+\Delta t} \boldsymbol{E}_{e}}{d^{t r} \boldsymbol{E}_{e}}
\end{aligned}
$$

which is a linearization consistent with the approximation of $\mathrm{Eq} .(82)$. We also remark the difference between the

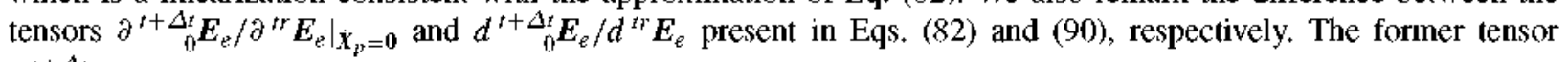
$\partial^{t+\Delta t} \boldsymbol{E}_{e} /\left.\partial^{t r} \boldsymbol{E}_{e}\right|_{\dot{\boldsymbol{X}}_{p}=0}$ operates during a hypothetical situation in which the updated configuration is retained frozen, i.e. in which ${ }^{t+\Delta t} \dot{X}_{p}=\mathbf{0}$; it converges to the symmetric projector tensor $\mathbb{I}^{S}$ within the continuum theory, i.e. when $\Delta y \rightarrow 0$; and it is used to compute stresses, which involve the condition ${ }^{t+\Delta t} \dot{\boldsymbol{X}}_{p}=\mathbf{0}$ by definition, see for example Eq. (38). On the contrary, the tensor $d^{t+\Delta t} \boldsymbol{E}_{e} / d^{t r} \boldsymbol{E}_{e}$ operates when plastic flow is taking place, i.e. when ${ }^{t+\Delta t} \dot{\boldsymbol{X}}_{p} \neq \mathbf{0}$; it converges to the fourth-order tensor present in Eq. (57) in the limit of the continuum theory, as we show below; and it is used to compute derivatives of stresses during plastic flow, i.e. elastoplastic tangent moduli, which must include a plastic correction contribution additional to the elastic predictor contribution.

The fourth-order tensor for the finite increment $d^{t+\Delta t} \boldsymbol{E}_{e} / d^{t r} \boldsymbol{E}_{e}$ must be obtained in this case from the backwardEuler stress integration algorithm employed above, as we show next. The first residue given in Eq. (71) brings the following Equation because ${ }^{t+\Delta t} \rho_{e}=\mathbf{0}$ holds between global iterations at step $t+\Delta t$

$$
\mathbb{O}=\frac{d^{t+\Delta t} \rho_{e}}{d^{t r} \boldsymbol{E}_{e}}
$$


so

$$
\bigoplus=\frac{\partial^{t+\Delta t} \boldsymbol{\rho}_{e}}{\partial^{t+\Delta t} \boldsymbol{E}_{e}}: \frac{d^{t+\Delta t} \boldsymbol{E}_{e}}{d^{t r} \boldsymbol{E}_{e}}+{ }^{t+\Delta t} \nabla \phi_{T} \otimes \frac{d \Gamma}{d^{t r} \boldsymbol{E}_{e}}-\mathbb{I}^{S}
$$

with the following definition $\Gamma(\gamma):=\frac{3}{2} \Delta \gamma / k(\gamma)$. The tenn $\partial^{t+\Delta t} \rho_{e} / \partial^{t+\Delta t} E_{e}$ is

$$
{ }^{t+\Delta t} \mathbb{K}:=\frac{\partial^{t+\Delta t} \rho_{e}}{\partial^{t+\Delta t}{ }_{0} E_{e}}=\left(\mathbb{I}^{s}+{\frac{\Delta \gamma}{\frac{2}{3}^{t+\Delta t} k}}^{t+\Delta t} \nabla \nabla \phi_{T}: \frac{d^{t+\Delta t} \boldsymbol{T}_{i n t}^{\mid e}}{d^{t+\Delta t} \boldsymbol{E}_{e}}\right)
$$

and is known from Eq. (77) once the local iterations have converged at each global iteration. Thus we can factor-out

$$
\frac{d^{t+\Delta t} \boldsymbol{E}_{e}}{d^{t r} \boldsymbol{E}_{e}}={ }^{t+\Delta t} \mathbb{K}^{-1}:\left(\mathbb{I}^{S}-{ }^{t+\Delta t} \nabla \phi_{T} \otimes \frac{d^{t+\Delta t} \Gamma}{d^{t r} \boldsymbol{E}_{e}}\right)
$$

From this expression only $d^{t+\Delta t} \Gamma / d^{t r} \boldsymbol{E}_{e}$ is still unknown. Due to the fact that the yield condition ${ }^{t+\Delta t} f_{T}=0$ (second residual equation) is also fulfilled between global iterations, we have

$$
\mathbf{0}=\frac{1}{2} \frac{d^{t+\Delta t} f_{T}}{d^{t r} \boldsymbol{E}_{e}}={ }^{t+\Delta t} \nabla \phi_{T}: \frac{d^{t+\Delta t} \boldsymbol{T}_{i n t}^{\mid e}}{d^{t+\Delta t} \boldsymbol{E}_{e}}: \frac{d^{t+\Delta t} \boldsymbol{E}_{e}}{d^{t r} \boldsymbol{E}_{e}}-\frac{2}{3} k k^{\prime} \frac{d \Delta \gamma}{d^{t r} \boldsymbol{E}_{e}}
$$

so, using Eq. (94)

$$
\mathbf{0}={ }^{t+\Delta t} \nabla \phi_{T}:{ }^{t+\Delta t} \mathbb{D}-\left({ }^{t+\Delta t} \nabla \phi_{T}:{ }^{t+\Delta t} \mathbb{D}:{ }^{t+\Delta t} \nabla \phi_{T}\right) \frac{d^{t+\Delta t} \Gamma}{d^{t r} \boldsymbol{E}_{e}}-\frac{\mathbf{4}}{3} \frac{k^{2} k^{i}}{3-2 \Gamma k^{\prime}} \frac{d^{t+\Delta^{t}} \Gamma}{d^{t r} \boldsymbol{E}_{e}}
$$

where we define the elastic algorithnic moduli ${ }^{t+\Delta t} \mathbb{D}$ as - note that ${ }^{t+\Delta t} \mathbb{K} \rightarrow \mathbb{I}^{s}$ when $\Delta \gamma \rightarrow 0$

$$
{ }^{t+\Delta t} \mathbb{D}:={ }^{t+\Delta t} \mathbb{A}_{i n t}^{l e} ;{ }^{t+\Delta t} \mathbb{K}^{-1}
$$

i.e. - note that $\mathbb{D}^{-1}$ (hence, $\mathbb{D}$ ) has major symmetries

$$
\frac{d^{t+\Delta t} \Gamma}{d^{t r} E_{e}}=\frac{{ }^{t+\Delta t} \mathbb{D}:{ }^{t+\Delta t} \nabla \phi_{T}}{{ }^{t+\Delta t} \nabla \phi_{T}:{ }^{t+\Delta t} \mathbb{D}:{ }^{t+\Delta t} \nabla \phi_{T}+\frac{4}{3} \frac{k^{2} k^{\prime}}{3-2 \Gamma k^{j}}} .
$$

Eq. (94) is then

$$
\frac{d^{t+\Delta t} \boldsymbol{E}_{e}}{d^{t r} \boldsymbol{E}_{e}}={ }^{t+\Delta t} \mathbb{K}^{-1}:\left[\mathbb{I}^{s}-\frac{{ }^{t+\Delta t} \nabla \phi_{T} \otimes\left({ }^{t+\Delta t} \mathbb{D}:{ }^{t+\Delta t} \nabla \phi_{T}\right)}{{ }^{t+\Delta t} \nabla \phi_{T}:{ }^{t+\Delta t} \mathbb{D}:{ }^{t+\Delta t} \nabla \phi_{T}+\frac{4}{3} \frac{k^{2} k^{t}}{3-2 \Gamma k^{t}}}\right]
$$

which effectively approaches the fourth-order tensor present in Eq. (57) in the continuum limit, with $\Gamma \rightarrow 0$. Finally, the introduction of this last expression into Eq. ( 90 ) yields

$$
{ }^{t+\Delta^{t}} \mathrm{~A}_{e p}^{\mid t r}=\frac{d^{t+\Delta^{t}} \boldsymbol{T}_{i n t}^{\mid t r}}{d^{t r} \boldsymbol{E}_{e}}={ }^{t+\Delta t} \mathbb{D}-\frac{\left({ }^{t+\Delta t} \mathbb{D}:{ }^{t+\Delta t} \nabla \phi_{T}\right) \otimes\left({ }^{t+\Delta t} \mathbb{D}:{ }^{t+\Delta t} \nabla \phi_{T}\right)}{{ }^{t+\Delta t} \nabla \phi_{T}:{ }^{t+\Delta t} \mathbb{D}:{ }^{t+\Delta t} \nabla \phi_{T}+\frac{4}{3} \frac{k^{2} k^{t}}{3-2 \Gamma k^{t}}}
$$

which clearly represents the algorithmic counterpart of the continuum elastoplastic tangent $\mathbb{A}_{e p}^{l e}$ given in Eq. (59) (just replace the hyperelastic continum moduli $\mathrm{A}_{i n}^{\mid e}$ by the algorithmic moduli $\mathbb{D}$ and take $\Gamma=0$ ) and consistently approaches to it in the continuum limit. Remarkably, the expression given in Eq. (99) adopts an analogous form to that obtained for the equivalent infinitesimal elastoplasticity model [63]. It is also readily seen that the algorithmic elastoplastic tangent moduli ${ }^{t+\Delta t} \mathbb{A}_{c p}^{\mid t r}$ is fully symmetric according to the associative nature of the elastoplastic evolution equation and, ultimately, to the principle of maximum plastic dissipation.

Now, a proper mapping may be performed to the basic stress-strain measures being employed in the finite element program and the selected configuration. As usual in Total Lagrangian finite element codes, we may proceed as in Ref. $[60]$, i.e.

$$
{ }^{t+\Delta t} \mathbb{A}_{e p}^{\mid t r}=\frac{d^{t+\Delta t} \boldsymbol{T}_{i n t}^{\mid t r}}{d^{t r} \boldsymbol{E}_{e}} \rightarrow{ }^{t+\Delta t} \mathbb{C}_{e p}^{\mid t r}=\frac{d^{t+\Delta t} \boldsymbol{S}_{i n t}^{\mid r r}}{d^{t r} \boldsymbol{A}_{e}} \rightarrow{ }^{t+\Delta t} \mathbb{C}_{e p}=\frac{d^{t+\Delta t} \boldsymbol{S}_{i n t}}{d^{t+\Delta t} \boldsymbol{A}}
$$

where we take great advantage from the fact that the trial intermediate configuration remains fixed during global iterations at step $t+\Delta t$, so the final pull-back operation over ${ }^{t+\Delta t} \mathbb{C}_{e p}^{\mid t r}$ to give ${ }^{t+\Delta t} \mathbb{C}_{e p}$ in the reference configuration becomes straightforward, i.e. a mere mapping between fixed, referential configurations, $\mathrm{cf}$. Eq. (102) in Ref. [60]. 
Given ${ }_{0}^{t} \boldsymbol{X}_{p},{ }_{0}^{t+\Delta t} \boldsymbol{X}$ and ${ }_{0}^{t} \gamma$ :

1. Trial elastic logarithmic strains ${ }^{r} \boldsymbol{E}_{e}=\frac{1}{2} \ln \left({ }^{t r} \boldsymbol{X}_{\ell}^{T},{ }^{t r} \boldsymbol{X}_{e}\right)$ with ${ }^{t r} \boldsymbol{X}_{e}={ }^{t+\Delta t}{ }_{0} \boldsymbol{X} \cdot{ }_{0}^{t} \boldsymbol{X}_{p}^{-1}$

2. Associated trial stresses in the fixed intermediate configuration ${ }^{t r} \boldsymbol{T}_{i n t}^{\mid \varepsilon}:=\left.\frac{d \Psi_{i n t}\left(\boldsymbol{E}_{e}\right)}{d \boldsymbol{E}_{e}}\right|_{t r}$

3. In the case ${ }^{t r} f_{T}={ }^{\prime \prime} T_{i n t}^{\mid *}: \mathrm{N}_{T}:{ }^{\prime \prime} T_{j h t}^{\mid *}-\frac{2}{3}{ }^{t r} k^{2}>0$, perform plastic cortection:

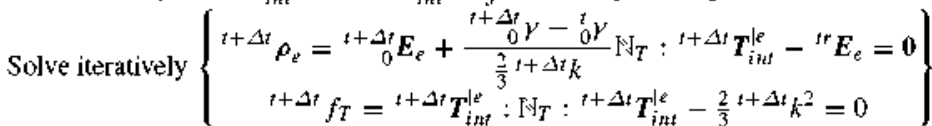

with ${ }^{t+\Delta t} k=k\left({ }_{0}^{t+\Delta t} \gamma\right)$ and initial values ${ }^{t+\frac{\Delta t}{0} E_{\ell}^{(0)}}={ }^{t r} E_{e}$ and ${ }^{t+\Delta t}{ }_{0}^{(0)}={ }^{t} \gamma$.

4. Stresses in fixed intermediate configuration ${ }^{t+\Delta t} \boldsymbol{T}_{i n t}^{\mid t r} \simeq{ }^{t+\Delta t} \boldsymbol{T}_{i n t}^{\left.\right|^{e}}=\left.\frac{d \Psi_{i n t}\left(\boldsymbol{E}_{\ell}\right)}{d \boldsymbol{E}_{e}}\right|_{t+\Delta t}$

5. Map ${ }^{t+\Delta t} \boldsymbol{T}_{i n t}^{\mid t r}$ to second Piola-Kirchhoff stresses ${ }^{t+\Delta t} S_{i n t}^{\mid t r}={ }^{t+\Delta t} \boldsymbol{T}_{i n t}^{\mid r}: \frac{d^{\prime r} E_{\ell}}{d^{t r} \boldsymbol{A}_{\varepsilon}}$

6. Pull back ${ }^{t+\Delta^{t}} S_{i n t}^{j t r}$ to reference configuration ${ }^{t+\Delta t} S_{i n t}={ }_{0}^{t} X_{p}^{-1} \cdot{ }^{t+\Delta t} S_{i n t}^{t r}+{ }_{0}^{t} X_{p}^{-T}$

7. During iterative phase, compute the elasto-plastic tangent ${ }^{t+\Delta_{t}} \mathbb{C}_{k p}$, see Box III

8. During convergence phase, update ${ }^{t+\Delta t} \boldsymbol{X}_{t^{t}}={ }^{t r} \boldsymbol{R}_{t^{*}}+\exp \left({ }^{t+\Delta_{0} t} \boldsymbol{E}_{t^{2}}\right)$

Note: These equations imply ${ }^{t+\Delta^{t}} \boldsymbol{w}_{p}=\mathbf{0}$ as an additional (uncoupled) evolution equation

\section{Box II. Implicit stress integration algorithm.}

Given ${ }^{t+\Delta t} \boldsymbol{E}_{\ell}$ and ${ }^{t+\Delta t} \gamma$;

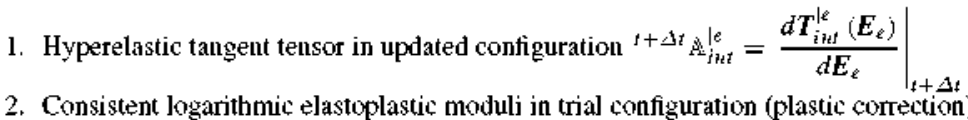

$$
\begin{aligned}
& { }^{t+\Delta t} \mathbb{A}_{\ell^{\prime}}^{\mid t r}=\frac{d^{t+\Delta t} \boldsymbol{T}_{i m t}^{\mid t r}}{d^{t r} E_{e}}={ }^{t+\Delta t} \mathbb{D}-\frac{\left({ }^{t+\Delta t} \mathbb{W j}^{j}:{ }^{t+\Delta t} \nabla \phi_{T}\right) \otimes\left({ }^{t+\Delta t} \mathbb{W}^{j}:{ }^{t+\Delta t} \nabla \phi_{T}\right)}{{ }^{t+\Delta t} \nabla \phi_{T}:{ }^{t+\Delta t} \mathbb{L}^{i}:{ }^{t+\Delta t} \nabla \phi_{T}+\frac{4}{3} \frac{k^{2} k^{\prime}}{3-2 \Gamma_{k}}}
\end{aligned}
$$

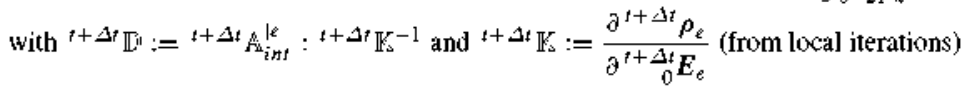

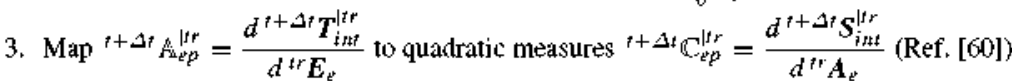

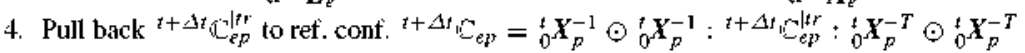

Box III. Consistent elasto-plastic tangent moduli.

The developed numerical algorithm for the internal contribution to stresses and consistent tangent moduli are summarized in the flow-chart diagrams of Boxes II and III.

\section{Model determination from experimental data}

In this section we explain how to fully determine the finite strain elastoplastic model presented above from simple experimental tests. Once the involved stored energy functions and plastic material parameters are identified from experimental data, the model may be used to predict the inelastic response of the characterized material when it is subjected to more general multiaxial non-homogeneous deformation states.

\subsection{Finite strain isotropic compressible material with linear elastoplastic behavior in terms of $\overline{\boldsymbol{\tau}}$ and $\boldsymbol{E}$}

Consider an isotropic elastoplastic material from which we have obtained the following relations from a tensioncompression uniaxial test, performed in direction 1, see Fig. 2

$$
\begin{aligned}
& \left.\begin{array}{c}
\tau_{1}=\Delta \tau_{1}=Y_{e} \Delta E_{1}=Y_{e} E_{1} \\
E_{2}=\Delta E_{2}=-v_{e} \Delta E_{1}=-v_{e} E_{1}
\end{array}\right\} \quad 0 \leq \tau \leq \tau_{y t} \quad \text { Elastic loading } \\
& \left.\begin{array}{c}
\Delta \tau_{1}=Y_{e p} \Delta E_{1} \\
\Delta E_{2}=-v_{e p} \Delta E_{1}
\end{array}\right\} \quad \tau_{y t} \leq \tau \leq \tau_{t r} \quad \text { Elastoplastic loading }
\end{aligned}
$$



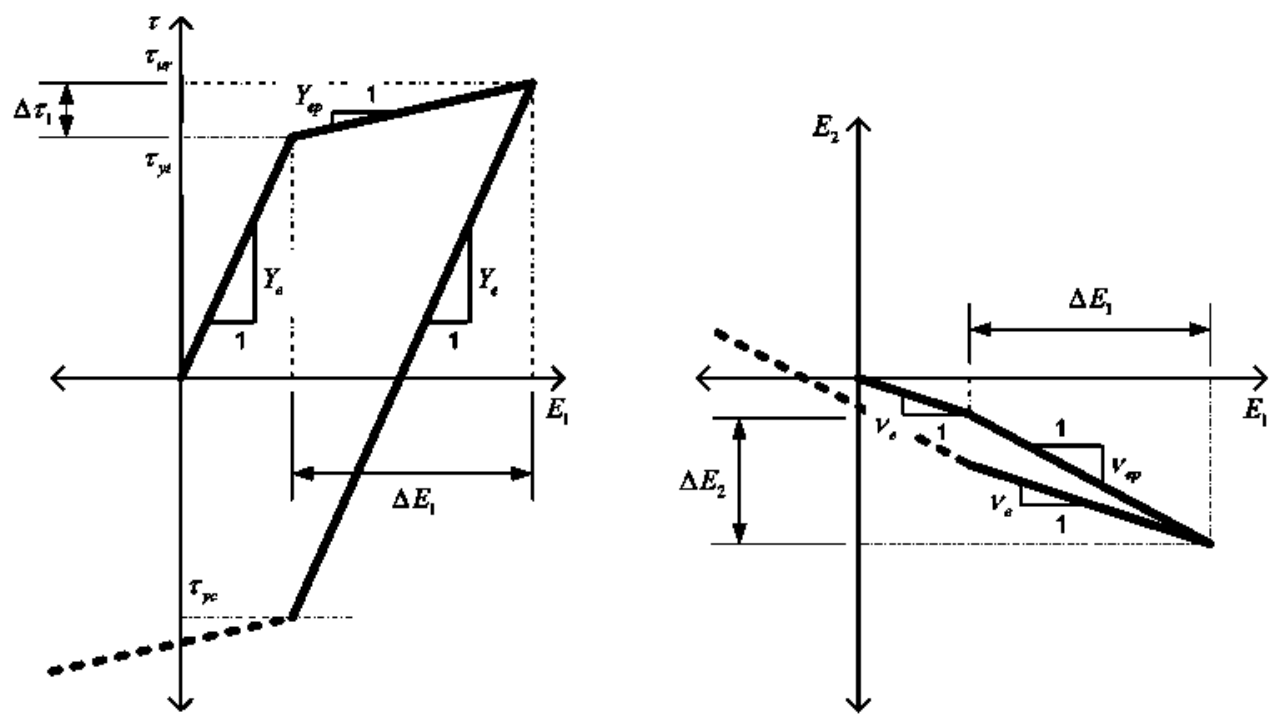

Fig. 2. Representative stress-strain and transverse-to-axial strains responses obtained fron a uniaxial loading-unloading test over an elastoplastic isotropic naterial linear in terns of $\tau$ and $E$. Case with conbined isotropic and kinematic hardening, i.e. with $k^{\prime}>0$ and $2 \mu t_{k i n}>0$.

$$
\left.\begin{array}{c}
\Delta \tau_{1}=Y_{e} \Delta E_{1} \\
\Delta E_{2}=-v_{e} \Delta E_{1}
\end{array}\right\} \quad \tau_{v e} \leq \tau \leq \tau_{u r} \quad \text { Elastic unloading }
$$

where $\tau_{1}, E_{1}$ and $E_{2}$ stand for the macroscopic (measured) uniaxial Kirchhoff stress, uniaxial logarithmic strain and transverse logarithmic strain, respectively. The elastic response is characterized by the Young modulus $Y_{e}$ and the Poisson ratio $v_{e}$, where the subscript $(\cdot)_{e}$ refers to macroscopic "elastic" material constants. The elastoplastic loading starts at the tensile yield point $\tau_{1}=\tau_{y t}>0$ and is characterized by the elastoplastic modulus $Y_{e p}$ and the associated transverse-to-axial strain ratio $v_{e p}$, where the subscript $(\cdot)_{e p}$ refers to macroscopic "elastoplastic" material constants. Finally, an elastic response occurs again between the maximum uniaxial stress reached $\tau_{u r}$ (unloading reference stress) and the plastic-deformation-dependent compressive yield stress $\tau_{1}=\tau_{w_{c}}<0$.

We assume that the internal plastic response is perfectly isochoric. Accordingly, we use Flory's decomposition

$$
\boldsymbol{X}=\boldsymbol{X}^{v} \cdot \boldsymbol{X}^{d}=\left(J^{1 / 3} I\right) \cdot\left(J^{-1 / 3} \boldsymbol{X}\right)
$$

where $J=\operatorname{det} \boldsymbol{X}$ is the volume ratio or Jacobian of the deformation and the superscripts $(\cdot)^{d}$ and $(\cdot)^{v}$ stand for the deviatoric and volumetric parts of the associated tensor variable $(\cdot)$. Then we decompose the truly isochoric deformation gradient using Lee's multiplicative decomposition

$$
\boldsymbol{X}^{d}=\boldsymbol{X}_{e}^{d} \cdot \boldsymbol{X}_{p}^{d}
$$

and propose the following split of the total stored energy $\Psi$ into its volumetric, hyperelastic, part $\mathcal{U}$ and deviatoric, elastoplastic, part $\mathcal{W}=\mathcal{W}_{k i n}+\mathcal{W}_{i n t}$

$$
\Psi\left(\boldsymbol{E}, \boldsymbol{E}_{e}\right)=\mathcal{U}\left(\boldsymbol{E}^{\prime \prime}\right)+\mathcal{W}\left(\boldsymbol{E}^{d}, \boldsymbol{E}_{e}^{d}\right)=\mathcal{U}\left(\boldsymbol{E}^{v}\right)+\mathcal{W}_{k i n}\left(\boldsymbol{E}^{d}\right)+\mathcal{W}_{i n t}\left(\boldsymbol{E}_{e}^{d}\right)
$$

so the deviatoric nature of the internal inelastic response is explicitly enforced by construction [60].

Since the "observed" isotropic behavior is fully linear in terms of the conjugate stress-strain measures pair $\{\overline{\boldsymbol{\tau}}, E\} \equiv\{\boldsymbol{T}, E\}$, where $\overline{\boldsymbol{\tau}}$ are the rotated Kirchhoff stresses [52], the stored energy contributions are quadratic in terms of the respective arguments, i.e.

$$
\begin{aligned}
\Psi\left(\boldsymbol{E}, \boldsymbol{E}_{e}\right) & =\frac{1}{2} \kappa\left(\boldsymbol{t r} \boldsymbol{E}^{v}\right)^{2}+\mu_{k i n}\left\|\boldsymbol{E}^{d}\right\|^{2}+\mu_{i n t}\left\|\boldsymbol{E}_{e}^{d}\right\|^{2} \\
& =\frac{1}{2} \kappa(\ln J)^{2}+\mu_{k i n} \boldsymbol{E}^{d}: \boldsymbol{E}^{d}+\mu_{i n t} \boldsymbol{E}_{e}^{d}: \boldsymbol{E}_{e}^{d}
\end{aligned}
$$

where $\mu_{k i n}$ and $\mu_{i n t}$ are the respective deviatoric, shear moduli (Lame's constants) and $\kappa$ is the (external, unique) bulk modulus. The operation $\|\cdot\|^{2}=(\cdot)$ : (.) stands for the squared standard two norm. The stresses that directly derive 
from Eq. (106) are obtained through Eq. (37), which are purely deviatoric herein, along with the additional volumetric stress $\overline{\boldsymbol{\tau}}^{v}=\boldsymbol{T}^{v}=d \mathcal{U}\left(\boldsymbol{E}^{v}\right) / d \boldsymbol{E}$

$$
\begin{aligned}
\overline{\boldsymbol{\tau}} & =\kappa \ln J \boldsymbol{I}+2 \mu_{k i n} \boldsymbol{E}^{d}: \mathbb{P}^{S}+2 \mu_{i n t} \boldsymbol{E}_{e}^{d}: \mathbb{P}^{S}: \mathbb{I}^{S} \\
& =\kappa \ln J \boldsymbol{I}+2 \mu_{k i n} \boldsymbol{E}^{d}+2 \mu_{i n t} \boldsymbol{E}_{e}^{d} \\
& =\overline{\boldsymbol{\tau}}^{v}+\overline{\boldsymbol{\tau}}_{k i n}^{d}+\overline{\boldsymbol{\tau}}_{i n t}^{d}
\end{aligned}
$$

where $\boldsymbol{I}=d(\ln J) / d \boldsymbol{E}$ is the second-order identity tensor and $\mathbb{P}^{S}=d \boldsymbol{E}^{d} / d \boldsymbol{E}=d \boldsymbol{E}_{e}^{d} / d \boldsymbol{E}_{e}$ is the symmetric fourthorder deviatoric projector tensor. In addition, we have taken into consideration that the fourth-order partial gradient tensor $\partial \boldsymbol{E}_{e} / \partial \boldsymbol{E}_{\dot{X}_{p}=0}$ specializes for this simple uniaxial rotationless case to the symmetric projection tensor, i.e.

$$
\left.\frac{\partial \boldsymbol{E}_{e}}{\partial \boldsymbol{E}}\right|_{\dot{\boldsymbol{X}}_{p}=\mathbf{0}}=\left.\frac{\partial \boldsymbol{E}_{e}^{d}}{\partial \boldsymbol{E}^{d}}\right|_{\dot{X}_{p}=\mathbf{0}}=\left.\frac{\partial\left(\boldsymbol{E}^{d}-\boldsymbol{E}_{p}^{d}\right)}{\partial \boldsymbol{E}^{d}}\right|_{\dot{X}_{p}=\mathbf{0}}=\frac{d \boldsymbol{E}^{d}}{d \boldsymbol{E}^{d}}=\mathbb{I}^{S} .
$$

The incremental Kirchhoff stress tensor and the volumetric-deviatoric decompositions are respectively — since no rotations are present $\boldsymbol{\tau}=\overline{\boldsymbol{\tau}}$

$$
\Delta \boldsymbol{\tau}=\operatorname{diag}\{1,0,0\} \Delta \tau_{1}, \Delta \boldsymbol{\tau}^{d}=\operatorname{diag}\{2,-1,-1\} \frac{\Delta \tau_{1}}{3}, \Delta \boldsymbol{\tau}^{v}=\frac{\Delta \tau_{1}}{3} \boldsymbol{I}
$$

with $\Delta \tau_{1}=Y_{\sharp} \Delta E_{1}$ and where the subscript $(\cdot)_{\#}$ is either $(\cdot)_{e}$ or $(\cdot)_{e p}$, depending on the branch being analyzed. On other hand, the incremental logarithmic strain tensor is

$$
\Delta \boldsymbol{E}=\operatorname{diag}\left\{2,-v_{\#},-v_{\#}\right\} \Delta E_{1}
$$

so

$$
\Delta \boldsymbol{E}^{d}=\operatorname{diag}\{2,-1,-1\} \frac{\left(1+v_{\#}\right) \Delta E_{1}}{3}, \quad \Delta \boldsymbol{E}^{v}=\frac{\left(1-2 v_{\sharp}\right) \Delta E_{1}}{3} \boldsymbol{I} .
$$

Hence, the uniaxial component (axis 1) of Eq. (108), in incremental form, specializes to the following deviatoricvolumetric uncoupled scalar equations

$$
\begin{aligned}
& \Delta \tau_{1}^{d}=\frac{2}{3} \Delta \tau_{1}=\frac{2}{3} Y_{\#} \Delta E_{1}=2 \mu_{k i n} \Delta E_{1}^{d}+2 \mu_{i n t} \Delta E_{e 1}^{d} \\
& \Delta \tau_{1}^{v}=\frac{1}{3} \Delta \tau_{1}=\frac{1}{3} Y_{\#} \Delta E_{1}=\kappa \Delta \ln J=\kappa\left(1-2 v_{\#}\right) \Delta E_{1} .
\end{aligned}
$$

We first deal with Eq. (115), from which we obtain

$$
\kappa=\frac{Y_{\#}}{3\left(1-2 v_{\#}\right)} \text {. }
$$

The particularization of Eq. (116) to both the elastic parameters and the elastoplastic parameters yields a basic relation among the four material constants $Y_{e}, v_{e}, Y_{e p}$ and $v_{e p}$, hence only three of them are independent. Thus, for example, the logarithmic Poisson ratio during elastoplastic loading is given by

$$
\frac{Y_{e p}}{3\left(1-2 v_{e p}\right)}=\frac{Y_{e}}{3\left(1-2 v_{e}\right)} \Rightarrow v_{e p}=\frac{1}{2}-\frac{Y_{e p}}{Y_{e}} \frac{1-2 v_{e}}{2}
$$

which is an expression that we will verify in the first example below.

We focus now on the deviatoric equation in the test direction 1, Eq. (114). Define the macroscopic deviatoric modulus, either elastic or elastoplastic, as

$$
2 \mu_{\#}=\frac{Y_{\#}}{1+\nu_{\#}} .
$$

Then, we can rephrase the left-hand side of Eq. (114) making use of the relation obtained above $\Delta E_{1}^{d}=$ $\frac{2}{3}\left(1+v_{\#}\right) \Delta E_{1}$ as

$$
2 \mu_{\#} \Delta E_{1}^{d}=2 \mu_{k i n} \Delta E_{1}^{d}+2 \mu_{i n t} \Delta E_{e 1}^{d} .
$$

Consider now the initial elastic loading case, as described in Eq. (100), for which $\Delta \boldsymbol{E}_{p}^{d}=\mathbf{0}$, i.e. $\Delta \boldsymbol{E}_{e}^{d} \equiv \Delta \boldsymbol{E}^{d}$, so from Eq. (119) we obtain

$$
\frac{Y_{e}}{1+v_{e}}=: 2 \mu_{e}=2 \mu_{k i n}+2 \mu_{i n t} \text {. }
$$


Consider now Eq. (119) particularized to the tensile yield point, with $E_{1}^{d}=E_{e 1}^{d}=E_{y t}^{d}=\frac{2}{3}\left(1+v_{e}\right) E_{y t}$. The macroscopic deviatoric flow stress (left-hand side of Eq. (119)) is $2 \mu_{e} E_{1}^{d}=2 \mu_{e} E_{y t}^{d}=\tau_{y t}^{d}=\frac{2}{3} \tau_{y t}$ and the internal deviatoric flow stress (second addend on the right-hand side of Eq. (119)) is $2 \mu_{i n t} E_{e 1}^{d}=2 \mu_{i n t} E_{y t}^{d}=k_{0}^{d}=\frac{2}{3} k_{0}$, sonote that in this case $\Delta(\cdot) \equiv(\cdot)$

$$
\frac{2}{3} \tau_{y t}=2 \mu_{k i n} E_{y t}^{d}+\frac{2}{3} k_{0} \Rightarrow \frac{\frac{2}{3} \tau_{y t}}{\frac{2}{3} k_{0}}=\frac{2 \mu_{k i n} E_{y t}^{d}}{2 \mu_{i n t} E_{y t}^{d}}+1
$$

i.e. the internal (initial) yield stress parameter $k_{0}=k(\gamma=0)$, associated with the Saint-Venant (friction) element of the deviatoric counterpart of Fig. 1, relates to the measured, macroscopic flow stress $\tau_{y t}$ through

$$
\frac{k_{0}}{\tau_{y t}}=\frac{2 \mu_{i n t}}{2 \mu_{k i n}+2 \mu_{i n t}}
$$

which is the corresponding macro-to-micro stress conversion factor. We subsequently consider the elastoplastic loading case described in Eq. (101), for which $\Delta \boldsymbol{E}_{p}^{d} \neq \boldsymbol{0}$ and $\left.\Delta \boldsymbol{E}_{e}^{d} \equiv \Delta \boldsymbol{E}_{e}^{d}\right|_{\dot{\gamma}>0}$, so from Eq. (119) we obtain

$$
\frac{Y_{e p}}{1+v_{e p}}=: 2 \mu_{e p}=2 \mu_{k i n}+\left.2 \mu_{i n t} \frac{\Delta E_{e 1}^{d}}{\Delta E_{1}^{d}}\right|_{\dot{\gamma}>0} .
$$

The ratio $\Delta E_{e 1}^{d} /\left.\Delta E_{1}^{d}\right|_{\dot{\gamma}>0}$ may be obtained from the following increments

$$
\begin{aligned}
& \left.\Delta E_{1}^{d}\right|_{\dot{\gamma}>0}=\frac{1}{2 \mu_{e p}}\left(\tau_{u r}^{d}-\tau_{y t}^{d}\right)=\frac{2}{3} \frac{1+v_{e p}}{Y_{e p}}\left(\tau_{u r}-\tau_{y t}\right) \\
& \left.\Delta E_{e 1}^{d}\right|_{\dot{\gamma}>0}=\frac{1}{2 \mu_{e}}\left(\frac{\tau_{u r}^{d}-\tau_{y c}^{d}}{2}-\tau_{y t}^{d}\right)=\frac{2}{3} \frac{1+v_{e}}{Y_{e}}\left(\frac{\tau_{u r}-\tau_{y c}}{2}-\tau_{y t}\right)
\end{aligned}
$$

which may be separately calculated from the experimental stress values $\tau_{y t}, \tau_{u r}$ and $\tau_{y c}$ obtained from the stressstrain response curve in Fig. 2. Eq. (125) represents the increment of the deviatoric elastic domain originated by the plastic deformation path of Eq. (101). Finally, the internal consistency (equilibrium) requirement $2 \mu_{i n t} E_{e 1}^{d}=\frac{2}{3} k(\gamma)$ during the elastoplastic response is expressed in incremental form as $\left.2 \mu_{i n t} \Delta E_{e 1}^{d}\right|_{\dot{\gamma}>0}=\frac{2}{3} k^{\prime} \Delta \gamma$, where the (Kirchhoffstress-like) internal hardening is also linear with respect to the increment of the (logarithmic-strain-like) plastic strain $\gamma$, i.e. $k(\gamma)=k_{0}+k^{\prime} \gamma$ (with $\gamma_{0}=0$ and $\dot{\gamma} \geq 0$ ). Since for this uniaxial case we have $\Delta \gamma=\Delta E_{p 1}^{d}=$ $\left.\Delta E_{1}^{d}\right|_{\dot{\gamma}>0}-\left.\Delta E_{e 1}^{d}\right|_{\dot{\gamma}>0}$ (just particularize Eq. (42) to the present case with $-\left.\dot{\boldsymbol{E}}_{e}\right|_{\dot{\boldsymbol{E}}=\mathbf{0}}=\dot{\boldsymbol{E}}_{p}$ and $\mathbb{N}_{T}=\mathbb{P}^{S}$ ), the consistency requirement gives

$$
\left.\frac{\Delta E_{e 1}^{d}}{\Delta E_{1}^{d}}\right|_{j>0}=\frac{\frac{2}{3} k^{\prime}}{2 \mu_{i n t}+\frac{2}{3} k^{\prime}}
$$

which provides the consistent linearization of the elastoplastic response during plastic flow in Eq. (123), compare this last equation with Eq. (120).

Interestingly, in Eq. (123) we can distinguish two independent contributions to the consistent elastoplastic modulus $2 \mu_{e p}$. The first contribution (second addend in Eq. (123)), namely $2 \mu_{i n t} \times \frac{2}{3} k^{\prime} /\left(2 \mu_{i n t}+\frac{2}{3} k^{\prime}\right)$, vanishes if the presence of plastic flow does not change the size of the elastic domain, i.e. if the internal yield stress $k$ is constant or $k^{\prime}=0$, hence it is associated with a purely isotropic hardening. The second contribution (first addend in Eq. (123)), namely the modulus $2 \mu_{\mathrm{kin}}$ of the Hooke element of the deviatoric counterpart of Fig. 1, is the responsible of the hardening for a constant elastic domain size, i.e. for $k^{\prime}=0$, hence moving the center of the macroscopic elastic domain and being then associated with a purely kinematic hardening.

In summary, in order to determine the isotropic compressible model parameters from a uniaxial test in which we can approximate the response as linear in terms of the stress-strain pair $\{\boldsymbol{\tau}, \boldsymbol{E}\}$, we need to calculate the bulk modulus $\kappa$, the deviatoric moduli $2 \mu_{k i n}$ and $2 \mu_{i n t}$, the internal yield stress $k_{0}$ and the first derivative constant value $k^{\prime}$. We can obtain these parameters from Eqs. (116), (120) and (122)-(126), where we need to know from the uniaxial test curves three elastic-elastoplastic constants, e.g. $Y_{e}, v_{e}$ and $Y_{e p}$, the measured tensile yield stress $\tau_{y t}$ and the test-dependent stress values $\tau_{u r}$ and $\tau_{y c}$. 


\subsection{Orthotropic incompressible material with linear elastoplastic behavior in terms of $\boldsymbol{T}$ and $\boldsymbol{E}$}

We consider in this section three different experimental curve sets from which we can determine the model presented in this work specialized to the case of incompressible orthotropic behavior linear in terms of $T$ and $E$. First of all, we derive all the relations involved in the model determination procedure from uniaxial testing under material preferred directions, which we denote as $\left\{a_{1}, a_{2}, a_{3}\right\}$. Then we will particularize this procedure to the different cases, depending on the experimental data available from experimental testing.

We consider the perfect incompressible case, for which the bulk modulus tends to infinite, i.e. $k \rightarrow \infty$, so the total stored energy may be conceptually written using a mixed formulation, with $p$ and $E^{v}=0$ being unconnected, as

$$
\begin{aligned}
\Psi\left(\boldsymbol{E}, \boldsymbol{E}_{e} ; p\right) & =\mathcal{U}\left(\boldsymbol{E}^{v} ; p\right)+\mathcal{W}_{k i n}\left(\boldsymbol{E}^{d}\right)+\mathcal{W}_{i n t}\left(\boldsymbol{E}_{e}^{d}\right) \\
& =\left(p E^{v}-\frac{1}{2} \frac{p^{2}}{\kappa}\right)+\sum_{i, j=1}^{3} \mu_{i j}^{k i n}\left(E_{i j}^{d}\right)^{2}+\sum_{i, j=1}^{3} \mu_{i j}^{i n t}\left(E_{e i j}^{d}\right)^{2}
\end{aligned}
$$

where $\mu_{i j}^{k i n}$ and $\mu_{i j}^{i n t}$ are the respective orthotropic deviatoric moduli defined in preferred axes $X_{p r}=\left\{\boldsymbol{a}_{1}, \boldsymbol{a}_{2}, \boldsymbol{a}_{3}\right\}$ and $p$ is the pressure-like Lagrange multiplier associated with the incompressibility constraint $E^{v}=\operatorname{tr} E=E_{11}+E_{22}+E_{33}=$ 0 , which is to be determined from the test boundary conditions (i.e. not from $E^{v}$ ).

From now on we consider uniaxial loading cases about the material preferred directions, where a total of six axial-type moduli $\mu_{i i}^{k i t}$ and $\mu_{i i}^{i s t}, i=1,2,3$, are involved and have to be determined. The generalized Kirchhoff stresses $T$ (coincident with the Cauchy stresses $\sigma$ in this case) are obtained again through $\mathrm{Eq}$. (37) along with $\sigma^{\prime \prime}=T^{\prime}=d U\left(E^{v} ; p\right) / d E$ - we use the subscript contraction $(i i) \rightarrow(i), i=1,2,3$

$$
\begin{aligned}
\boldsymbol{T} & =\frac{\partial \mathcal{U}\left(E^{v} ; p\right)}{\partial E^{v}}: \frac{d E^{v}}{d \boldsymbol{E}}+\frac{d \mathcal{W}_{k i n}\left(\boldsymbol{E}^{d}\right)}{d \boldsymbol{E}^{d}}: \frac{d \boldsymbol{E}^{d}}{d \boldsymbol{E}}+\frac{d \mathcal{W}_{i n t}\left(\boldsymbol{E}_{e}^{d}\right)}{d \boldsymbol{E}_{e}^{d}}: \frac{d \boldsymbol{E}_{e}^{d}}{d \boldsymbol{E}_{e}}:\left.\frac{\partial \boldsymbol{E}_{e}}{\partial \boldsymbol{E}}\right|_{\boldsymbol{X}_{p}=\mathbf{0}} \\
& =p \boldsymbol{I}+\left(\sum_{i=1}^{3} 2 \mu_{i}^{k i n} E_{i}^{d} \boldsymbol{a}_{i} \otimes \boldsymbol{a}_{i}\right): \mathbb{P}^{S}+\left(\sum_{i=1}^{3} 2 \mu_{i}^{i n t} E_{e i}^{d} \boldsymbol{a}_{i} \otimes \boldsymbol{a}_{i}\right): \mathbb{P}^{S}: \mathbb{I}^{S} \\
& =\boldsymbol{T}^{v}+\boldsymbol{T}_{k i n}^{d}+\boldsymbol{T}_{i n t}^{d}
\end{aligned}
$$

where the result of Eq. (110) has been used again.

Consider now the Hill-type yield criterion of Eq. (45) with the stress tensor $T_{i n t}^{\mid e}$ being deviatoric by strain energy construction

$$
\boldsymbol{T}_{i n t}^{\mid e}=\frac{d W_{i n t}}{d \boldsymbol{E}_{e}}=\frac{d W_{i n t}}{d \boldsymbol{E}_{e}^{d}}: \frac{d \boldsymbol{E}_{e}^{d}}{d \boldsymbol{E}_{e}}=\frac{d W_{i n t}}{d \boldsymbol{E}_{e}^{d}}: \mathbb{P}^{S}
$$

so we may rephrase Eq. (45) as

$$
f_{T}\left(\boldsymbol{T}_{i n t}^{\mid e}, k\right)=\boldsymbol{T}_{i n t}^{\mid e}: \overline{\mathbb{N}}: \boldsymbol{T}_{i s t}^{\mid e}-\frac{2}{3} k^{2}=0 \text { if } \dot{\gamma}>0
$$

where $\overline{\mathbb{N}}$ is a fourth-order "diagonal" tensor (in matrix, Voigt notation in preferred directions) containing the yielding weights associated with the different "directions" and such that $\mathbb{N}_{T}=\mathbb{P}^{S}: \overline{\mathbb{N}}: \mathbb{P}^{S}$. In matrix notation, and only considering the axial-to-axial components, the tensor $\overline{\mathbb{N}}$ adopts the form

$$
[\overline{\mathrm{N}}]_{X_{p r}}=\left[\begin{array}{ccc}
6 a & 0 & 0 \\
0 & 6 b & 0 \\
0 & 0 & 6 c
\end{array}\right]=\left[\begin{array}{ccc}
1 & 0 & 0 \\
0 & 6 b & 0 \\
0 & 0 & 6 c
\end{array}\right]
$$

where $6 a, 6 b$ and $6 c$ are Hill-like yield weight ratios in the different directions (with the factor of 6 introduced for convenience). Without loss of generality, we consider yield weights with respect to the axis 1 , i.e. $6 a=1$. For von Mises $J_{2}$-plasticity, we obtain $6 a=6 b=6 c=1$ and we recover an internal isotropic yield function, for which $\mathbb{N}_{T}=\mathbb{P}^{S}: \mathbb{I}^{S}: \mathbb{P}^{S}=\mathbb{P}^{S}$, as in the previous section. We discuss herein the case for which both $\mu_{i}^{k i n}>0, i=1,2,3$, and $k=k_{0}$ remain constant, i.e. the case with kinematical hardening alone.

Consider first the uniaxial test performed about the preferred direction $a_{1}$ when the yield point $\boldsymbol{T}_{y_{1}}=$ $\sigma_{y 1}=\operatorname{diag}\left\{\sigma_{y 1}, 0,0\right\}$ is reached after the initial elastic loading, i.e. $E_{p}=\mathbf{0}$ and $\boldsymbol{E}_{e}=\boldsymbol{E}$. We can decompose the 
total yield stress $\sigma_{y 1}$ into its kinematic-type and internal-type contributions through - cf. Ref. [60]

$$
\begin{aligned}
\sigma_{y 1} & =Y_{1}^{e} E_{y 1}=\left(2 \mu_{1}^{e}+\mu_{2}^{e} v_{12}^{e}+\mu_{3}^{e} v_{13}^{e}\right) E_{y 1} \\
& =\left(2 \mu_{1}^{k i n}+\mu_{2}^{k i n} v_{12}^{e}+\mu_{3}^{k i n} v_{13}^{e}\right) E_{y 1}+\left(2 \mu_{1}^{i n t}+\mu_{2}^{i n t} v_{12}^{e}+\mu_{3}^{i n t} v_{13}^{e}\right) E_{y 1} \\
& =Y_{1}^{k i n} E_{y 1}+Y_{1}^{i n t} E_{y 1}=\sigma_{y 1}^{k i n}+\sigma_{y 1}^{i n t} .
\end{aligned}
$$

For further use we define the following internal-to-total flow stress ratios, with $i \neq j \neq k \neq i=\{1,2,3\}$

$$
\rho_{i}:=\frac{\sigma_{y i}^{i n t}}{\sigma_{y i}}=\frac{Y_{i}^{i n t}}{Y_{i}^{e}}=\frac{2 \mu_{i}^{i n t}+\mu_{j}^{i n t} v_{i j}^{e}+\mu_{k}^{i n t} v_{i k}^{e}}{2 \mu_{i}^{e}+\mu_{j}^{e} v_{i j}^{e}+\mu_{k}^{e} v_{i k}^{e}}=\frac{2 \mu_{i}^{i n t} \mu_{j}^{e}+2 \mu_{i}^{i n t} \mu_{k}^{e}+\mu_{j}^{i n t} \mu_{k}^{e}+\mu_{k}^{i n t} \mu_{j}^{e}}{2 \mu_{1}^{e} \mu_{2}^{e}+2 \mu_{2}^{e} \mu_{3}^{e}+2 \mu_{3}^{e} \mu_{1}^{e}}
$$

where we have made use of the general relation for the linear hyperelastic orthotropic model [60]

$$
v_{i j}^{e}=\frac{\mu_{i}^{e}}{\mu_{j}^{e}+\mu_{k}^{e}}
$$

For isotropic behavior $\rho=\mu^{i n t} / \mu^{e}=\mu^{i n t} /\left(\mu^{i n t}+\mu^{k i n t}\right)$. Considering that both $\boldsymbol{E}_{e}^{d}=\boldsymbol{E}_{e}=\boldsymbol{E}_{y 1}=\operatorname{diag}\{\mathbf{1}$, $\left.-v_{12}^{e},-v_{13}^{e}\right\} E_{y 1}$ and $\sigma_{y 1}^{i n t}=Y_{1}^{i n t} E_{y 1}$ at the yield point, the axial-to-axial components of the deviatoric stress tensor $T_{i n t}^{l e}$ of $\mathrm{Eq} .(132)$ particularize to

$$
\left[\boldsymbol{T}_{i n t}^{\mid e}\right]_{X_{p r}}=\left[\begin{array}{c}
\frac{2}{3} \\
-\frac{1}{3} m_{1} \\
-\frac{1}{3}\left(2-m_{1}\right)
\end{array}\right] \sigma_{y, 1}^{i n t}=\left[\begin{array}{c}
\frac{2}{3} \\
-\frac{1}{3} m_{1} \\
-\frac{1}{3}\left(2-m_{1}\right)
\end{array}\right] \rho_{1} \sigma_{y 1}
$$

where we define the following material constant ratios, with $i \neq j \neq k \neq i=\{1,2,3\}$

$$
m_{i}:=\frac{2 \mu_{i}^{i n t}+4 \mu_{j}^{i n t} v_{i j}^{e}-2 \mu_{k}^{i n t} v_{i k}^{e}}{2 \mu_{i}^{i n t}+\mu_{j}^{i n t} v_{i j}^{e}+\mu_{k}^{i n t} v_{i k}^{e}}=\frac{2 \mu_{i}^{i n t} \mu_{j}^{e}+2 \mu_{i}^{i n t} \mu_{k}^{e}+4 \mu_{j}^{i n t} \mu_{k}^{e}-2 \mu_{k}^{i n t} \mu_{j}^{e}}{2 \mu_{i}^{i n t} \mu_{j}^{e}+2 \mu_{i}^{i n t} \mu_{k}^{e}+\mu_{j}^{i n t} \mu_{k}^{e}+\mu_{k}^{i n t} \mu_{j}^{e}} .
$$

For isotropic behavior we obtain a single ratio $m=1$ and $\boldsymbol{T}_{i n t}^{\mid e}=\operatorname{diag}\left\{\frac{2}{3},-\frac{1}{3},-\frac{1}{3}\right\} \sigma_{v}^{i n t}$ at the yield point. The yield function of Eq. (133) vanishes when $\dot{\gamma}>0$

$$
f\left(\boldsymbol{T}_{i n t}^{e}, k\right)=\rho_{1}^{2} \sigma_{y 1}^{2}\left[\begin{array}{c}
\frac{2}{3} \\
-\frac{1}{3} m_{1} \\
-\frac{1}{3}\left(2-m_{1}\right)
\end{array}\right]^{T}\left[\begin{array}{ccc}
6 a & 0 & 0 \\
0 & 6 b & 0 \\
0 & 0 & 6 c
\end{array}\right]\left[\begin{array}{c}
\frac{2}{3} \\
-\frac{1}{3} m_{1} \\
-\frac{1}{3}\left(2-m_{1}\right)
\end{array}\right]-\frac{2}{3} k^{2}=0
$$

which gives an equation relating all the involved deviatoric moduli (through $m_{1}$ and $\rho_{1}$ ), the yield tensor axial parameters $b$ and $c$, the reference internal flow stress $k$ and the measured yield stress $\sigma_{y 1}$ in axis $\mathbf{1}$, i.e.

$$
4 a+m_{1}^{2} b+\left(2-m_{1}\right)^{2} c=\frac{k^{2}}{\left(\sigma_{y 1}^{i n t}\right)^{2}}=\frac{k^{2}}{\rho_{1}^{2} \sigma_{y 1}^{2}} .
$$

The particularization to isotropic behavior yields $6 a=1=k^{2} /\left(\sigma_{y}^{i n t}\right)^{2}$, i.e. $k^{2}=\left(\sigma_{y}^{i n t}\right)^{2}$. Since we are not considering isotropic hardening, then $k=k_{0}$ and $\left(\sigma_{y 1}^{i n t}\right)^{2}$ remains constant whenever $\dot{\gamma}>0$. As a result, the internal stresses of $\mathrm{Eq}$. (140) also remain constant during each elastoplastic uniaxial loading process, with $\sigma_{y 1}^{i n t}>0$ for tension loading and $\sigma_{v 1}^{i t s t}<0$ for compression loading, and the internal elastic strains $\boldsymbol{E}_{e}=\boldsymbol{E}_{y 1}$ remain constant as well, i.e. $\dot{\boldsymbol{E}}_{e}=0$ when $\dot{\gamma}>0$. Hence $\left.\dot{\boldsymbol{E}}\right|_{\dot{\gamma}>0}=\dot{\boldsymbol{E}}_{p}$ when plastic flow is taking place, see Fig. 1.

It is straightforward to obtain

$$
\left(2-m_{2}\right)^{2} a+4 b+m_{2}^{2} c=\frac{k^{2}}{\left(\sigma_{y 2}^{i n t}\right)^{2}}=\frac{k^{2}}{\rho_{2}^{2} \sigma_{y 2}^{2}}
$$




$$
m_{3}^{2} a+\left(2-m_{3}\right)^{2} b+4 c=\frac{k^{2}}{\left(\sigma_{y 3}^{i n t}\right)^{2}}=\frac{k^{2}}{\rho_{3}^{2} \sigma_{y 3}^{2}}
$$

where $\sigma_{y 2}$ and $\sigma_{y 3}$ are the respective uniaxial flow stresses.

Consider now the flow rule of Eq. (42) particularized to the uniaxial case in direction $\boldsymbol{a}_{1}$, along with $-\left.\dot{\boldsymbol{E}}_{e}\right|_{\dot{\boldsymbol{E}}=\mathbf{0}}=\dot{\boldsymbol{E}}_{p}$, i.e.

$$
\left[\dot{\boldsymbol{E}}_{p}\right]_{X_{p r}}=\dot{\gamma} \frac{\sigma_{y 1}^{i n t}}{k}\left[\begin{array}{c}
4 a+m_{1} b+\left(2-m_{1}\right) c \\
-2 a-2 m_{1} b+\left(2-m_{1}\right) c \\
-2 a+m_{1} b-2\left(2-m_{1}\right) c
\end{array}\right] .
$$

Since $\left.\dot{\boldsymbol{E}}\right|_{\dot{\gamma}>0}=\dot{\boldsymbol{E}}_{p}$, the associated logarithmic-strain-based Poisson ratio $v_{12}^{e p}$ during plastic flow becomes

$$
v_{12}^{e p}=-\left.\frac{\dot{E}_{2}}{\dot{E}_{1}}\right|_{\dot{\gamma}>0}=\frac{2 a+2 m_{1} b-\left(2-m_{1}\right) c}{4 a+m_{1} b+\left(2-m_{1}\right) c}=\frac{\mu_{3}^{e p}}{\mu_{2}^{e p}+\mu_{3}^{e p}} .
$$

Due to the fact that $\dot{\boldsymbol{E}}_{e}=\mathbf{0}$ when $\dot{\gamma}>0$, only the external deviatoric moduli govern the incremental elastoplastic response in Eq. (130), i.e. $\mu_{1}^{e p}=\mu_{1}^{k i n}, \mu_{2}^{e p}=\mu_{2}^{k i n}$ and $\mu_{3}^{e p}=\mu_{3}^{k i n}$, which effectively correspond to a purely kinematic hardening case, as discussed in the previous section. Hence we obtain an additional flow compatibility equation in axis 1 relating the involved deviatoric moduli (through $m_{1}$ ) and the yield tensor axial parameters $a, b$ and $c$

$$
2\left(\mu_{2}^{k i n}-\mu_{3}^{k i n}\right) a+\left(2 \mu_{2}^{k i n}+\mu_{3}^{k i n}\right) m_{1} b-\left(\mu_{2}^{k i n}+2 \mu_{3}^{k i n}\right)\left(2-m_{1}\right) c=0 .
$$

This compatibility equation is automatically fulfilled in the isotropic case, so it has not been considered in the foregoing Section. Indeed, Eq. (146) specialized to isotropy simplifies to

$$
\left[\dot{\boldsymbol{E}}_{p}\right]=\frac{\sigma_{y}^{i n t}}{k}\left[\begin{array}{c}
\dot{\gamma} \\
-\dot{\gamma} / 2 \\
-\dot{\gamma} / 2
\end{array}\right] \Rightarrow\left|\Delta E_{p 1}\right|=\Delta \gamma .
$$

The homologous equation to Eq. (148) associated with the uniaxial test about axis 2 reads - the axis - 3 equation is linearly dependent

$$
-\left(\mu_{3}^{k i n}+2 \mu_{1}^{k i n}\right)\left(2-m_{2}\right) a+2\left(\mu_{3}^{k i n}-\mu_{1}^{k i n}\right) b+\left(2 \mu_{3}^{k i n}+\mu_{1}^{k i n}\right) m_{2} c=0
$$

Since we have taken $a=1 / 6$, Eqs. (148) and (150) determine the values of $b$ and $c$ present in the tensor $\overline{\mathbb{N}}$ if all the axial deviatoric moduli (external and internal) are known.

The three Eqs. (143)-(145) along with the two flow rule compatibility constraint Eqs. (148) and (150) define the number of experimental data needed to characterize the elastoplastic orthotropic model under axial loadings about material preferred directions. We discuss next two possible cases, among many others.

\subsubsection{Case (a): One yield stress and both elastic and elastoplastic moduli are known}

Assume that we know the three elastic Young moduli $Y_{1}^{e}, Y_{2}^{e}$ and $Y_{3}^{e}$ (or equivalently two Young moduli $Y_{1}^{e}$ and $Y_{2}^{e}$ and the Poisson ratio $v_{12}^{e}$ ), the three elastoplastic moduli $Y_{1}^{e p}, Y_{2}^{e p}$ and $Y_{3}^{e p}$ (or $Y_{1}^{e p}, Y_{2}^{e p}$ and $v_{12}^{e p}$ ) and also the uniaxial yield stress $\sigma_{y 1}$ in direction $a_{1}$. Then, from the relations (consider also Eq. (139) and its homologous for elastoplastic behavior) - consider $i \neq j \neq k \neq i=\{1,2,3\}$

$$
\begin{aligned}
Y_{i}^{e} & =2 \mu_{i}^{e}+\mu_{j}^{e} v_{i j}^{e}+\mu_{k}^{e} v_{i k}^{e}=\frac{2 \mu_{1}^{e} \mu_{2}^{e}+2 \mu_{2}^{e} \mu_{3}^{e}+2 \mu_{3}^{e} \mu_{1}^{e}}{\mu_{j}^{e}+\mu_{k}^{e}} \\
Y_{i}^{e p} & =2 \mu_{i}^{e p}+\mu_{j}^{e p} v_{i j}^{e p}+\mu_{k}^{e p} v_{i k}^{e p}=\frac{2 \mu_{1}^{e p} \mu_{2}^{e p}+2 \mu_{2}^{e p} \mu_{3}^{e p}+2 \mu_{3}^{e p} \mu_{1}^{e p}}{\mu_{j}^{e p}+\mu_{k}^{e p}}
\end{aligned}
$$

we can obtain the three elastic deviatoric moduli $\mu_{i}^{e}$ and the three elastoplastic deviatoric moduli $\mu_{i}^{e p}$ in any case. We refer to Ref. [67] for admissible values of the respective (whether elastic or elastoplastic) macroscopic moduli $Y_{i}^{\#}$. Since $\mu_{i}^{e}=\mu_{i}^{k i n}+\mu_{i}^{i n t}$ and $\mu_{i}^{e p}=\mu_{i}^{k i n}$, we have the six axial moduli $\mu_{i}^{k i n}$ and $\mu_{i}^{i n t}$ present in the stresses of Eq. (130). Then we know the three ratios $\rho_{i}$ and the three ratios $m_{i}$ defined in Eqs. (138) and (141), respectively, and we can solve the two flow rule compatibility equations (148) and (150) in order to obtain the yielding tensor weights $b$ and $c$ in Eq. (134). 
Finally, in order to completely define our model in material preferred directions, we need the internal yield stress parameter $k$, which can be directly obtained from Eq. (143) using the measured yield stress $\sigma_{y 1}$.

\subsubsection{Case (b): One elastoplastic modulus and both elastic moduli and yield stresses are known}

Assume that, from the uniaxial testing response over preferred directions of an incompressible orthotropic material, we know the three elastic Young moduli $Y_{1}^{e}, Y_{2}^{e}$ and $Y_{3}^{e}$ (or $Y_{1}^{e}, Y_{2}^{e}$ and $v_{12}^{e}$ ), the three uniaxial yield stresses $\sigma_{y 1}, \sigma_{y 2}$ and $\sigma_{y 3}$ and also the elastoplastic modulus $Y_{1}^{e p}$. Unlike the preceding case (a), we can obtain the three elastic deviatoric moduli $\mu_{i}^{e}\left(=\mu_{i}^{k i n}+\mu_{i}^{i n t}\right)$ but we can obtain neither the three elastoplastic deviatoric moduli $\mu_{i}^{e p}\left(=\mu_{i}^{k i n}\right)$ nor the ratios $\rho_{i}$ and $m_{i}$.

In this case, we have to solve the complete system of five nonlinear equations detailed above, i.e. Eqs. (143)-(145), (148) and (150), along with Eq. (152) for $i=1$ with the modulus $Y_{1}^{e p}$ being known. From these six equations, with $\rho_{i}$ and $m_{i}$ defined in Eqs. (138) and (141) and taking $\mu_{i}^{i n t}=\mu_{i}^{e}-\mu_{i}^{k i n}$, we can obtain the involved unknowns of the elastoplastic model under consideration, namely the internal yield function parameter $k$, the yield tensor weights $b$ and $c$ and the deviatoric moduli $\mu_{1}^{k i n}, \mu_{2}^{k i n}$ and $\mu_{3}^{k i n}$. Finally, the remaining three internal deviatoric moduli are calculated by means of $\mu_{i}^{i n t}=\mu_{i}^{e}-\mu_{i}^{k i n}$.

\section{Examples}

We first perform in this section some examples in order to verify that the determination procedures for the internal ("microscopic") parameters detailed just above for our model are consistent with the observed ("macroscopic") responses obtained from homogeneous uniaxial simulations. Once this is verified for both isotropic and orthotropic materials, the capabilities of the present computational formulation are shown through additional non-homogeneous finite element simulations.

\subsection{Isotropic compressible material with linear elastoplastic behavior in terms of $\overline{\boldsymbol{\tau}}$ and $\boldsymbol{E}$}

We have explained in Section 6.1 how we can characterize an isotropic compressible material which shows an elastoplastic response that may be considered linear in terms of the work-conjugate pair of rotated Kirchhoff stresses and logarithmic strains as a first approximation $[68,69]$. In this case the model parameters to be determined are, on one hand, the bulk modulus $\kappa$ and the deviatoric moduli $2 \mu_{k i n}$ and $2 \mu_{i n t}$ (needed to define the total stored energy of Eq. (106)) and, on the other hand, the (initial) internal yield stress $k_{0}$ and the first derivative constant value $k^{\prime}$ (needed to define the evolution of the internal yield parameter $k=k_{0}+k^{\prime} \gamma$ ). We have shown above that these model parameters can be obtained from a loading-unloading uniaxial test in which we measure the Young modulus $Y_{e}$ and the Poisson ratio $v_{e}$ during the initial elastic loading, the tensile yield stress $\tau_{y t}$, the elastoplastic modulus $Y_{e p}$ and the maximum stress value (unloading reference) $\tau_{u r}$ during the plastic loading and, finally, the compressive yield stress $\tau_{y c}$ upon the elastic unloading.

Assume that we have measured the following experimental values from a loading-unloading uniaxial test over a soft isotropic compressible material, see Eqs. (100)-(102) and Fig. 2

$$
\begin{array}{rlrl}
Y_{e} & =24 \mathrm{MPa}, & v_{e}=0.2, \quad Y_{e p}=24 / 5=4.8 \mathrm{MPa} \\
\tau_{y t} & =12 \mathrm{MPa}, & & \tau_{u r}=15 \mathrm{MPa}, \quad \tau_{y c}=-12 \mathrm{MPa} .
\end{array}
$$

The associated elastoplastic Poisson ratio, as defined in Eq. (101), is obtained from Eq. (117) and takes the value $v_{e p}=0.44$. The corresponding bulk modulus in Eq. (106) is obtained from Eq. (116), taking the value

$$
\kappa=\frac{Y_{e}}{3\left(1-2 v_{e}\right)}=\frac{Y_{e p}}{3\left(1-2 v_{e p}\right)}=40 / 3=13.33 \mathrm{MPa} .
$$

The direct evaluation of Eqs. (124) and (125) gives the deviatoric strain increments $\left.\Delta E_{1}^{d}\right|_{\dot{\gamma}>0}=0.6$ and $\left.\Delta E_{e 1}^{d}\right|_{\dot{\gamma}>0}=$ 0.05 . Then, we can obtain both deviatoric modulus $2 \mu_{k i n}$ and $2 \mu_{i n t}$ solving Eqs. (120) and (123), which yields

$$
2 \mu_{\text {kin }}=20 / 11=1.818 \mathrm{MPa}, \quad 2 \mu_{\text {int }}=200 / 11=18.18 \mathrm{MPa} .
$$



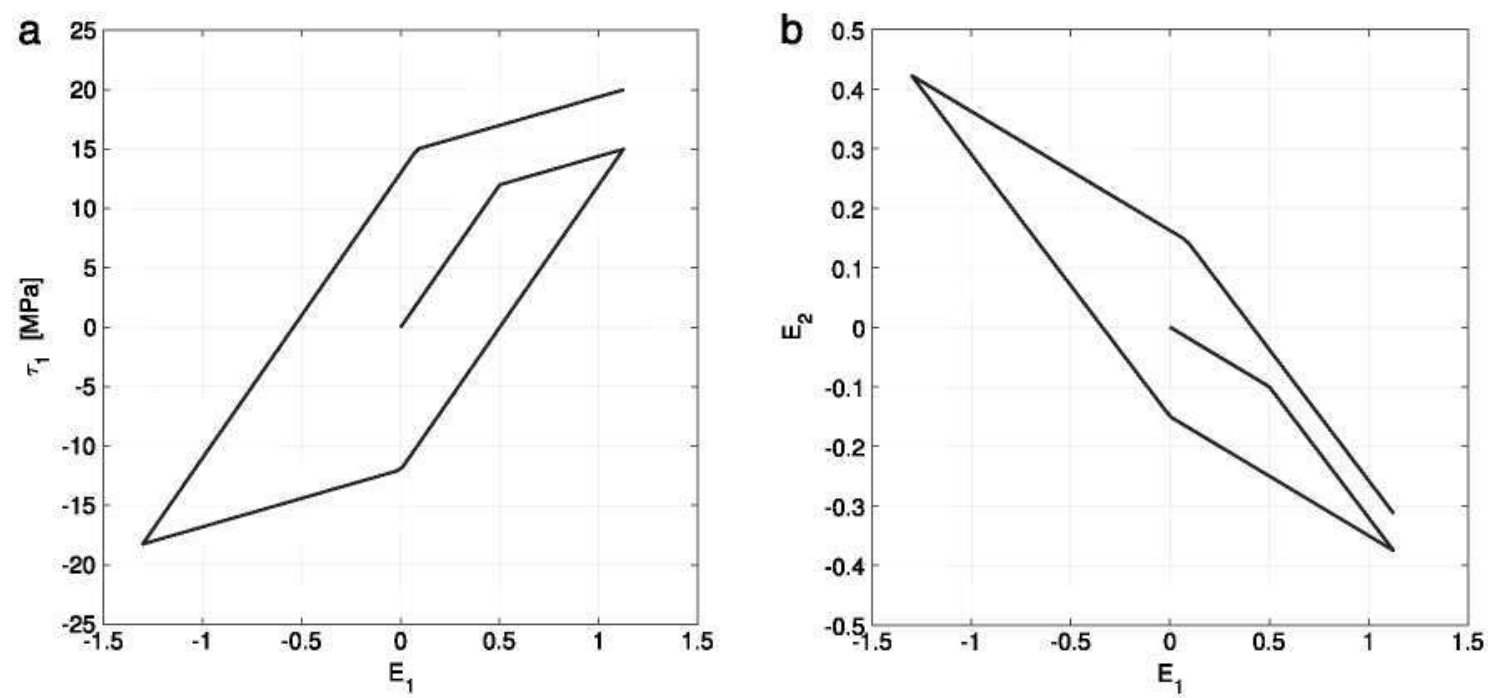

Fig. 3. Uniaxial loading-unloading test. Case with combined isotropic and kinematic hardening, i.e. with $k^{\prime}>0$ and $2 \mu_{k i n}>0$. Left (a) Axial Kirchhoff stresses $\tau_{1}\left(E_{1}\right)$. Right (b) Transverse strains $E_{2}\left(E_{1}\right)$.

Once these values are known, the direct evaluation of Eqs. (122) and (126) gives finally the remaining parameters

$$
k_{0}=120 / 11=10.91 \mathrm{MPa}, \quad k^{\prime}=300 / 121=2.479 \mathrm{MPa} .
$$

We have used the model parameters presented in Eqs. (155)-(158) to define the material model in our in-house Finite Element code Dulcinea, where the general algorithmic formulation of Section 5 has been implemented. The internal yield criterion is the von Mises criterion, with the yield function given in Eq. (45) along with

$$
\boldsymbol{T}_{i n t}^{\mid e}: \mathbb{N}_{T}: \boldsymbol{T}_{i n t}^{\mid e}=\boldsymbol{T}_{i n t}^{\mid e}: \overline{\mathbb{N}}: \boldsymbol{T}_{i n t}^{\mid e}=\boldsymbol{T}_{i n t}^{\mid e}: \mathbb{I}^{S}: \boldsymbol{T}_{i n t}^{\mid e}=\boldsymbol{T}_{i n t}^{\mid e}: \boldsymbol{T}_{i n t}^{\mid e}=\left\|\boldsymbol{T}_{i n t}^{\mid e}\right\|^{2}=\left\|\boldsymbol{\tau}_{i n t}^{\mid e}\right\|^{2} .
$$

We have then simulated a displacement-driven loading-unloading uniaxial test over that material in order to verify that the measured macroscopic moduli and stresses used to define the model are recovered, i.e. those given in Eqs. (153)-(154), hence proving the consistency of both the model determination procedure and the model formulation. Since the deformation is homogeneous at each loading step and the elastoplastic behavior, with $v_{e p}=0.44$, may be regarded as slightly compressible, we have used only one standard Brick element of 8 nodes, i.e. a mixed formulation is not required in this case. We finally note that all the deformations involved in this example (dilatation one and deviatoric elastic and plastic ones) become large and that the finite element global computations are materially (in terms of $\boldsymbol{S}$ and $\boldsymbol{A}$ ) and geometrically nonlinear, as we show below.

We show the results of the computation in Fig. 3. In Fig. 3(a) it can be observed that the modulus of every elastic response is $Y_{e}=24 \mathrm{MPa}$, that the modulus of every elastoplastic response is $Y_{e p}=4.8 \mathrm{MPa}$ and that the (first) yield point effectively occurs at $\tau_{y t}=12 \mathrm{MPa}$. We have applied a first (displacement) load in the test direction such that $E_{*}=1.125$ (i.e. $\left.E_{u r}=\tau_{y t} / Y_{e}+\left(\tau_{u r}-\tau_{y t}\right) / Y_{e p}\right)$. Consistently with this extension load, we can see that the maximum stress during the first elastoplastic loading, namely $\tau_{u r}=15 \mathrm{MPa}$, is retrieved, which moreover provokes the prescribed increment of the macroscopic elastic domain, i.e. an increment such that $\tau_{y c}=-12 \mathrm{MPa}$ upon the first elastic unloading. Note that the initial macroscopic yield surface size is given by $\varsigma_{0}:=\tau_{y t}=12 \mathrm{MPa}$, whereas the increased elastic domain size after the first plastic loading becomes $\varsigma_{1}=\frac{1}{2}\left(\tau_{u r}-\tau_{y c}\right)=13.5 \mathrm{MPa}$. This macroscopic (observed, apparent) increment is associated with an upscaled size of the internal von Mises surface, which is computed from $\varsigma_{1}$ through the macro-to-micro conversion factor - cf. Eq. (122)

$$
k_{1}=\varsigma_{1} \times \frac{2 \mu_{i n t}}{2 \mu_{k i n}+2 \mu_{i n t}}=12.27 \mathrm{MPa}
$$

which is also consistent with the one obtained from the linear internal isotropic hardening law

$$
k_{y c}=k_{0}+k^{\prime} \Delta \gamma=k_{0}+\left.k^{\prime} \Delta E_{p 1}^{d}\right|_{\dot{\gamma}>0}=k_{0}+k^{\prime}\left(\Delta E_{1}^{d}-\Delta E_{e 1}^{d}\right)_{\dot{\gamma}>0}=12.27 \mathrm{MPa} .
$$




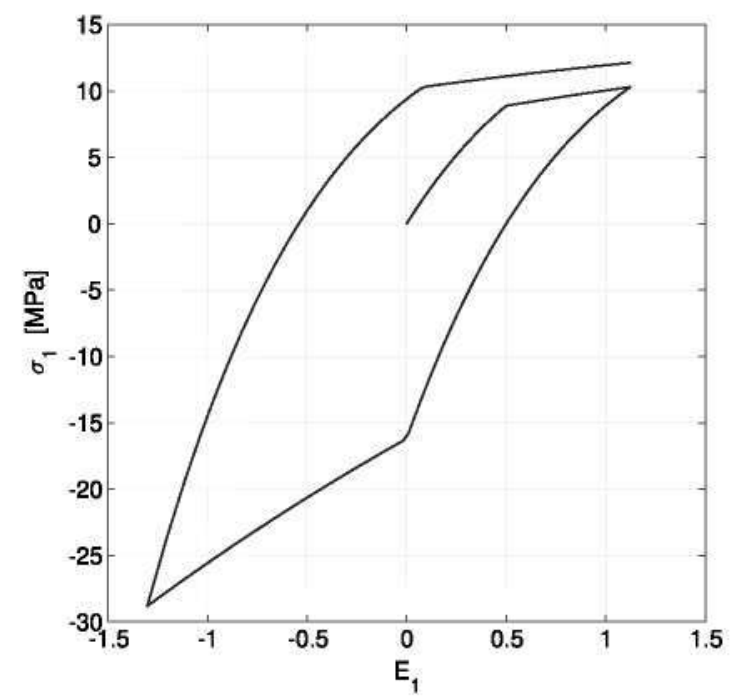

Fig. 4. Uniaxial loading-unloading test. Case with combined isotropic and kinematic hardening, i.e. with $k^{\prime}>0$ and $2 \mu_{k i n}>0$. Axial Cauchy stresses $\sigma_{1}\left(E_{1}\right)$.

Table 1

Asymptotic quadratic convergence: Unbalanced force and energy during a typical elastoplastic step using a global Newton-Raphson scheme.

\begin{tabular}{lll}
\hline Iteration & Force & Energy \\
\hline 1 & $1.000 \mathrm{E}+00$ & $1.000 \mathrm{E}+00$ \\
2 & $1.239 \mathrm{E}-02$ & $1.499 \mathrm{E}-04$ \\
3 & $1.849 \mathrm{E}-06$ & $3.336 \mathrm{E}-12$ \\
4 & $1.100 \mathrm{E}-12$ & $2.440 \mathrm{E}-24$ \\
\hline
\end{tabular}

After the first plastic loading is completed, the new center of the elastic domain for the uniaxial test is located at $c_{1}=\frac{1}{2}\left(\tau_{u r}+\tau_{y c}\right)=1.5 \mathrm{MPa}$, i.e. the hardening is originated by a combination of isotropic (through $\left.k^{\prime}\right)$ and kinematic (through $2 \mu_{k i n}$ ) effects, recall Section 6.1.

It can be seen in Fig. 3(b) that the independent, "experimentally" observed Poisson ratio under elastic loading, namely $v_{e}=0.2$, is also reproduced during every elastic response. Furthermore, the dependent, analytically computed Poisson ratio under plastic loading of Eq. (117), namely $v_{e p}=0.44$, is consistently reproduced by the simulation during every elastoplastic loading. Note that we have not introduced these values explicitly in the finite element material model.

In Fig. 4 we represent the uniaxial response curve in terms of (rotated) Cauchy stresses (work-conjugate stresses, per unit current volume, of logarithmic strains for isotropic solids) in order to notice the non-linearity involved in the problem. Note that the high differences between Kirchhoff and Cauchy stress values (ordinates) are due to the volume ratio at each deformation state, i.e. $\tau_{1}=J \sigma_{1}$, which indicates that very large volume changes are taking place in the uniaxial test. The rates of convergence for residual force and energy during a standard elastoplastic step are asymptotically quadratic, see Table 1 . Similar ratios are obtained for the local Newton iterations.

Finally, just as representative examples of the cases with isotropic hardening only, kinematic hardening only and no hardening, we represent in Figs. 5-7 the uniaxial response curves of the respective (modified) material model when we enforce $k^{\prime}>0$ and $2 \mu_{k i n}=0, k^{\prime}=0$ and $2 \mu_{k i n}>0$ or $k^{\prime}=0$ and $2 \mu_{k i n}=0$ respectively, leaving the remaining model parameters unchanged in each case. Different macroscopic elastic and plastic responses are obtained accordingly. We can observe that in the case with $k^{\prime}>0$ and $2 \mu_{k i n}=0$, Fig. 5 , the center of the elastic domain in the space of $\{\boldsymbol{\tau}, \boldsymbol{E}\}$ always remains at the origin of stresses and that the size $\zeta$ of the elastic domain is increased after each elastoplastic loading, i.e. an isolated isotropic hardening effect. On the other side, in the case with $k^{\prime}=0$ and $2 \mu_{k i n}>0$, Fig. 6 , the center of the elastic domain evolves in parallel to the incremental elastoplastic stresses, i.e. both with slope $2 \mu_{k i n}$ in the space of $\{\boldsymbol{\tau}, \boldsymbol{E}\}$, and the elastic domain preserves its size $\varsigma=\varsigma_{0}$ after each 

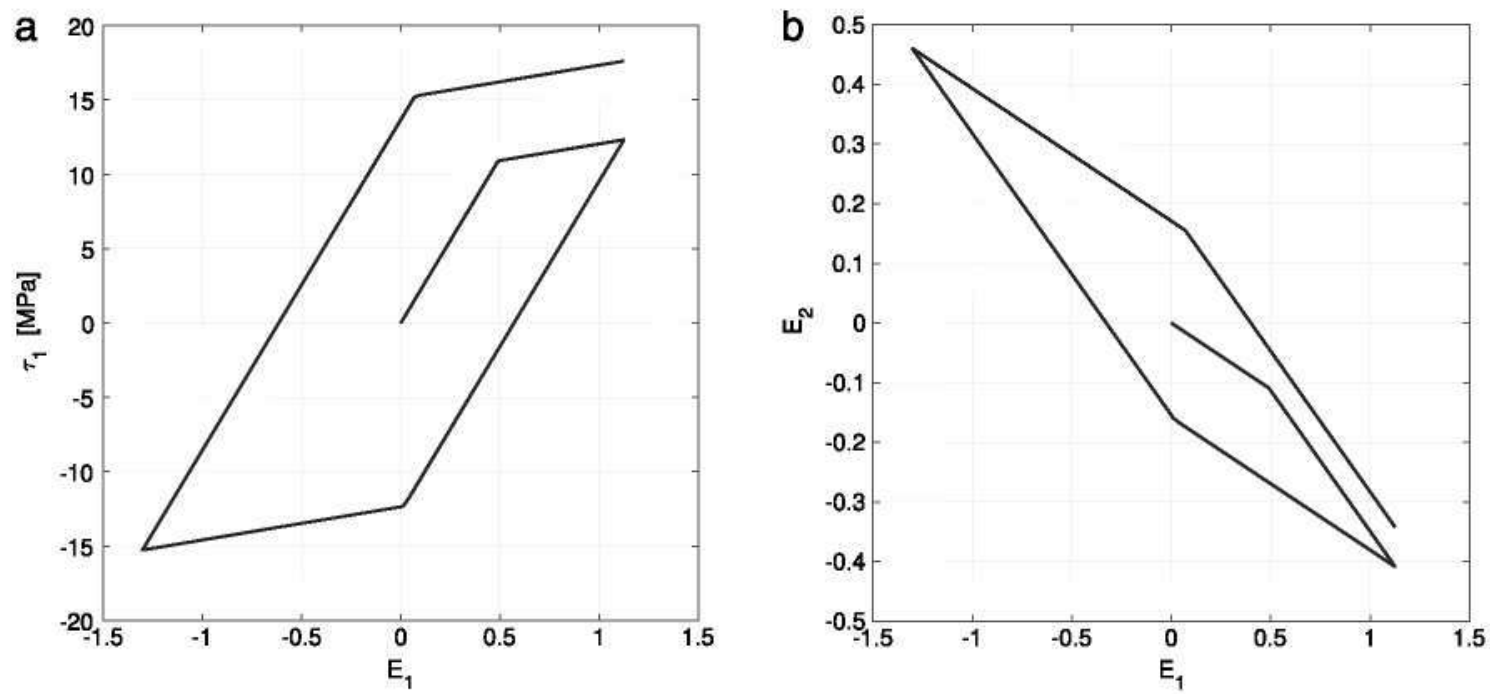

Fig. 5. Uniaxial loading-unloading test. Case with isotropic hardening only, i.e. with $k^{\prime}>0$ and $2 \mu_{k i n}=0$. Left (a) Axial Kirchhoff stresses $\tau_{1}\left(E_{1}\right)$. Right (b) Transverse strains $E_{2}\left(E_{1}\right)$.
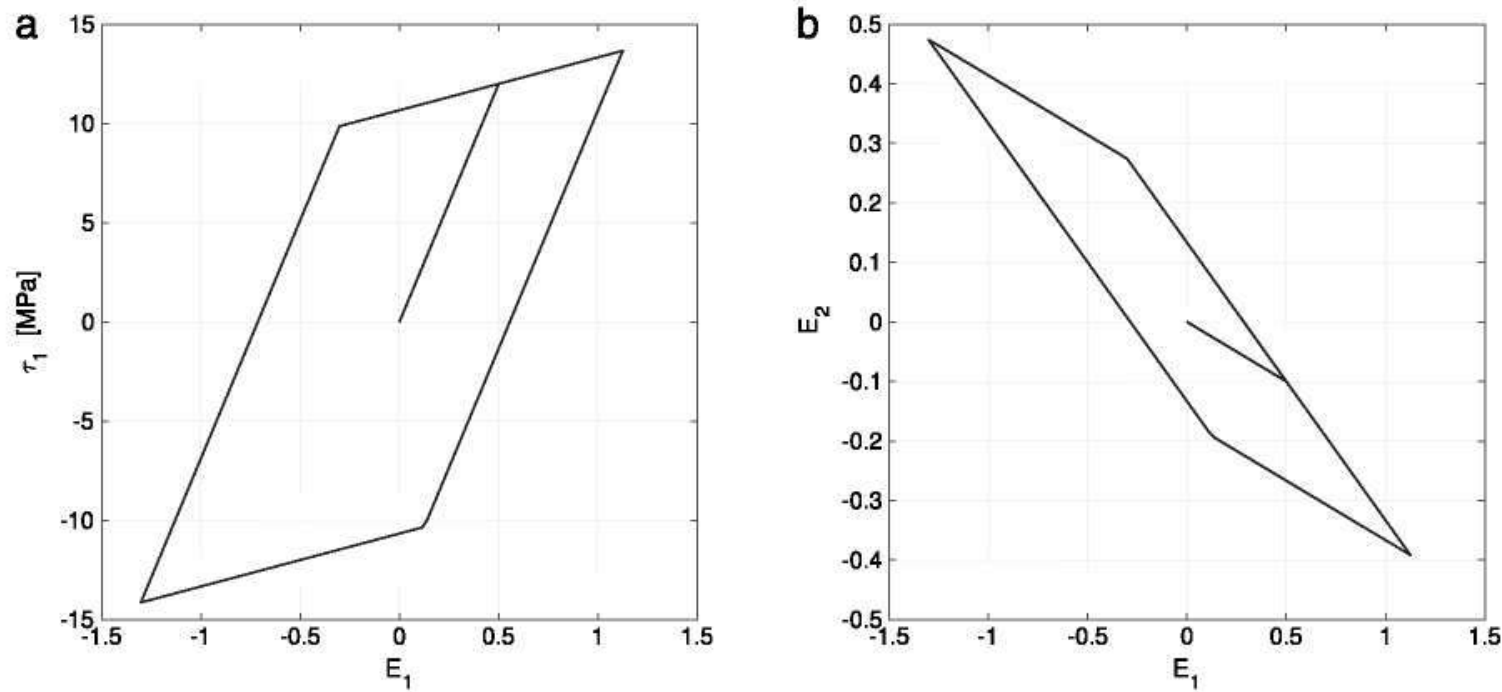

Fig. 6. Uniaxial loading-unloading test. Case with kinematic hardening only, i.e. with $k^{\prime}=0$ and $2 \mu_{k i n}>0$. Left (a) Axial Kirchhoff stresses $\tau_{1}\left(E_{1}\right)$. Right (b) Transverse strains $E_{2}\left(E_{1}\right)$.

elastoplastic loading. The stress-strain and transverse-to-axial strains cycles are closed, i.e. an isolated kinematic hardening (Bauschinger) effect is taking place. Finally, for the case with both $k^{\prime}=0$ and $2 \mu_{k i n}=0$, Fig. 7, a perfect plasticity response is obtained in terms of both Kirchhoff and Cauchy stresses. Note that in this last case, i.e. Fig. 7, $v_{e p}=0.5$ because $Y_{e p}=0$, recall Eq. (117), so $\left.\Delta J\right|_{\dot{\gamma}>0}=0$, i.e. $J$ remains constant during each plastic loading, and $\left.\Delta \sigma_{1}\right|_{\dot{\gamma}>0}=\left.\frac{1}{J} \Delta \tau_{1}\right|_{\dot{\gamma}>0}=0$.

\subsection{Orthotropic incompressible material with linear elastoplastic behavior in terms of $\boldsymbol{T}$ and $\boldsymbol{E}$}

In this example we obtain the material model parameters from an experimental data set as the one addressed in Section 6.2.2. Then we perform three different uniaxial test finite element simulations along the three preferred directions in order to show that the numerical results obtained from the computations are in correspondence with the 

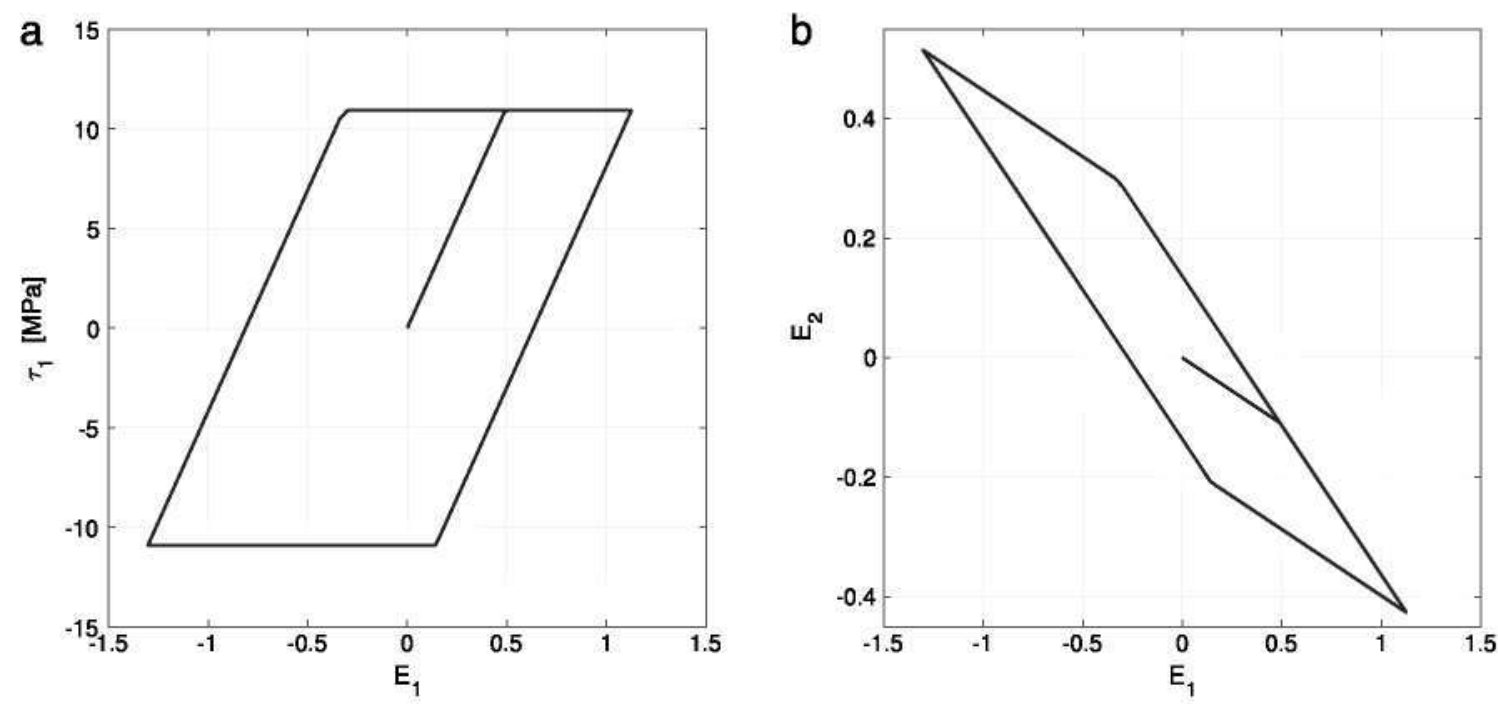

Fig. 7. Uniaxial loading-unloading test. Perfect plasticity case (no hardening), i.e. with $k^{\prime}=0$ and $2 \mu_{k i n}=0$. Left (a) Axial Kirchhoff stresses $\tau_{1}\left(E_{1}\right)$. Right (b) Transverse strains $E_{2}\left(E_{1}\right)$.

prescribed data. We will see that the simulations also reproduce all the remaining theoretical (elastoplastic) moduli and (elastic and elastoplastic) Poisson' ratios introduced above.

Assume that we have measured the following "experimental" values from uniaxial testing about the preferred directions $X_{p r}=\left\{\boldsymbol{a}_{1}, \boldsymbol{a}_{2}, \boldsymbol{a}_{3}\right\}$ of an orthotropic (soft) material with a linear response in terms of $\boldsymbol{T}$ and $\boldsymbol{E}$ under finite strains

$$
\begin{aligned}
& Y_{1}^{e}=18.86 \mathrm{MPa}, \quad Y_{2}^{e}=13.2 \mathrm{MPa}, \quad Y_{3}^{e}=10.15 \mathrm{MPa} \\
& \sigma_{y 1}=4.889 \mathrm{MPa}, \quad \sigma_{y 2}=4.334 \mathrm{MPa}, \quad \sigma_{y 3}=3.933 \mathrm{MPa}
\end{aligned}
$$

and

$$
Y_{1}^{e p}=7.333 \mathrm{MPa}
$$

where $Y_{i}^{e}, \sigma_{y i}$ and $Y_{i}^{e p}$ stand for the elastic Young modulus, the yield stress and the elastoplastic modulus corresponding to the $i$-axis test, respectively. From Eqs. (151), with $Y_{1}^{e}, Y_{2}^{e}$ and $Y_{3}^{e}$ being known, we first obtain

$$
\mu_{1}^{e}=8 \mathrm{MPa}, \quad \mu_{2}^{e}=5 \mathrm{MPa}, \quad \mu_{3}^{e}=2 \mathrm{MPa} .
$$

Then, from Eq. (152) with $Y_{1}^{e p}=7.333 \mathrm{MPa}$ and $\mu_{i}^{e p}=\mu_{i}^{k i n}$ (i.e. an isolated kinematic hardening case), along with Eqs. (143)-(145), (148) and (150), with $\rho_{i}$ and $m_{i}$ defined in Eqs. (138) and (141) and considering $\mu_{i}^{i n t}=\mu_{i}^{e}-\mu_{i}^{k i n}$, we obtain - in order to solve this system of nonlinear equations and take numerical advantage of its symmetries, it is convenient to define the modified yield tensor components $\hat{a}=a / k^{2}, \hat{b}=b / k^{2}, \hat{c}=c / k^{2}$ and then undo this change of variables just considering that $6 a=1$

$$
\begin{aligned}
& \mu_{1}^{k i n}=3 \mathrm{MPa}, \quad \mu_{2}^{k i n}=2 \mathrm{MPa}, \quad \mu_{3}^{k i n}=1 \mathrm{MPa} \\
& 6 b=2.324, \quad 6 c=3.647
\end{aligned}
$$

and

$$
k=k_{0}=3.850 \mathrm{MPa} .
$$

Finally, the three relations $\mu_{i}^{i n t}=\mu_{i}^{e}-\mu_{i}^{k i n}$ yield

$$
\mu_{1}^{i n t}=5 \mathrm{MPa}, \quad \mu_{2}^{i n t}=3 \mathrm{MPa}, \quad \mu_{3}^{i n t}=1 \mathrm{MPa} .
$$


The (axial type) deviatoric moduli given in Eqs. (165) and (168), the (axial type) components of the yield tensor $\overline{\mathbb{N}}$ given in Eq. (166) and the internal flow stress parameter given in Eq. (167) define the material model in orthotropy preferred directions. We have used these numerical values in order to define our computational model. Then, we have separately performed three uniaxial test finite element calculations in the respective preferred axes with the main aim of verifying that the primary experimental data of Eqs. (161)-(163) are reproduced by the simulations. This way we verify the consistency of the material model determination procedure explained in Section 6.2 with the finite element algorithmic formulation presented in this work. We have used in these simulations a single $u / p$ mixed finite element brick $Q 1 / P 0$ (or $8 / 1$ ) because the deformation is homogeneous all over the element. As in the previous isotropic case example, we have used the volumetric strain energy function $\mathcal{U}(J)=\frac{1}{2} \kappa(\ln J)^{2}$, but in this case it is employed just as a penalty function to enforce quasi-incompressibility during the computations, with the bulk modulus taken as $\kappa=10^{4} \mathrm{MPa}$ (i.e. $\mathcal{U}(\mathrm{J})$ replaces the theoretical volumetric function present in Eq. (128) for the matter of computational convenience).

In Fig. 8 we represent the uniaxial stress-strain and transverse-to-axial strains response curves obtained from the computations, which in the three cases $(i=1,2,3)$ have been driven up to a maximum logarithmic strain of $E_{i}=1$ (i.e. maximum extension) and a minimum logarithmic strain of $E_{i}=-1$ (i.e. maximum compression). We can observe in the stress-strain solution curves that the three preferred elastic Young moduli $Y_{i}^{e}$, the three tensile yield stresses $\sigma_{y i}$ and the single preferred elastoplastic modulus $Y_{1}^{e p}$ are in perfect agreement with the prescribed "experimental" values of Eqs. (161)-(163), which have been used to determine the material model parameters of Eqs. (165)-(168). Additionally, we can observe that the two other elastoplastic moduli $Y_{2}^{e p}$ and $Y_{3}^{e p}$ predicted by the simulations are in perfect correspondence with the analytical ones obtained from Eq. (152), i.e. in both cases (response curves and analytical relations) we obtain

$$
Y_{2}^{e p}=5.5 \mathrm{MPa} \text { and } Y_{3}^{e p}=4.4 \mathrm{MPa} .
$$

The computed elastic and elastoplastic (logarithmic-type) Poisson ratios $v_{i j}=-E_{j} / E_{i}$, which can be measured in the transverse-to-axial strains response curves, are also coincident to the respective analytical ones, which can be obtained from Eq. (139) and its elastoplastic homologous one. In both cases, the Poisson ratios obtained are — in this case they are almost coincident for each test

$$
\begin{aligned}
& v_{12}^{e}=0.286=\frac{2}{7}, \quad v_{23}^{e}=0.8=\frac{4}{5}, \quad v_{31}=0.385=\frac{5}{13} \\
& v_{12}^{e p}=0.333=\frac{1}{3}, \quad v_{23}^{e p}=0.75=\frac{3}{4}, \quad v_{31}^{e p}=0.4=\frac{2}{5} .
\end{aligned}
$$

Finally, note that all the solution curves (both stress-strain and transverse-to-axial strains) in Fig. 8 are closed cycles, which is a direct consequence of having considered a constant (internal) yield stress parameter $k=k_{0}$. In particular, the size of the macroscopic elastic domain is preserved in each stress-strain curve and is equal to $\varsigma_{i}=2 \sigma_{y i}$. In other words, the hardening response is purely kinematic (as prescribed) and governed by the external deviatoric moduli given in Eq. (165), cf. Eq. (152) with $\mu_{i}^{e p}=\mu_{i}^{k i n}$. The additional consideration of an internal isotropic hardening law $k(\gamma)$, with $k^{\prime}(\gamma)>0$, would give an internal Hill yield surface (i.e. a six-dimensional ellipsoid in terms of the components of $\boldsymbol{T}_{i n t}^{\mid e}, \mathrm{cf}$. Eq. (45)) with all its "axes" increasing proportionally to $k / k_{0}$, which would correspond with a mixed hardening formulation for the fully orthotropic elastoplastic material under study. A combined hardening effect of this type will be discussed in the last example below including also both elastic and plastic non-homogeneous finite strains.

\subsection{Necking of a steel circular bar}

In this example we show that the general computational elastoplasticity formulation presented above in terms of finite elastic logarithmic strains and their work-conjugate generalized Kirchhoff stresses in full Lagrangian symmetric space description give the same results than other classical formulations under isotropic elasto-plasticity conditions. We analyze the necking of a metallic circular bar, which undergoes finite plastic strains combined with moderate elastic strains.

We use a Voce-type non-linear isotropic hardening function [70]

$$
k=k_{0}+\bar{H} E_{p}+\left(k_{\infty}-k_{0}\right)\left(1-e^{-\delta E_{p}}\right)
$$



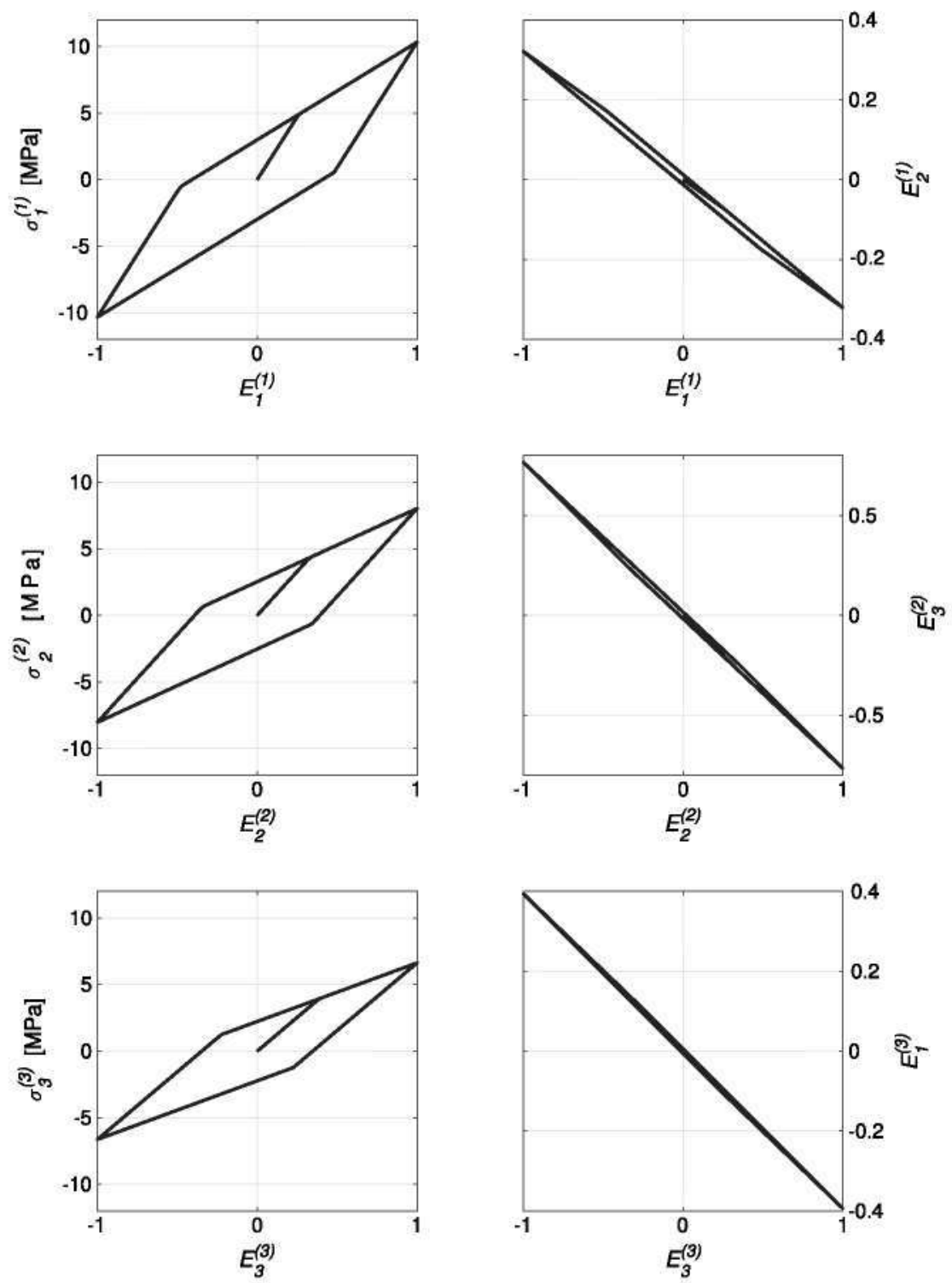

Fig. 8. Uniaxial loading-unloading tests over orthotropy preferred directions. We represent by $\sigma_{i}^{(i)}$ the uniaxial Cauchy stress component $i$ in the test performed in axis $(i)$ and by $E_{j}^{(i)}$ the transverse logarithmic strain component $j$ in the test performed in axis $(i)$. Case with kinematic hardening only, i.e. with $k^{\prime}=0$ and $2 \mu_{i}^{k i n}>0, i=1,2,3$.

where here $E_{p}$ is the effective logarithmic plastic strain, $\bar{H}$ is the linear hardening modulus, $k_{0}$ is the reference yield stress and $k_{\infty}$ and $\delta$ are saturation law material parameters. For our model, we recognize $E_{p} \equiv \gamma$ in the previous law, recall Eq. (149). The isotropic elastic material parameters are $Y_{e}=206.9 \mathrm{GPa}$ and $v_{e}=0.29$, from which we obtain - note that $\mu_{k i n}=0$ because only isotropic hardening is being considered

$$
2 \mu_{e}=2 \mu_{i n t}=\frac{Y_{e}}{1+v_{e}}=160.4 G P a \quad \text { and } \quad \kappa=\frac{Y_{e}}{3\left(1-2 v_{e}\right)}=164.2 \mathrm{GPa} .
$$

The isotropic plastic parameters are

$$
k_{0}=0.45 \mathrm{GPa}, \quad \bar{H}=0.12924 \mathrm{GPa}, \quad k_{\infty}=0.715 \mathrm{GPa} \quad \text { and } \quad \delta=16.93 .
$$

We use the von Mises yield criterion, i.e. we take $\overline{\mathbb{N}}=\mathbb{I}$ in Eq. (133). 

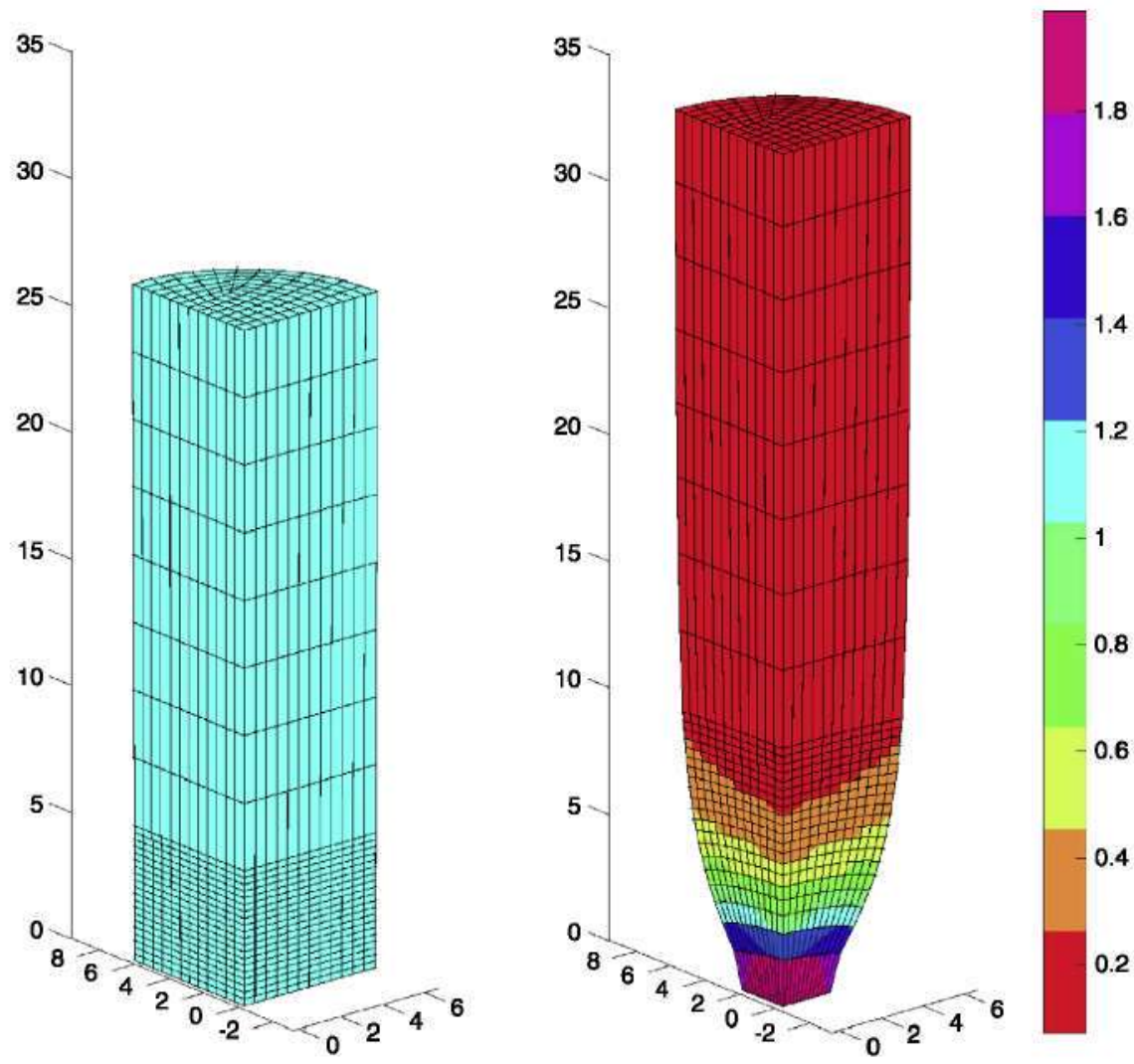

Fig. 9. Circular bar. Left: Finite element undeformed three-dimensional mesh being considered (eighth of the complete geometry). Isotropic elastoplasticity conditions. Bar total length $=53.34 \mathrm{~mm}$; Bar radius $=6.4135 \mathrm{~mm}$. Right: Deformed configuration and distribution of $\gamma$ for a total length increment of $14 \mathrm{~mm}$. Unaveraged results at nodes.

The bar is stretched along its major axis by prescribing a displacement of $7 \mathrm{~mm}$ at each end to give a total final displacement of $14 \mathrm{~mm}$, see Fig. 9. Only an eighth of the total three-dimensional mesh is considered by symmetry conditions. In order to prevent mesh locking during plastic flow, fully integrated $(3 \times 3 \times 3$ Gauss integration) $27 / 4$, $u / p$ mixed finite elements are used. A standard Newton-Raphson scheme, without line searches, is employed for the incremental solution obtained by our in-house finite element code Dulcinea.

In Fig. 9 we also show the deformed mesh and the distribution of equivalent logarithmic plastic strain (i.e. the consistency parameter $\gamma$ ) for the maximum displacement being applied. The non-homogeneous solution obtained using the present formulation is almost indistinguishable from the solution obtained by Caminero et al. in Ref. [45], which was obtained using the same mesh and the same finite element code. In Ref. [45] the non-homogeneous solution was compared with the response given by the Eterovic-Bathe isotropic model [21], giving also the same results.

In Fig. 10 we show the load-deflection results obtained from the simulation using our model, which are in agreement with the results obtained from other computational elastoplasticity models in the literature, namely Refs. [38,71,72,73,45],

\subsection{Extension of a soft rectangular plate with a hole}

We perform in this example finite element elastoplasticity computations with the following noteworthy characteristics: both elastic and plastic non-homogeneous finite strains are present, fully orthotropic elastoplastic behavior is considered and combined isotropic-kinematic hardening effects are included. These are, in fact, the most complete simulations that may be carried out with the present anisotropic finite strain elastoplasticity model which, in turn, show its excellent computational performance. 


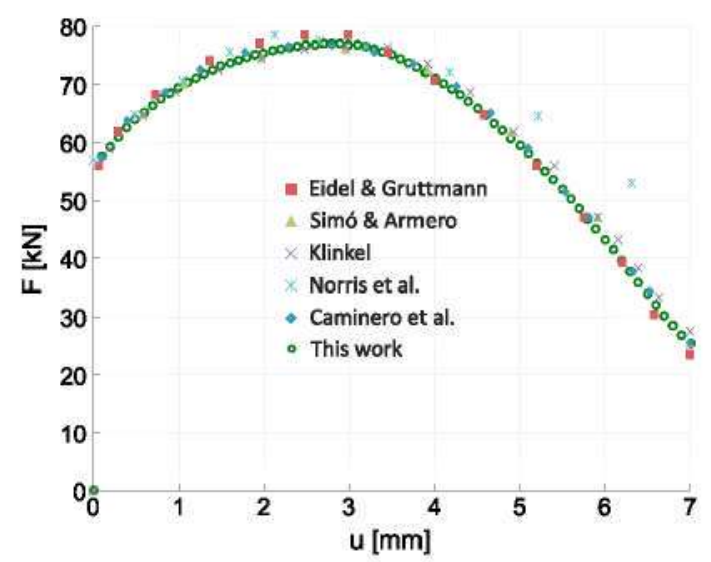

Fig. 10. Necking of a circular bar. Different simulation results and experimental data. Load-displacement curve: applied force F [kN] versus applied displacement $\mathrm{u}[\mathrm{mm}]$.

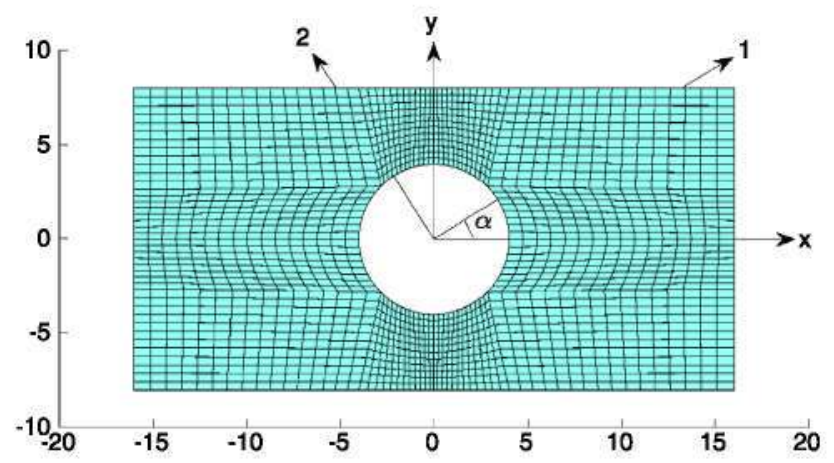

Fig. 11. Plate with a circular hole. Finite element undeformed bidimensional mesh under a plane strain condition. Length $=32 \mathrm{~mm}$; Height $=16 \mathrm{~mm}$; Hole Diameter $=8 \mathrm{~mm}$. The angle $\alpha$ defines the orientation of the preferred material axis 1 (i.e. direction $\boldsymbol{a}_{1}$ ) with respect to the horizontal axis $x$.

We show in Fig. 11 the undeformed mesh used for the simulations. In this case, bidimensional fully integrated (3 $\times 3$ Gauss integration) $9 / 3, u / p$ mixed finite elements are used. The plate is stretched in $x$-direction up to a total elongation of $l=38.4 \mathrm{~mm}$, which corresponds to an average deformation of $20 \%$. We assume perfectly lubricated grips at both ends and a plane strain condition.

In the first set of simulations addressed in this example we consider a nearly-incompressible elastoplastic response including only kinematic hardening effects. We have used the following model parameters for the deviatoric energy functions of Eq. (128) and the Hill yield tensor of Eq. (133)

$$
\begin{aligned}
& \mu_{1}^{i n t}=8 \mathrm{MPa}, \quad \mu_{2}^{i n t}=5 \mathrm{MPa}, \quad \mu_{3}^{i n t}=2 \mathrm{MPa}, \quad \mu_{4}^{i n t}=4 \mathrm{MPa} \\
& \mu_{1}^{k i n}=3 \mathrm{MPa}, \quad \mu_{2}^{k i n}=2 \mathrm{MPa}, \quad \mu_{3}^{k i n}=1 \mathrm{MPa}, \quad \mu_{4}^{k i n}=1.5 \mathrm{MPa}
\end{aligned}
$$

and

$$
k=k_{0}=4.4 \mathrm{MPa}, \quad(\overline{\mathbb{N}})_{22}=2.125, \quad(\overline{\mathbb{N}})_{33}=3.25, \quad(\overline{\mathbb{N}})_{44}=1
$$

where we have used Voigt index contractions $(11) \rightarrow(1),(22) \rightarrow(2),(33) \rightarrow(3)$ and (12) $\rightarrow$ (4). The penalty-type volumetric strain energy employed in this case is

$$
\mathcal{U}(J)=\kappa(1+J(\ln J-1))
$$

with $\kappa=10^{4} \mathrm{MPa}$. 
Table 2

Cases with kinematic hardening only (Fig. 12). Residual force and energy during typical hyperelastic and elastoplastic steps.

\begin{tabular}{lll}
\hline Step/Iteration & Force & Energy \\
\hline $4 / 1$ & $1.000 \mathrm{E}+00$ & $1.000 \mathrm{E}+00$ \\
$4 / 2$ & $1.753 \mathrm{E}-03$ & $3.929 \mathrm{E}-06$ \\
$4 / 3$ & $1.584 \mathrm{E}-06$ & $1.597 \mathrm{E}-12$ \\
$4 / 4$ & $3.613 \mathrm{E}-12$ & $2.237 \mathrm{E}-25$ \\
\hline $15 / 1$ & $1.000 \mathrm{E}+00$ & $1.000 \mathrm{E}+00$ \\
$15 / 2$ & $1.326 \mathrm{E}-02$ & $1.822 \mathrm{E}-04$ \\
$15 / 3$ & $1.218 \mathrm{E}-03$ & $2.409 \mathrm{E}-07$ \\
$15 / 4$ & $4.342 \mathrm{E}-06$ & $2.507 \mathrm{E}-12$ \\
$15 / 5$ & $1.355 \mathrm{E}-11$ & $2.196 \mathrm{E}-22$ \\
\hline
\end{tabular}

We show the deformed meshes for maximum load and the consistency parameter band plots (i.e. accumulated plastic logarithmic deformation) for four different orientations of the preferred material axes in Fig. 12, with the angle $\alpha$ defining the orientation of the preferred material axis 1 with respect to the horizontal axis $x$, see Fig. 11 . The angular distortion experienced by the plate is consistent with the expected response in all the cases, as explained in Ref. [49], Appendix 3. We can observe that a necking effect is being initiated in the central passing area, which is more pronounced for the cases with orientations $\alpha=0^{\circ}$ and $\alpha=15^{\circ}$. These plastic strains are accompanied by elastic strains of the same order of magnitude in that zone. Indeed, the total displacement load has been applied incrementally in 16 steps in all the cases $(0.4 \mathrm{~mm} / \mathrm{step})$ and the first $4-5$ steps (depending on the case) have resulted purely hyperelastic. Remarkably, very similar (excellent) rates of convergence for residual force and energy have been attained during hyperelastic steps (4 iterations) and elastoplastic steps (5 iterations), see Table 2.

In the second set of simulations performed in this example we add an isotropic hardening effect to the material model defined above. We consider the following isotropic hardening linear in $\gamma$

$$
k=k_{0}+\bar{H} \gamma
$$

with $k_{0}=4.4 \mathrm{MPa}$ and also $\bar{H}=4.4 \mathrm{MPa}$ (i.e. $k$ is doubled for $\gamma=1$ ). The results obtained in this mixed hardening cases are shown in Fig. 13 for the different material axes orientations, where we can observe that the accumulated plastic deformation is reduced by half with respect to the respective maximum values in Fig. 12. Necking effects have been reduced in all the cases. Accordingly, the elastic strains (and associated stresses) are higher in these cases as well. Again, asymptotically quadratic rates of convergence have been attained during the elastoplastic loading in very few steps, see Table 3 , which proves the high computational efficiency of the fully symmetric finite element formulation for multiplicative anisotropic elastoplasticity presented in this work.

These orthotropic non-homogeneous finite element models have been computed using one processor of a 2011 Windows-PC with our in-house finite element code Dulcinea running as a 32-bit fortran90 Pentium application. Under these conditions, each computational simulation addressed in this example needed about 10 min (elapsed time) in average. The inclusion of the isotropic hardening effect in the second set of simulations did not increase the simulation time significantly with respect to the first set of simulations.

\section{Conclusions}

In this paper we present a novel computational framework for anisotropic elastoplasticity suitable for finite element implementation. The formulation is valid for multiplicative elastoplasticity employing large strain, fully nonlinear anisotropic hyperelasticity. The formulation is motivated from a one-dimensional rheological model and accommodates the phenomenological effects of isotropic, kinematic or combined hardening.

The most relevant contribution of the new nonlinear algorithmic framework, which uses the multiplicative decomposition, is that it does not explicitly employ plastic strains or plastic metric measures. Instead, an elastic correction is used which results in an additive six-dimensional update from a backward-Euler rule parallel to that of 


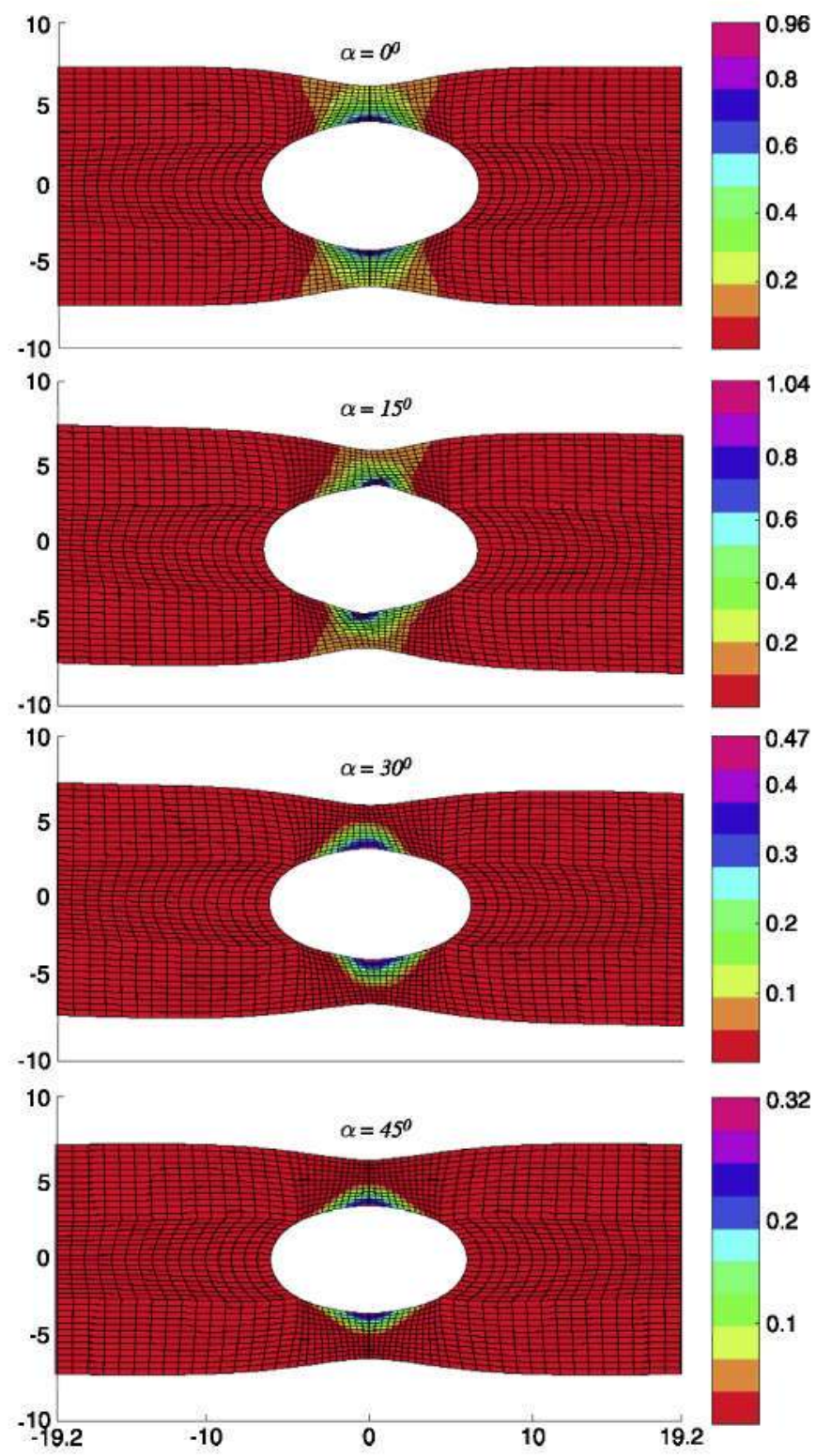

Fig. 12. Case with kinematic hardening. Deformed configurations and distributions of $\gamma$ for $\alpha=0^{\circ}, \alpha=15^{\circ}, \alpha=30^{\circ}$ and $\alpha=45^{\circ}$. Unaveraged results at nodes.

Table 3

Cases with combined kinematic and isotropic hardening (Fig. 13). Residual force and energy during a typical elastoplastic step.

\begin{tabular}{lll}
\hline Step/Iteration & Force & Energy \\
\hline $15 / 1$ & $1.000 \mathrm{E}+00$ & $1.000 \mathrm{E}+00$ \\
$15 / 2$ & $2.620 \mathrm{E}-03$ & $1.822 \mathrm{E}-05$ \\
$15 / 3$ & $1.219 \mathrm{E}-03$ & $2.409 \mathrm{E}-06$ \\
$15 / 4$ & $1.806 \mathrm{E}-05$ & $2.507 \mathrm{E}-10$ \\
$15 / 5$ & $6.958 \mathrm{E}-09$ & $2.196 \mathrm{E}-18$ \\
\hline
\end{tabular}

the infinitesimal setting, without explicitly employing exponential mappings. Furthermore, the Mandel stress tensor plays no role in the present formulation and we do not need to establish any assumption in the plastic spin to integrate 


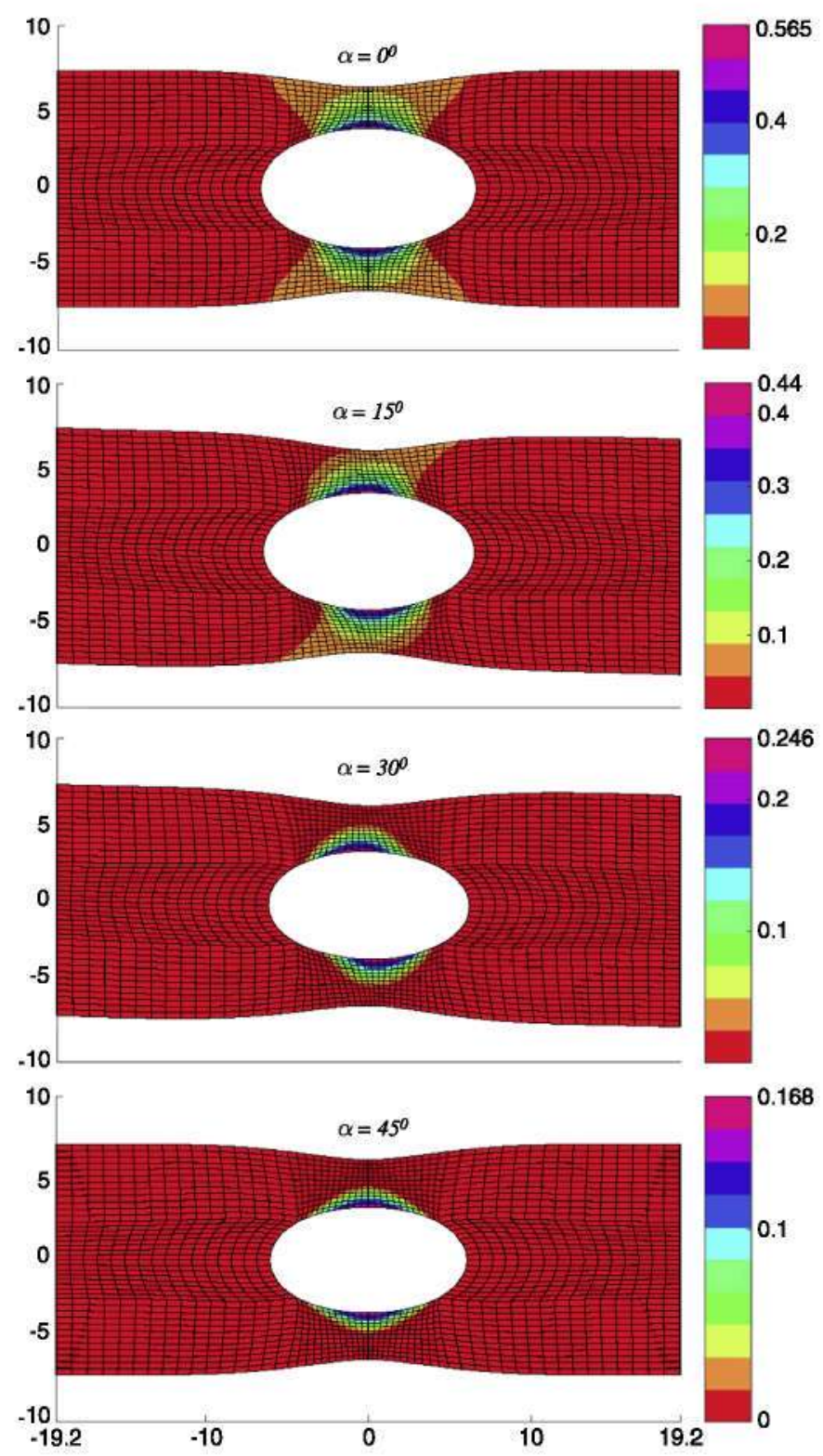

Fig. 13. Case with combined isotropic-kinematic hardening. Deformed configurations and distributions of $\gamma$ for $\alpha=0^{\circ}, \alpha=15^{\circ}, \alpha=30^{\circ}$ and $\alpha=45^{\circ}$. Unaveraged results at nodes.

the symmetric flow. If desired, an evolution equation for the plastic spin may be established in a completely uncoupled procedure.

In the numerical examples we show that some previously proposed formulations may be recovered as particular cases and that the numerical efficiency of the algorithm is excellent, obtaining convergence rates close to those of the hyperelastic model.

For simplicity, in these examples we have used stored energies which are quadratic in logarithmic strains. However, the general formulation is not restricted to linearity in those strains and can also immediately accommodate nonlinear hardening effects. Although the present formulation can properly simulate the Bauschinger effect, our hardening formulation does not have an explicit backstress. Therefore, the implementation of the Armstrong and Frederick rule and the comparison of the resulting multiaxial behavior remains a matter for further research. 


\section{Acknowledgments}

Partial financial support for this work has been given by grant DPI2015-69801-R from the Dirección General de Proyectos de Investigación of the Ministerio de Economía y Competitividad of Spain. F.J. Montáns also acknowledges the support of the Department of Mechanical and Aerospace Engineering of University of Florida during the sabbatical period in which this paper was finally completed and Ministerio de Educación, Cultura y Deporte of Spain for the financial support for that stay under grant PRX15/00065.

\section{References}

[1] K.J. Bathe, Finite Element Procedures, second ed., K.J Bathe, Watertown, 2014.

[2] M. Kojić, K.J. Bathe, Inelastic Analysis of Solids and Structures, Springer, Berlin, 2005.

[3] D. Raabe, F. Roters, F. Barlat, L. Chen (Eds.), Continuum Scale Simulation of Engineering Materials: Fundamentals-Microstructures-Process Applications, John Wiley \& Sons, 2006.

[4] D. Banabic, Sheet Metal Forming Processes: Constitutive Modeling and Numerical Simulation, Springer Science \& Business Media, 2010.

[5] M.L. Wilkins, Calculation of elastic-plastic flow, in: B. Alder, S. Fernback, M. Rotenberg (Eds.), in: Methods of Computational Physics, vol. 3, Academic Press, New York, 1964.

[6] G. Maenchen, S. Sacks, The tensor code, in: B. Alder, S. Fernback, M. Rotenberg (Eds.), in: Methods of Computational Physics, vol. 3, Academic Press, New York, 1964.

[7] R.D. Krieg, S.W. Key, Implementation of a time dependent plasticity theory into structural computer programs, in: J.A. Stricklin, K.J. Saczlski (Eds.), Constitutive Equations in Viscoplasticity: Computational and Engineering Aspects, AMD-20, ASME, New York, 1976.

[8] J.C. Simo, Numerical analysis and simulation of plasticity, in: Handbook of Numerical Analysis, 1998, pp. 183-499.

[9] J. Lubliner, Plasticity Theory, Courier Corporation, 2008.

[10] A.V. Shutov, J. Ihlemann, Analysis of some basic approaches to finite strain elasto-plasticity in view of reference change, Int. J. Plast, 63 (2014) 183-197.

[11] J.C. Simo, Algorithms for static and dynamic multiplicative plasticity that preserve the classical return mapping schemes of the infinitesimal theory, Comput. Methods Appl. Mech. Engrg. 99 (1) (1992) 61-112.

[12] H.D. Hibbitt, P.V. Marcal, J.R. Rice, A finite element formulation for problems of large strain and large displacement, Int. J. Solids Struct. $6(8)(1970) 1069-1086$

[13] R.M. McMeeking, J.R. Rice, Finite-element formulations for problems of large elastic-plastic deformation, Int. J. Solids Struct, 11 (5) (1975) 601-616.

[14] S.W. Key, R.D. Krieg, On the numerical implementation of inelastic time dependent and time independent, finite strain constitutive equations in structural mechanics, Comput. Methods Appl. Mech. Engrg. 33 (1) (1982) 439-452.

[15] L.M. Taylor, E.B. Becker, Some computational aspects of large deformation, rate-dependent plasticity problems, Comput. Methods Appl. Mech. Engrg, 41 (3) (1983) 251-277.

[16] J.C. Simó, K.S. Pister, Remarks on rate constitutive equations for finite deformation problems: computational implications, Comput. Methods Appl. Mech. Engrg. 46 (2) (1984) 201-215.

[17] M. Kojić, K.J. Bathe, Studies of finite element procedures - Stress solution of a closed elastic strain path with stretching and shearing using the updated Lagrangian Jaumann formulation, Comput. Struct. 26 (1) (1987) 175-179.

[18] J,C. Simo, A framework for finite strain elastoplasticity based on maximum plastic dissipation and the multiplicative decomposition: Part I. Continuum formulation, Comput. Methods Appl. Mech. Engrg. 66 (2) (1988) 199-219.

[19] A.E. Green, P.M. Naghdi, Some remarks on elastic-plastic deformation at finite strain, Internat. J. Engrg. Sci. 9 (12) (1971) 1219 -1229.

[20] G. Weber, L. Anand, Finite deformation constitutive equations and a time integration procedure for isotropic, hyperelastic-viscoplastic solids, Comput. Methods Appl. Mech. Engrg. 79 (2) (1990) 173-202.

[21] A.L. Eterovic, K.J. Bathe, A hyperelastic-based large strain elasto-plastic constitutive formulation with combined isotropic-kinematic hardening using the logarithmic stress and strain measures, Internat. J, Numer. Methods Engrg. 30 (6) (1990) 1099-1114.

[22] D. Peric, D.R.J. Owen, M.E. Honnor, A model for finite strain elasto-plasticity based on logarithmic strains: Computational issues, Comput. Methods Appl. Mech. Engrg. 94 (1) (1992) 35-61.

[23] A. Cuitiño, M. Ortiz, A material-independent method for extending stress update algorithms from small-strain plasticity to finite plasticity with multiplicative kinematics, Eng, Comput. 9 (4) (1992) 437-451.

[24] C. Eckart, The thermodynamics of irreversible processes. IV. The theory of elasticity and anelasticity, Phys. Rev. 73 (4) (1948) $373-382$.

[25] J.F. Besseling, A thermodynamic approach to rheology, in: H. Parkus, L.I. Sedov (Eds.), Irreversible Aspects of Continuum Mechanics and Transfer of Physical Characteristics in Moving Fluids, Springer, Vienna, 1968, pp. 16-53.

[26] A.I. Leonov, Nonequilibrium thermodynamics and rheology of viscoelastic polymer media, Rheol. Acta 15 (2) (1976) 85-98.

[27] M.B. Rubin, O. Vorobiev, E. Vitali, A thermomechanical anisotropic model for shock loading of elastic-plastic and elastic-viscoplastic materials with application to jointed rock, Comput. Mech. (2016) 1-22.

[28] A.E. Green, P.M. Naghdi, A general theory of an elastic-plastic continuum, Arch. Ration. Mech. Anal. 18 (4) (1965) $251-281$.

[29] J.C. Simó, M. Ortiz, A unified approach to finite deformation elastoplastic analysis based on the use of hyperelastic constitutive equations, Comput. Methods Appl. Mech. Engrg. 49 (2) (1985) 221-245.

[30] C. Miehe, A formulation of finite elastoplasticity based on dual co-and contra-variant eigenvector triads normalized with respect to a plastic metric, Comput. Methods Appl. Mech. Engrg. 159 (3) (1998) 223-260. 
[31] P. Papadopoulos, J. Lu, A general framework for the numerical solution of problems in finite elasto-plasticity, Comput. Methods Appl. Mech. Engrg. 159 (1) (1998) 1-18.

[32] I. Schmidt, Some comments on formulations of anisotropic plasticity, Comput. Mater. Sci. 32 (3) (2005) $518-523$.

[33] M. Itskov, On the application of the additive decomposition of generalized strain measures in large strain plasticity, Mech. Res. Commun. 31 (5) (2004) 507-517.

[34] P. Neff, I.D. Ghiba, Loss of ellipticity for non-coaxial plastic deformations in additive logarithmic finite strain plasticity, Int. J. Non-Linear Mech. 81 (2016) 122-128.

[35] E.H. Lee, Elastic-plastic deformations at finite strains, J. Appl. Mech. 36 (1969) 1-6.

[36] J. Mandel, Thermodynamics and plasticity, in: J.J. Delgado Domingers, N.R. Nina, J.H. Whitelaw (Eds.), Foundations of Continuum Thermodynamics, Macmillan, London, 1974, pp. 283-304.

[37] A. Lion, Constitutive modeling in finite thermoviscoplasticity: a physical approach based on nonlinear rheological models, Int. J. Plast. 16 (5) (2000) $469-494$.

[38] B. Eidel, F. Gruttmann, Elastoplastic orthotropy at finite strains: multiplicative formulation and numerical implementation, Comput. Mater. Sci. 28 (3) (2003) 732-742.

[39] S. Chatti, A. Dogui, P. Dubujet, F. Sidoroff, An objective incremental formulation for the solution of anisotropic elastoplastic problems at finite strain, Commun. Numer. Methods. Eng. 17 (12) (2001) 845-862.

[40] C.S. Han, K. Chung, R.H. Wagoner, S.I. Oh, A multiplicative finite elasto-plastic formulation with anisotropic yield functions, Int. J. Plast. 19 (2) (2003) 197-211.

[41] A. Menzel, M. Ekh, K. Runesson, P. Steinmann, A framework for multiplicative elastoplasticity with kinematic hardening coupled to anisotropic damage, Int. J. Plast. 21 (3) (2005) 397-434.

[42] C. Sansour, I. Karłaj, J. Sorić, A formulation of anisotropic continuum elastoplasticity at finite strains. Part I: Modeling, Int. J. Plast, 22 (12) (2006) $2346-2365$.

[43] F.J. Montáns, K.J. Bathe, Towards a model for large strain anisotropic elasto-plasticity, in: E. Oñate, R. Owen (Eds.), Computational Plasticity, Springer, Netherlands, 2007, pp. 13-36.

[44] I.N. Vladimirov, M.P. Pietryga, S. Reese, Anisotropic finite elastoplasticity with nonlinear kinematic and isotropic hardening and application to sheet metal forming, Int. J. Plast. 26 (5) (2010) 659-687.

[45] M.Á. Caminero, F.J. Montáns, K.J. Bathe, Modeling large strain anisotropic elasto-plasticity with logarithmic strain and stress measures. Comput. Struct. 89 (11) (2011) 826-843.

[46] G.I. Taylor, Analysis of plastic strain in a cubic crystal, in: J.M. Lessels (Ed.), Stephen Timoshenko 60th Anniversary Volume, Macmillan, New York, 1938 ,

[47] J.R. Rice, Inelastic constitutive relations for solids: an internal-variable theory and its application to metal plasticity, J. Mech. Phys. Solids 19 (6) (1971) $433-455$.

[48] A.V. Shutov, Efficient implicit integration for finite-strain viscoplasticity with a nested multiplicative split, Comput. Methods Appl. Mech. Engrg. 306 (2016) 151-174.

[49] M. Latorre, F.J. Montáns, A new class of plastic flow evolution equations for anisotropic multiplicative elastoplasticity based on the notion of a corrector elastic strain rate, 2016. ArXiv:1701.00095vl [cond-mat.soft].

[50] O.T. Bruhns, The multiplicative decomposition of the deformation gradient in plasticity — origin and limitations, in: H. Altenbach, T. Matsuda. D. Okumura (Eds.), From Creep Damage Mechanics to Homogenization Methods, in: Advanced Structured Materials, vol. 64, Springer International Publishing, 2015, pp. 37-66.

[51] J.C Simo, C. Miehe, Associative coupled thermoplasticity at finite strains: formulation, numerical analysis and implementation, Comput. Methods Appl. Mech. Engrg. 98 (1) (1992) 41-104.

[52] M. Latorre, F.J. Montáns, Stress and strain mapping tensors and general work-conjugacy in large strain continuum mechanics, Appl. Math. Model. 40 (5) (2016) 3938-3950.

[53] J. Mandel, Plasticité Classique et Viscoplasticité. Course held at the Department of Mechanics of Solids, Springer, New York, 1972.

[54] J. Bonet, R.D. Wood, Nonlinear Continuum Mechanics for Finite Element Analysis, second ed., Cambridge University Press, 2008.

[55] J. Lubliner, Normality rules in large-deformation plasticity, Mech. Mater. 5 (1) (1986) 29-34.

[56] M. Latorre, F.J. Montáns, On the interpretation of the logarithmic strain tensor in an arbitrary system of representation, Int. J. Solids Struct. 51 (7) (2014) 1507-1515

[57] J.H. Argyris, J.S. Doltsinis, P.M. Pimenta, H. Wüstenberg, Thermomechanical response of solids at high strains - natural approach, Comput. Methods Appl. Mech. Engrg. 32 (1-3) (1982) 3-57.

[58] F.J. Ulm, O. Coussy, Mechanies and Durability of Solids. Volume I: Solid Mechanics, Prentice Hall, Harlow, United Kingdom, 2003.

[59] I.N, Vladimirov, M.P. Pietryga, S. Reese, On the modeling of non-linear kinematic hardening at finite strains with application to springbackComparison of time integration algorithms, Internat. J. Numer. Methods Engrg. 75 (1) (2008) 1-28.

[60] M. Latorre, F.J. Montáns, Anisotropic finite strain viscoelasticity based on the Sidoroff multiplicative decomposition and logarithmic strains, Comput. Mech. 56 (3) (2015) 503-531.

[61] W. Prager, Der Einflußder Verformung auf die Fließbedingung zähplastischer Körper, ZAMM Z. Angew. Math. Mech. 15 (1-2) (1935) 76-80.

[62] M. Latorre, F.J. Montáns, Fully anisotropic finite strain viscoelasticity based on a reverse multiplicative decomposition and logarithmic strains. Comput. Struct. 163 (2016) 56-70.

[63] J.C. Simó, T.J.R. Hughes, Computational Inelasticity, Springer, New York, 1998.

[64] F.J. Montâns, I.M. Benítez, M.A. Caminero, A large strain anisotropic elastoplastic continuum theory for nonlinear kinematic hardening and texture evolution, Mech. Res. Commun. 43 (2012) $50-56$.

[65] D.N. Kim, F.J. Montáns, K.J. Bathe, Insight into a model for large strain anisotropic elasto-plasticity, Comput. Mech. 44 (5) (2009) 651-668. 
[66] M. Miñano, M.Á. Caminero, F.J. Montáns, On the numerical implementation of the Closest Point Projection algorithm in anisotropic elastoplasticity with nonlinear mixed hardening, Finite Elem. Anal. Des. 121 (2016) 1-17.

[67] M. Itskov, N. Aksel, Elastic constants and their admissible values for incompressible and slightly compressible anisotropic materials, Acta Mech. 157 (2002) 81-96.

[68] L. Anand, On H. Hencky's approximate strain-energy function for moderate deformations, J. Appl. Mech. 46 (1) (1979) $78-82$.

[69] L. Anand, Moderate deformations in extension-torsion of incompressible isotropic elastic materials, J. Mech. Phys. Solids 34 (3) (1986) 293-304.

[70] E. Voce, A practical strain-hardening function, Metallurgica 51 (1955) 219-226.

[71] J.C. Simo, F. Armero, Geometrically non-linear enhanced strain mixed methods and the method of incompatible modes, Internat. J. Numer. Methods Engrg. 33 (1992) 1413-1449.

[72] S. Klinkel, Theorie und Numerik eines Volumen-Schalen-Elementes bei finiten elastischen und plastischen Verzerrungen (Ph.D. thesis), University of Karlsruhe, 2000.

[73] D.M. Norris, B. Moran, J.K. Scudder, D.F. Quiñones, A computer simulation of the tension test, J. Mech. Phys. Solids 26 (1978) 1-19. 